\title{
SUPPORTING INFORMATION \\ Combined NMR and computational study of cysteine oxidation during nucleation of metallic clusters in biological systems
}

Barbara Pem ${ }^{1}$, Mateja Toma², Valerije Vrček², Ivana Vinković Vrček ${ }^{1 *}$

${ }^{1}$ Institute for Medical Research and Occupational Health, Ksaverska cesta 2, Zagreb, Croatia

${ }^{2}$ Faculty of Pharmacy and Biochemistry, University of Zagreb, Ante Kovačića 1, Zagreb, Croatia

Contents:

1. Supplemental information on nanoparticle (NP) preparation and characterization

1.1. NP preparation conditions and mechanism

1.2. UV/VIS spectroscopy characterization

2. NMR spectra

3. Additional computational information - Images and energy tables

4. $X Y Z$ coordinates of all structures from theoretical calculations 


\section{Supplemental information on nanoparticles preparation and characterization}

\subsection{NP preparation conditions and mechanism}

The optimization of reaction conditions was necessary to ensure the preparation of small, spherical and stable NPs that would not precipitate in the duration of the study. The optimization method was described in our previous publication (Pem, B.; Pongrac, I. M.; Ulm, L.; Pavičić, l.; Vrček, V.; Domazet Jurašin, D.; Ljubojević, M.; Krivohlavek, A.; Vinković Vrček, I. Toxicity and Safety Study of Silver and Gold Nanoparticles Functionalized with Cysteine and Glutathione. Beilstein J. Nanotechnol. 2019, 10, 1802-1817), and here briefly summarized. The molar ratios of reagents were varied, and the resulting NPs were monitored visually (to check for precipitation) and by DLS (size measurements). Also, two approaches were tried considering the order of addition of reagents: one where $\mathrm{NaBH}_{4}$ would be added to the metallic salt solution first, followed by cysteine, and another where cysteine would be added before $\mathrm{NaBH}_{4}$. As seen from Table $\mathrm{S} 1$, the 10 -fold molar excess of $\mathrm{NaBH}_{4}$ was beneficial for successful preparation, which was also confirmed by literature reports (Song, K. C.; Lee, S. M.; Park, T. S.; Lee, B. S. Preparation of Colloidal Silver Nanoparticles by Chemical Reduction Method. Korean J. Chem. Eng. 2009, 26, 153-155.). The concentrations of cysteine below $1 \mathrm{mM}$, while favorable, did not allow for NMR measurements due to detection limits. Therefore, the molar ratio of metal salt : $\mathrm{NaBH}_{4}:$ cysteine $=1: 10: 1$ was ultimately selected. Though excellent stability and favorable size distribution were achieved with metal/cysteine concentrations of $5.6 \mathrm{mM}$ (for $\mathrm{Ag}$ ) or $3 \mathrm{mM}$ (for $\mathrm{Au}$ ), in order increase the quality of the NMR spectra all the concentrations were raised to $10 \mathrm{mM}$. The NP characteristics remained satisfactory and there were no significant changes in chemical shifts or multiplicity of NMR signals. 
Table S1. Conditions for the preparation of AgNPs and AuNPs by reduction of metal salts $\mathrm{AgNO}_{3}$ and $\mathrm{HauCl}_{4}$, respectively) with $\mathrm{NaBH}_{4}$ in the presence of cysteine (CYS), along with the visual properties and size of the final NPs. Data marked with asterisks $\left({ }^{*}\right)$ were taken from our previous study (Pem, B.; Pongrac, I. M.; Ulm, L.; Pavičić, I.; Vrček, V.; Domazet Jurašin, D.; Ljubojević, M.; Krivohlavek, A.; Vinković Vrček, I. Toxicity and Safety Study of Silver and Gold Nanoparticles Functionalized with Cysteine and Glutathione. Beilstein J. Nanotechnol. 2019, 10, 1802-1817).

\begin{tabular}{|c|c|c|c|c|c|}
\hline Metal & $\begin{array}{l}\text { Metal } \\
\text { conc. } \\
{[\mathrm{mm}]}\end{array}$ & Metal : $\mathrm{NaBH}_{4}$ : coating & $\begin{array}{l}\text { Last } \\
\text { added } \\
\text { reactant }\end{array}$ & Visual characteristics & $\begin{array}{l}\text { Size }[\mathrm{nm}] \\
\text { (\% volume) }\end{array}$ \\
\hline $\mathrm{Ag}$ & 1 & $1: 2: 0.2$ & CYS & * Brown, stable & $* 3.0 \pm 0.3(100 \%)$ \\
\hline $\mathrm{Ag}$ & 1 & $1: 2: 0.2$ & $\mathrm{NaBH}_{4}$ & * Dark brown, stable & $* 15.5 \pm 2.0(100 \%)$ \\
\hline $\mathrm{Ag}$ & 1 & $1: 2: 0.5$ & CYS & * Brown-gray, stable & n.a. \\
\hline $\mathrm{Ag}$ & 1 & $1: 2: 0.5$ & $\mathrm{NaBH}_{4}$ & * Dark gray, unstable & n.a. \\
\hline $\mathrm{Ag}$ & 1 & $1: 10: 0.2$ & CYS & * Brown, unstable & $* 3.3 \pm 0.6(100 \%)$ \\
\hline $\mathrm{Ag}$ & 1 & $1: 10: 0.2$ & $\mathrm{NaBH}_{4}$ & * Brown, unstable & $\begin{array}{l}* 1.7 \pm 0.8(98 \%) \\
15.2 \pm 1.7(2 \%)\end{array}$ \\
\hline $\mathrm{Ag}$ & 5.6 & $1: 10: 1$ & $\mathrm{NaBH}_{4}$ & * Brown, stable & $\begin{array}{l}* 8.0 \pm 0.9(99 \%) \\
309.7 \pm 98.8(<1 \%)\end{array}$ \\
\hline $\mathrm{Ag}$ & 10 & $1: 10: 1$ & $\mathrm{NaBH}_{4}$ & Brown, stable & $\begin{array}{l}14.4 \pm 4.2(46 \%) \\
41.7 \pm 12.1(47 \%) \\
279.9 \pm 99.4(7 \%)\end{array}$ \\
\hline $\mathrm{Au}$ & 1 & $1: 2: 0.2$ & CYS & * Blue-gray, unstable & n.a. \\
\hline $\mathrm{Au}$ & 1 & $1: 2: 0.2$ & $\mathrm{NaBH}_{4}$ & * Unstable & n.a. \\
\hline $\mathrm{Au}$ & 1 & $1: 2: 1$ & CYS & * Purple, unstable & n.a. \\
\hline $\mathrm{Au}$ & 1 & $1: 2: 1$ & $\mathrm{NaBH}_{4}$ & * Gray, unstable & n.a. \\
\hline $\mathrm{Au}$ & 1 & $1: 10: 0.2$ & $\mathrm{NaBH}_{4}$ & * Purple, stable & $\begin{array}{l}* 10.7 \pm 3.7(30 \%) \\
48.5 \pm 5.8(70 \%)\end{array}$ \\
\hline $\mathrm{Au}$ & 3 & $1: 10: 1$ & $\mathrm{NaBH}_{4}$ & * Purple, stable & $\begin{array}{l}* 24.2 \pm 3.4(54 \%) \\
80.0 \pm 2.1(10 \%) \\
219.7 \pm 48.9(35 \%)\end{array}$ \\
\hline $\mathrm{Au}$ & 10 & $1: 10: 1$ & $\mathrm{NaBH}_{4}$ & $\begin{array}{l}\text { Dark purple, but } \\
\text { precipitate }\end{array}$ & $\begin{array}{l}42.2 \pm 4.4(74 \%) \\
341,1 \pm 64.5(26 \%)\end{array}$ \\
\hline
\end{tabular}




\subsection{UV/VIS spectroscopy characterization}

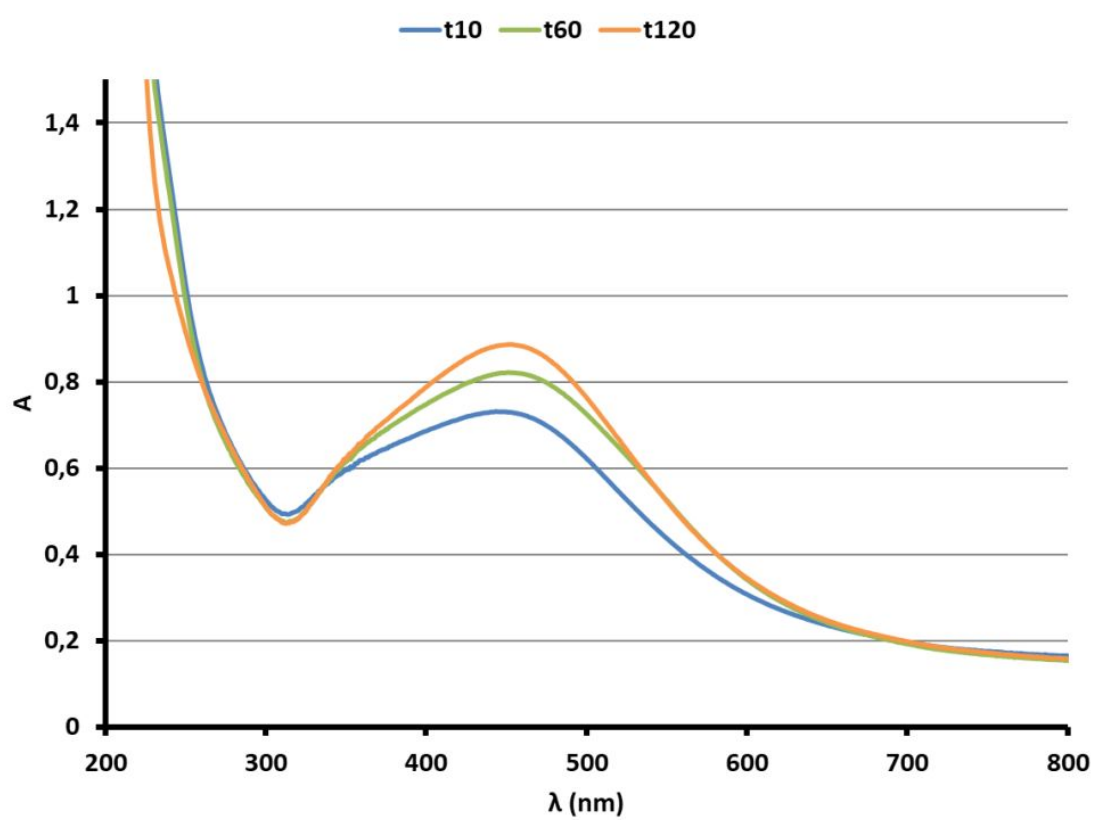

Figure S1. The UV/VIS spectra of the reaction mixture containing $\mathrm{AgNO}_{3}$, cysteine and $\mathrm{NaBH}_{4}$ at 10 , 60 and 120 min after reaction start.

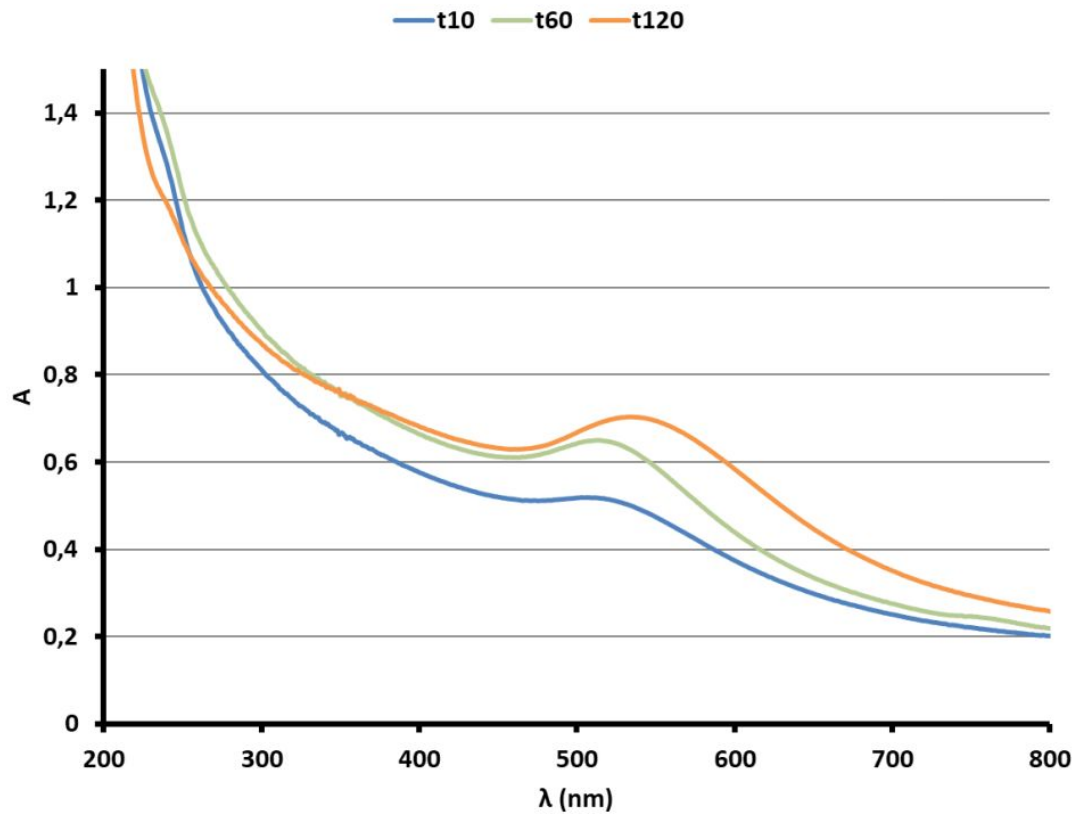

Figure S2. The UV/VIS spectra of the reaction mixture containing $\mathrm{HAuCl}_{4}$, cysteine and $\mathrm{NaBH}_{4}$ at 10, 60 and 120 min after reaction start. 


\section{NMR spectra}

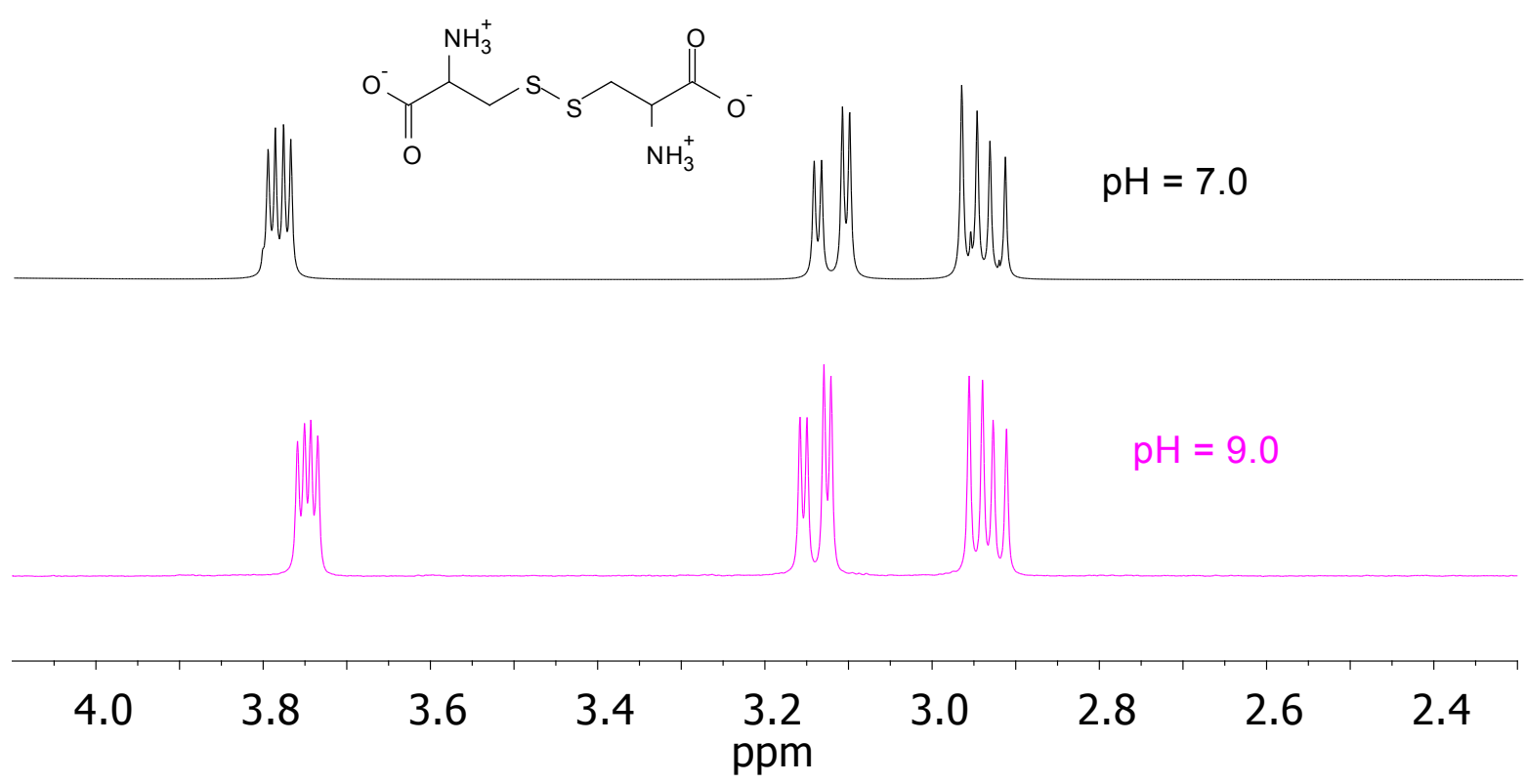

Figure S3. ${ }^{1} \mathrm{H}$ NMR spectra of pure cystine in water at different $\mathrm{pH}$ values.
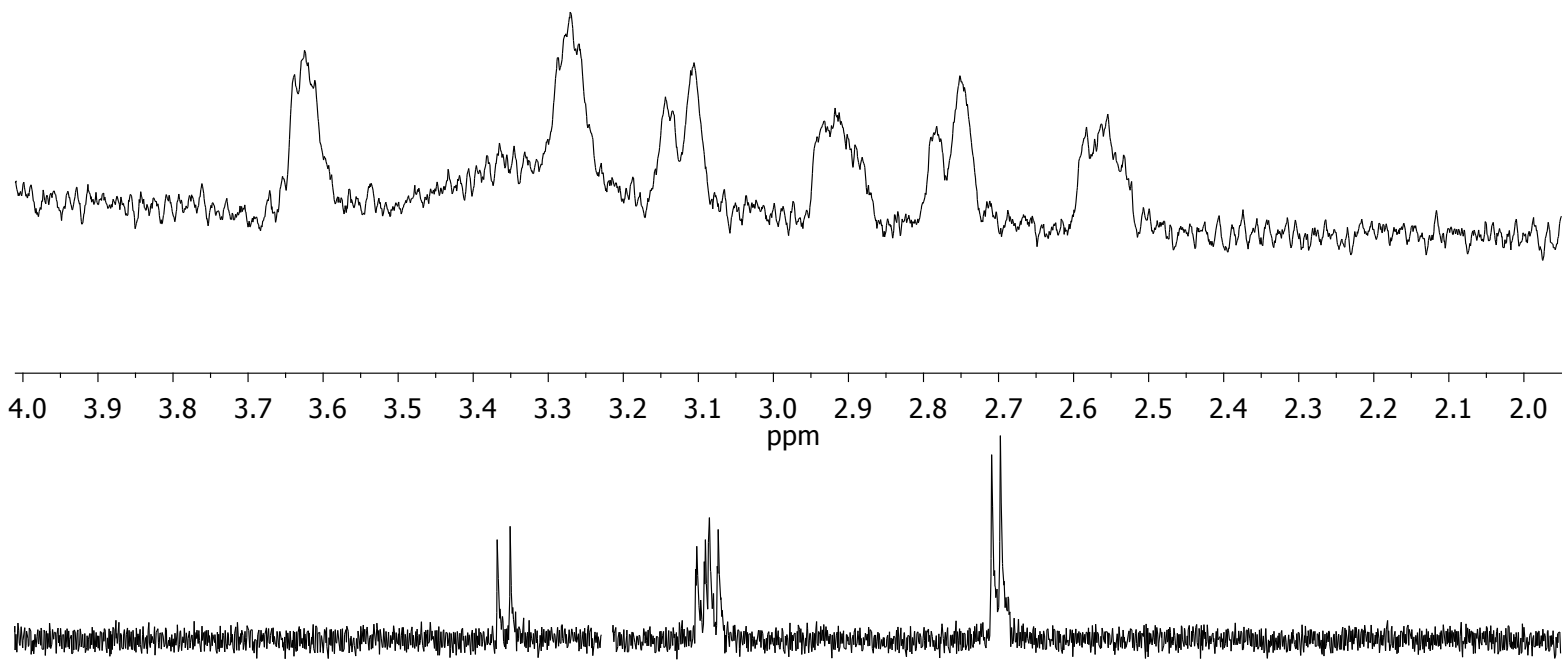

\begin{tabular}{lllllllllllllll}
\hline 198 & 192 & 186 & 180 & 58 & 54 & 50 & 46 & 42 & 38 & 34 & 30 & 26 & 22
\end{tabular}

Figure S4. ${ }^{1} \mathrm{H}$ (top) and ${ }^{13} \mathrm{C}$ NMR spectra (bottom) of cystine-functionalized silver nanoparticles in $\mathrm{D}_{2} \mathrm{O}$ after purification by centrifugation. 

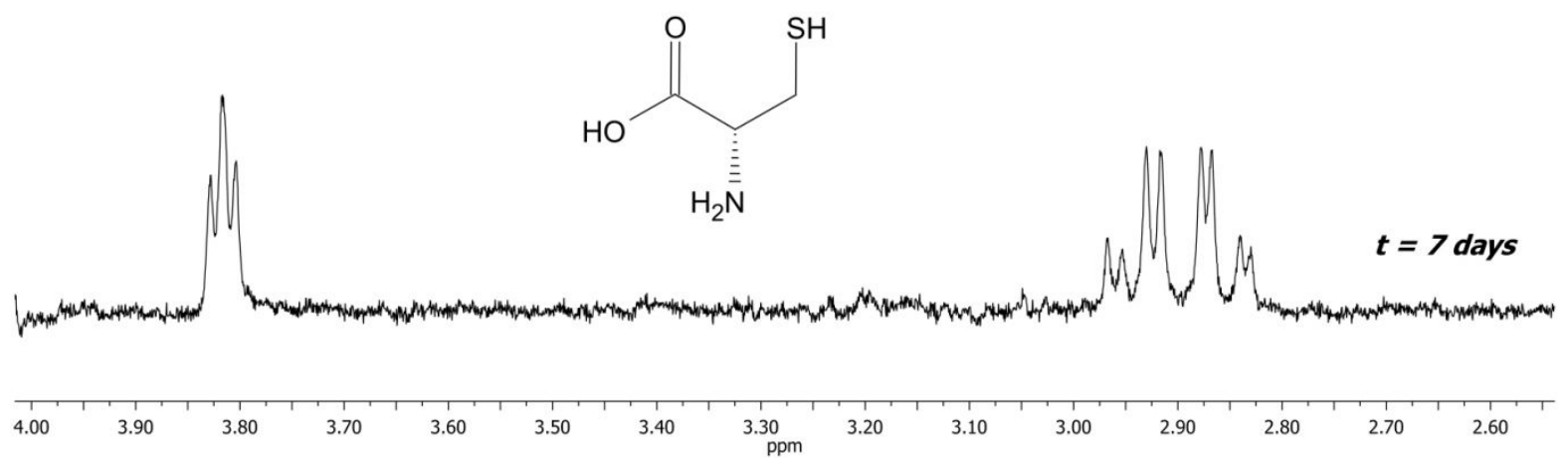

Figure S5. ${ }^{1} \mathrm{H}$ NMR spectra of the aqueous solution of cysteine $\left(5.6 \mathrm{mM}\right.$ in ultrapure water $/ \mathrm{D}_{2} \mathrm{O}$ added) taken after 7 days. No signals corresponding to (spontaneous) oxidation/dimerization process.

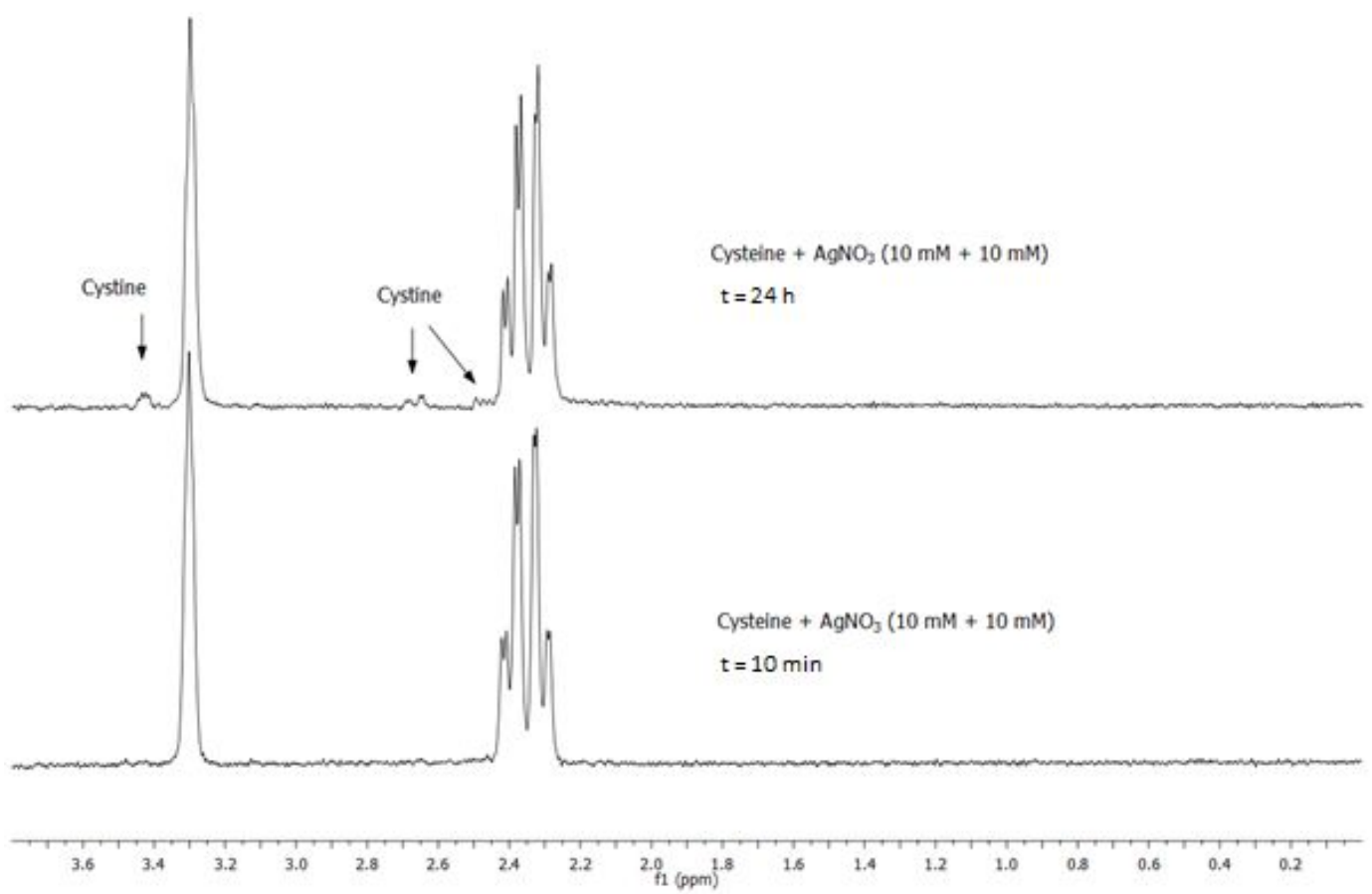

Figure S6. ${ }^{1} \mathrm{H}$ NMR spectra of the aqueous mixture of cysteine and silver nitrate (in equimolar concentrations), immediately after mixing and $24 \mathrm{~h}$ later. The arrows point to the signals of oxidized cysteine form, cystine. 


\section{Additional computational information}

\subsection{Images and energy tables}

A

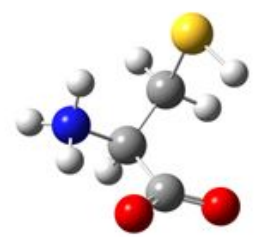

C

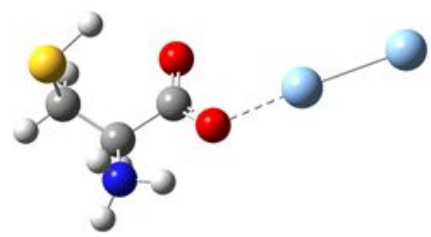

E

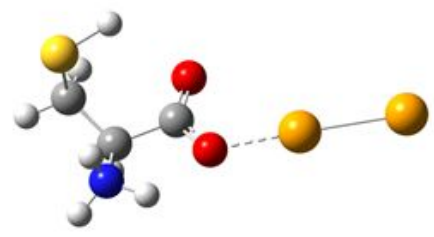

G

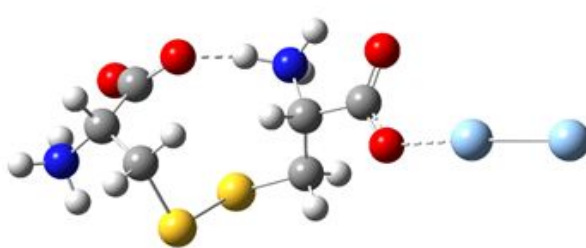

I

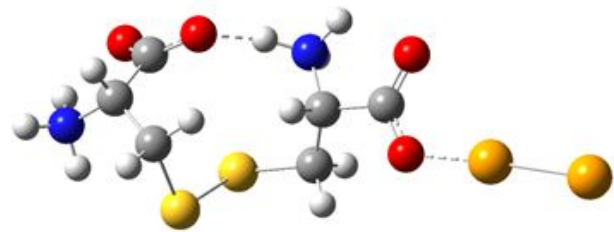

B

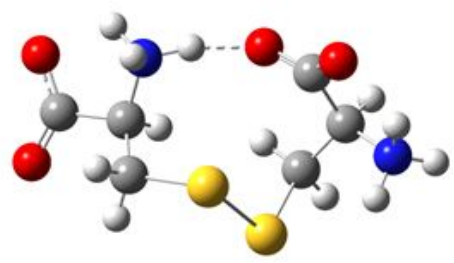

D

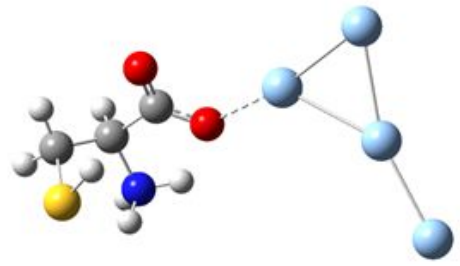

$\mathbf{F}$

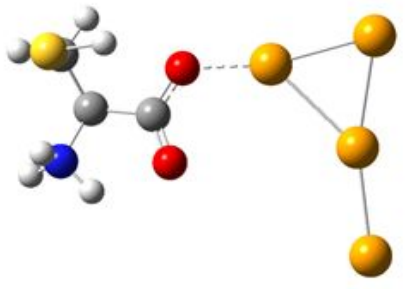

H

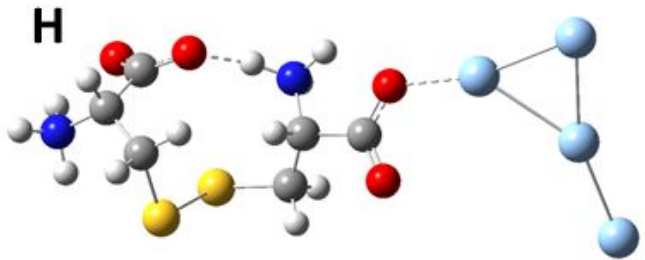

J

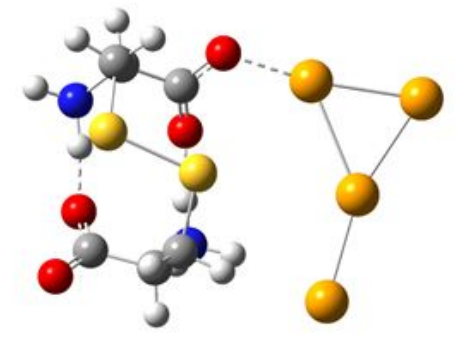

Figure S7. Fully optimized (at the B3LYP/LANL2DZ level) lowest energy structures of a) cysteine, b) cystine, c) cysteine- $\mathrm{Ag}_{2}$ complex, d) cysteine- $\mathrm{Ag}_{4}$ complex, e) cysteine- $\mathrm{Au}_{2}$ complex, f) cysteine- $\mathrm{Au}_{4}$ complex, g) cystine- $\mathrm{Ag}_{2}$ complex, h) cystiine- $\mathrm{Ag}_{4}$ complex, i) cystine- $\mathrm{Au}_{2}$ complex, and j) cystine- $\mathrm{Au}_{4}$ complex. 
Table S2. B3LYP/LANLD2Z calculated energies (in Hartree and eV) and experimental oxidation potential (in $\mathrm{mV}$ ) for selected thiols and their complexes with metallic dimers.

\begin{tabular}{|l|l|l|l|}
\hline Thiol & $\mathrm{E}_{\text {номо }}{ }^{\mathrm{a}}$ (Hartree) & \multicolumn{1}{|c|}{$\mathrm{E}_{\text {Hомо }}(\mathrm{eV})$} & \multicolumn{1}{|c|}{$\mathrm{E}^{\prime}{ }_{\text {exp }}(\mathrm{mV})$} \\
\hline thiophenol & -0.22766 & -6.14 & -300 \\
\hline homocysteine & -0.24376 & -6.58 & -256 \\
\hline cysteine & -0.24956 & -6.73 & -245 \\
\hline penicillamine & -0.25024 & -6.75 & -243 \\
\hline glutathione & -0.25245 & -6.81 & -240 \\
\hline cysteine- $\mathrm{Ag}_{4}$ complex & -0.25864 & -6.98 & -222 \\
\hline cysteine- $\mathrm{Ag}_{2}$ complex & $-0.25894^{\mathrm{b}}$ & -6.99 & $-222^{\mathrm{d}}$ \\
\hline cysteine- $\mathrm{Au}_{2}$ complex & $-0.25994^{\mathrm{c}}$ & -7.01 & $-219^{\mathrm{d}}$ \\
\hline cysteine- $\mathrm{Au}_{4}$ complex & -0.26086 & -7.01 & -219 \\
\hline
\end{tabular}

${ }^{a}$ The $3 p$-type orbital localized on the $\mathrm{S}$ atom. ${ }^{\mathrm{b}} \mathrm{HOMO}-2$ state. ${ }^{\mathrm{c}} \mathrm{HOMO}-6$ state. ${ }^{\mathrm{d}}$ Estimated value.

Table S3. Enthalpies, Gibbs free energies and relative energies calculated for singlet and triplet ground states at different levels. ${ }^{\text {a }}$

\begin{tabular}{|c|c|c|c|c|}
\hline \multirow[t]{2}{*}{ Ground states } & \multicolumn{2}{|c|}{ SMD-(U)B3LYP/LANL2DZ } & (U)PBE//(U)B3LYP & (U)PBE//(U)B3LYP + $\mathrm{H}_{\text {corr }}$ \\
\hline & $\mathrm{H}_{298}$ & $\mathrm{H}_{\text {corr }} \mathrm{b}$ & $\mathrm{E}+\Delta \mathrm{G}_{\text {solv }}$ & $\Delta \mathrm{H}_{298}$ \\
\hline Cystine (singlet) & -666.240499 & 0.221584 & -665.774025 & 0.0 \\
\hline Cystine (triplet) & -666.197360 & 0.220479 & -665.733384 & +103.8 \\
\hline Cystine- $\mathrm{Ag}_{2}$ (singlet) & -957.843423 & 0.226377 & -957.310428 & 0.0 \\
\hline Cystine- $\mathrm{Ag}_{2}$ (triplet) & -957.833730 & 0.225094 & -957.297877 & +29.6 \\
\hline Cystine- $\mathrm{Ag}_{4}$ (singlet) & -1249.438030 & 0.231940 & -1248.839645 & 0.0 \\
\hline Cystine- $\mathrm{Ag}_{4}$ (triplet) & -1249.464708 & 0.230966 & -1248.873956 & -92.6 \\
\hline Cystine- $\mathrm{Au}_{2}$ (singlet) & -937.228467 & 0.226401 & -936.753693 & 0.0 \\
\hline Cystine- $\mathrm{Au}_{2}$ (triplet) & -937.195597 & 0.225171 & -936.717555 & +91.7 \\
\hline Cystine- $\mathrm{Au}_{4}$ (singlet) & -1208.213542 & 0.232231 & -1207.732427 & 0.0 \\
\hline Cystine- $\mathrm{Au}_{4}$ (triplet) & -1208.217106 & 0.229910 & -1207.739672 & -25.1 \\
\hline
\end{tabular}

${ }_{a}^{a}(U) P B E / / U B 3 L Y P$ equals to (U)PBE-D3/LANL2DZ//SMD-(U)B3LYP/LANL2DZ $+\Delta G_{\text {solv }}\left((U) P B E-D 3, S C R F=\left(S M D\right.\right.$, solvent $=$ water)). ${ }^{b} H_{\text {corr }}$ : Thermal correction to Enthalpy. 
Table S4. Calculated energies for reactants, transition state structures, and products involved in the S-S dimerization of cysteine (see Scheme 2 in the main text) in water. ${ }^{a}$

\begin{tabular}{|c|c|c|c|c|c|c|c|c|}
\hline \multirow{2}{*}{\multicolumn{2}{|c|}{$\begin{array}{l}\text { Stationary points } \\
\mathbf{A g}_{\mathbf{n}}\end{array}$}} & \multicolumn{3}{|c|}{ B3LYP/LANLD2Z } & \multicolumn{2}{|c|}{ B1B95/LANLD2Z } & \multicolumn{2}{|c|}{ TPSS/LANLD2Z + GD3 ${ }^{b}$} \\
\hline & & \multirow{2}{*}{$\begin{array}{l}\begin{array}{l}H_{298} \\
\text { (Hartree) }\end{array} \\
-410.129815\end{array}$} & \multirow{2}{*}{$\begin{array}{l}H_{\text {corr }}{ }^{\mathrm{c}} \\
\text { (Hartree) } \\
0.144975\end{array}$} & $\begin{array}{l}\Delta H_{298} \\
(\mathrm{~kJ} / \mathrm{mol})\end{array}$ & $\begin{array}{l}E^{\mathrm{d}} \\
\text { (Hartree) }\end{array}$ & $\begin{array}{l}\Delta H_{298} \\
(\mathrm{~kJ} / \mathrm{mol})\end{array}$ & $\begin{array}{l}E^{\mathrm{d}} \\
\text { (Hartree) }\end{array}$ & $\begin{array}{l}\Delta H_{298} \\
(\mathrm{~kJ} / \mathrm{mol})\end{array}$ \\
\hline \multirow[t]{4}{*}{$n=0$} & cysteine- $\mathrm{H}_{2} \mathrm{O}$ & & & \multirow[b]{2}{*}{0} & \multirow{2}{*}{$\begin{array}{l}-409.998448 \\
-408.775159\end{array}$} & \multirow[b]{2}{*}{0} & \multirow{2}{*}{$\begin{array}{l}-410.267728 \\
-409.042758\end{array}$} & \multirow[b]{2}{*}{0} \\
\hline & $\begin{array}{l}\text { cysteine } \\
\text { sulfenic acid }\end{array}$ & -408.909926 & 0.125080 & & & & & \\
\hline & TS & -819.011999 & 0.269421 & +72.8 & -818.713362 & +156.5 & -819.271749 & +100.6 \\
\hline & cystine $-2 \mathrm{H}_{2} \mathrm{O}$ & -819.056909 & 0.273851 & -44.5 & -818.824412 & & -819.367486 & -139.7 \\
\hline \multirow[t]{6}{*}{$n=2$} & cysteine- $\mathrm{Ag}_{2}$ & -625.302947 & 0.124330 & \multirow{3}{*}{0} & -625.070723 & \multirow{3}{*}{0} & -625.214620 & \multirow{3}{*}{0} \\
\hline & $\begin{array}{l}\text { cysteine } \\
\text { sulfenic acid }\end{array}$ & -408.909926 & 0.125080 & & -408.775159 & & -409.042758 & \\
\hline & $\mathrm{H}_{2} \mathrm{O}$ & -76.408198 & 0.024515 & & -76.382323 & & -76.417668 & \\
\hline & $\mathrm{TS}_{\mathrm{Ag} 2}$ & -1110.590467 & 0.273559 & +70.4 & -1110.17059 & +140.3 & -1110.647091 & +72.4 \\
\hline & cystine $-2 \mathrm{H}_{2} \mathrm{O}$ & -819.056909 & 0.273851 & \multirow{2}{*}{-36.3} & -818.824412 & \multirow{2}{*}{-88.5} & -819.367486 & \multirow{2}{*}{-102.7} \\
\hline & $\mathrm{Ag}_{2}$ & -291.577987 & 0.004306 & & -291.441739 & & -291.350897 & \\
\hline \multirow[t]{6}{*}{$n=4$} & cysteine- $\mathrm{Ag}_{4}$ & -916.892764 & 0.130155 & \multirow{3}{*}{0} & -916.551064 & \multirow{3}{*}{0} & -916.609821 & \multirow{3}{*}{0} \\
\hline & $\begin{array}{l}\text { cysteine } \\
\text { sulfenic acid }\end{array}$ & -408.909926 & 0.125080 & & -408.775159 & & -409.042758 & \\
\hline & $\mathrm{H}_{2} \mathrm{O}$ & -76.408198 & 0.024515 & & -76.382323 & & -76.417668 & \\
\hline & $\mathrm{TS}_{\mathrm{Ag} 4}$ & -1402.204879 & 0.274534 & +15.8 & -1401.652683 & +123.0 & -1402.044593 & +53.7 \\
\hline & cystine $-2 \mathrm{H}_{2} \mathrm{O}$ & -819.056909 & 0.273851 & \multirow{2}{*}{-60.3} & -818.824412 & \multirow{2}{*}{-92.4} & -819.367486 & \multirow{2}{*}{-96.7} \\
\hline & $\mathrm{Ag}_{4}$ & -583.176971 & 0.009568 & & -582.919311 & & -582.743239 & \\
\hline \multicolumn{9}{|l|}{$A u_{n}$} \\
\hline \multirow[t]{6}{*}{$n=2$} & cysteine- $\mathrm{Au}_{2}$ & -604.681238 & 0.124619 & \multirow{3}{*}{0} & -604.432886 & \multirow{3}{*}{0} & -604.648167 & \multirow{3}{*}{0} \\
\hline & $\begin{array}{l}\text { cysteine } \\
\text { sulfenic acid }\end{array}$ & -408.909926 & 0.125080 & & -408.775159 & & -409.042758 & \\
\hline & $\mathrm{H}_{2} \mathrm{O}$ & -76.408198 & 0.024515 & & -76.382323 & & -76.417668 & \\
\hline & $\mathrm{TS}_{\mathrm{Au2} 2}$ & -1089.987099 & 0.276472 & +32.2 & -1089.550303 & +101.1 & -1090.101964 & +18.2 \\
\hline & cystine $-2 \mathrm{H}_{2} \mathrm{O}$ & -819.056909 & 0.273851 & -363 & -818.824412 & -499 & -819.367486 & -541 \\
\hline & $\mathrm{Au}_{2}$ & -270.956291 & 0.004302 & -50.3 & -270.78890 & & -270.765642 & -54.1 \\
\hline
\end{tabular}

a Solvation energies $\left(\Delta G_{\text {solv }}\right)$ calculated with SMD model (solvent=water) at the respective level of theory. ${ }^{b}$ The D3 version of Grimme's dispersion with the original D3 damping function. ${ }^{\mathrm{c}}$ Thermal correction to enthalpy. ${ }^{\mathrm{d}}$ Single point energy calculated for SMD-B3LYP/LANL2DZ geometries. 


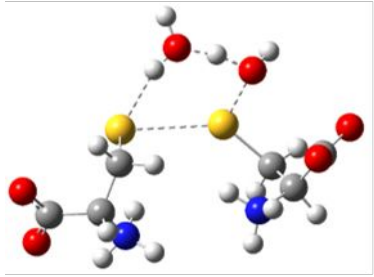

TS

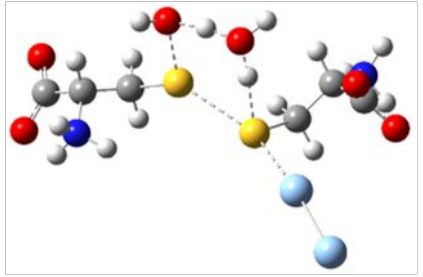

$\mathrm{TS}_{\mathrm{Ag} 2}$

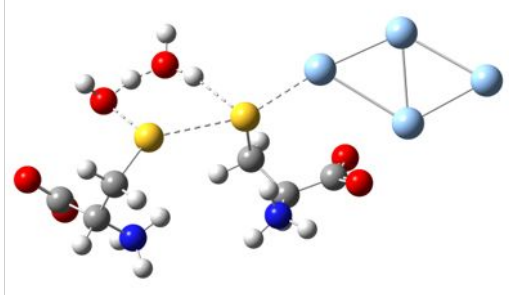

$\mathrm{TS}_{\mathrm{Ag} 4}$

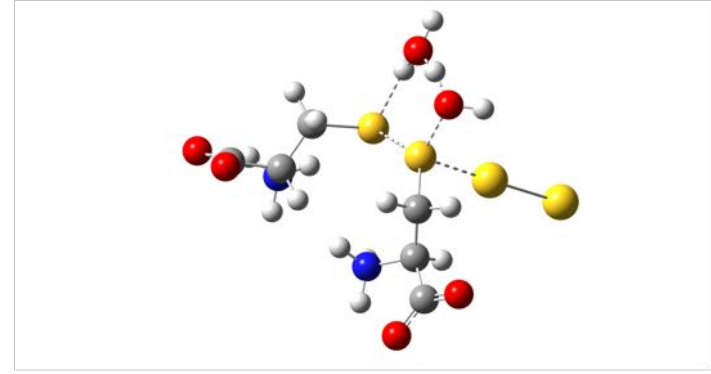

$\mathrm{TS}_{\mathrm{Au2}}$

Figure S8. Transition state structures TS (left) for the reaction between cysteine and cysteine sulfenic acid in water, and the transition state structures $\mathbf{T S}_{\mathrm{Ag} 2}, \mathbf{T S}_{\mathrm{Ag} 4}$, and $\mathbf{T S}_{\mathrm{Au} 2}$ for the analogous processes catalysed by $\mathrm{Ag}_{2}, \mathrm{Ag}_{4}$, and $\mathrm{Au}_{2}$ clusters, respectively.
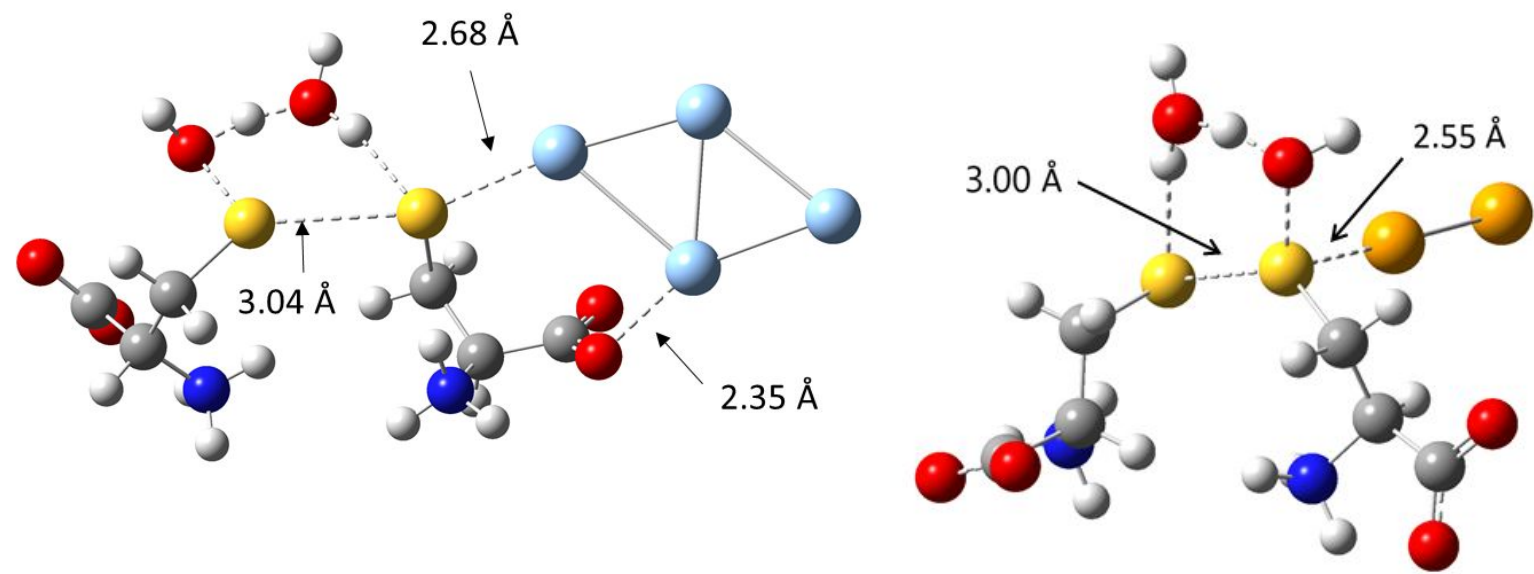

Figure S9. Fully optimized (at the B3LYP/LANL2DZ level) structures of transition states $\mathbf{T S}_{\text {Ag4 }}$ (left) and $\mathrm{TS}_{\text {Au2 }}$ (right) for the reaction between cysteine and cysteine sulfenic acid catalysed by clusters $\mathrm{Ag}_{4}$ and $\mathrm{Au}_{2}$, respectively. 


\section{4. $X Y Z$ coordinates of all structures from theoretical calculations}

\section{CYSTEINE}

Structure 1

N $0.385779001 .78311700-0.04669400$

H $1.241994001 .67124300-0.65332800$

C 0.398790000 .497082000 .76488400

H 0.872032000 .721382001 .72767700

C $-1.00461900-0.057387001 .03021500$

C $1.35379800-0.48078800-0.01324700$ H $-0.90886100-1.025157001 .52215000$ H -1.593565000 .614747001 .65891300$ S - $2.05002200-0.27957200-0.54373000$ O $2.099585000 .10354000-0.90192900$ H - $1.32262100-1.31604400-1.08423000$ H 0.425819002 .632583000 .52405400 H -0.44662900 $1.81191100-0.65521700$ O $1.31840500-1.712637000 .31385500$ $\mathrm{H}=-333,696333$ Hartree $\mathrm{G}=-333,738497$ Hartree

\section{Structure 2}

N 0.140544001 .595403000 .26251300 H $0.970822001 .80946600-0.35257600$ C 0.264893000 .093849000 .47117700 H $0.12429400-0.131023001 .53051900$ C $-0.75027500-0.68265800-0.38396500$ C $1.72876300-0.277818000 .03102200$ H - $0.57954200-0.52650200-1.45301000$ H - $0.65834800-1.74617100-0.16402100$ S - $2.56064700-0.15566800-0.12151600$ O $2.12464800-1.459330000 .29892700$ H -2.68093000 -0.56513800 1.18751100 H 0.239515002 .112585001 .14238500 H - $0.756879001 .85728000-0.16615300$ O $2.358767000 .67334700-0.58985200$ $\mathrm{H}=-333,69509$ Hartree $G=-333,737428$ Hartree

Structure 3 N 0.331744001 .776783000 .03614100 H $1.167530001 .70410700-0.60782000$ C 0.395761000 .454318000 .78178800 H 0.864668000 .642353001 .75405200
C - $0.98215800-0.169260001 .02612900$ C $1.38516800-0.44753100-0.04453400$ H - $0.83698200-1.164290001 .44576000$ H -1.58336000 0.432965001 .70966900 S - $1.97473900-0.37637700-0.59741100$ O $2.069606000 .20058200-0.93961300$ H -3.06160000 0.38892900 - 0.24454500 H 0.384877002 .597442000 .64630600 H -0.52313400 $1.82393400-0.53835000$ O $1.43901800-1.683837000 .25964100$ $\mathrm{H}=-333,694186$ Hartree $G=-333,737155$ Hartree

Structure 4

$\mathrm{N}-0.146133001 .60082600-0.22352200$ H -0.21690600 2.13185500 -1.09777900 C - $0.263550000 .10157800-0.45826100$ H - $0.12316700-0.10485200-1.52173100$ C $0.74976600-0.682814000 .38660800$ C - $1.72933300-0.28252700-0.03238600$ H $0.60407000-0.502497001 .45452800$ H $0.63045300-1.746060000 .18049400$ S $2.54268200-0.17394100-0.05267800$ O - $2.11304200-1.46721300-0.30185800$ H $3.06699300-0.272911001 .21475900$ H 0.735040001 .856687000 .24027300 H -0.99768600 1.802235000 .36833600 O - 2.374468000 .666638000 .57596500 $\mathrm{H}=-333,693168$ Hartree $G=-333,735972$ Hartree

Structure 5 N 0.314205001 .664291000 .04797000 H $1.360118001 .79616600-0.10391100$ C 0.253788000 .171356000 .35376600 H 0.067686000 .060701001 .42555600 C - $0.81687100-0.57676600-0.44188400$ C $1.70739700-0.366668000 .05717300$ H - $0.73460200-0.39661900-1.51710600$ H - $0.68230500-1.64422300-0.26123900$ S - $2.60772200-0.216231000 .10082900$ O $1.88791600-1.624718000 .12626200$ H -2.82003400 $0.91798900-0.64988200$ 
H -0.031591002 .247582000 .81567200$ H $-0.185728001 .91808000-0.81073700$ O $2.572671000 .56752400-0.20897900$ $\mathrm{H}=-333,693273$ Hartree $G=-333,735685$ Hartree

Structure 6

N - $1.570097001 .55687500-0.10408300$ H - $1.730592002 .18949100-0.89356900$ C - $0.425698000 .57300600-0.35833400$ H - $0.190982000 .61331500-1.42627600$ C 0.822711000 .944243000 .46224700 C - $1.03313900-0.84172400-0.02832900$ H 1.096776001 .989616000 .29812200 H 0.659202000 .775851001 .53019800 S $2.34511600-0.05029400-0.08295600$ O - $0.23453400-1.84267900-0.00696400$ H $1.64828900-1.25361100-0.06436100$ H -1.42846800 2.117073000 .74403900 H - 2.390688000 .899839000 .03889900 O - $2.31271000-0.842088000 .17887900$ $\mathrm{H}=-333,693457$ Hartree $G=-333,735031$ Hartree

Structure 7

N -1.51025200 $1.59543500-0.10250300$ H - 2.360658000 .965871000 .02349800 C - $0.415296000 .55504300-0.35096500$ H - $0.168965000 .58880300-1.41607300$ C 0.837800000 .862194000 .48413500 C - $1.09754100-0.82454100-0.03069900$ H 1.135715001 .904267000 .34828500 H 0.669612000 .666630001 .54569200 S $2.31391000-0.20053200-0.08805900$ O - $0.35462800-1.857773000 .00683200$ H $3.228166000 .82990400-0.13455100$ H - $1.630739002 .24194500-0.88764700$ H -1.356110002 .140107000 .75337000$ O $-2.38507100-0.748881000 .15305100$ $H=-333,689065$ Hartree $G=-333,731606$ Hartree

Structure 8

N -1.44057000 $1.61259500-0.25998200$ H -1.85789600 $1.89664500-1.15260900$
C $-0.386726000 .51550900-0.44486300$ H -0.08721300 $0.48970100-1.49402000$ C 0.827799000 .828955000 .46007700 C - $1.13796400-0.80776300-0.04524900$ H 1.121061001 .876937000 .35271500 H 0.599040000 .635731001 .51154200 S $2.36794600-0.206076000 .08566400$ O -0.62813500 - $1.91961000-0.39198400$ H $2.774616000 .49845400-1.02531400$ H -1.08930100 2.448934000 .21735300 H -2.16651600 1.098993000 .32528600 O - $2.23631400-0.599960000 .62879800$ $\mathrm{H}=-333,688315$ Hartree $G=-333,731314$ Hartree

CYSTINE

Structure 1

S $0.13685100-1.103934001 .45453000$

S $1.22368500-2.00772300-0.34331200$ O -4.06144900 $1.33240000-0.51509600$ O 2.625377001 .842870001 .06494300 O $-4.06351300-0.97157700-0.77782300$ O $0.776084001 .97650700-0.32124900$ N -1.68639600 1.490126000 .51923700 N $3.80218100-0.06346000-0.22517400$ C - $2.012011000 .12916600-0.06092400$ C $2.599030000 .51204100-0.95288600$ C - $1.68333600-1.025840000 .88415300$ C $1.65203600-0.55807100-1.51004500$ C -3.52989000 0.15168300 -0.48205400 C 1.929607001 .529747000 .03331900 H - $1.431026000 .03378100-0.98377500$ H $2.975575001 .09757100-1.79912200$ H -2.23666800 -0.95726900 1.82861500 H - $1.93314400-1.971460000 .40398000$ H $0.73724600-0.06866600-1.84057400$ H $2.10083600-1.08647300-2.35865800$ H -2.45069300 2.100245000 .15339900 H - 0.717067001 .810587000 .22450500 H $4.64264800-0.11535000-0.80965100$ H $3.58889300-1.003756000 .14285000$ H -1.74184100 1.494704001 .54344900 H 3.941551000 .591971000 .58146300 
$H=-666.221116$ Hartree

$G=-666,281793$ Hartree

Structure 2

S - 1.948784001 .900033000 .29460200

S - $0.140383001 .52698700-1.06808700$

O - $0.48948800-1.788569000 .31006200$

O $3.99709300-1.212724000 .52089300$

O -1.80814800 -1.83846100 -1.59328800

O 4.050228001 .070695000 .92034100

N -2.28554900 -0.88160000 1.90123700

N $1.83728000-1.23045600-0.87514700$

C - $2.74101500-0.924127000 .44881800$

C $2.294852000 .17055700-0.51517400$

C - $3.140385000 .46034400-0.09259800$

C 1.215948001 .000495000 .18700900

C - $1.59038800-1.58012100-0.37518600$

C 3.569736000 .005319000 .40807700

H -3.61763600 - 1.574863000 .38472300

H $2.630981000 .67129300-1.42960000$

$\mathrm{H}-3.274717000 .39245100-1.17275000$

H - 4.074872000 .807560000 .36204600

H 0.737299000 .440485000 .99096000

H 1.671574001 .908519000 .57945700

H - $1.34314600-1.344075001 .87648300$

H - 2.92276900 - 1.378022002 .53209400

H $1.73554000-1.36095900-1.88516100$

H $0.91723900-1.50968900-0.40182100$

H - 2.162242000 .085326002 .23354600

H $2.61732200-1.82829100-0.50657400$

$\mathrm{H}=-666.219869$ Hartree

$\mathrm{G}=-666,280118$ Hartree

Structure 3

S - $1.50738700-2.07717500-0.06578300$ S $0.39901500-1.00047800-0.73043500$

O -1.83907800 $2.11914700-1.02327100$ O $3.373353000 .93898500-1.23142400$ O -0.84551100 1.636289001 .01160200 O $4.18458300-0.98587800-0.22111300$ N -3.62076700 $0.25055700-0.90463600$ N 1.796434001 .622633000 .71189900

C - 2.880173000 .469952000 .40373500

C 2.404643000 .241401000 .87698300

C - $2.40399100-0.829386001 .06177000$
C $1.34862600-0.873529000 .93259000$ C - 1.741568001 .498910000 .09531100 C $3.426854000 .03965600-0.29845600$ H -3.573990000 0.960008001 .09584900 H 2.954716000 .207834001 .82210900 H - $1.75456300-0.571226001 .89835000$ H -3.24551800 -1.42314100 1.43523800 H $1.84129900-1.829077001 .11273100$ H $0.61238100-0.673413001 .71333700$ H -4.63959100 $0.23712100-0.79418200$ H -3.31732400 -0.62808700 -1.35230800 H 2.201862002 .282217001 .38371400 H 0.730852001 .656819000 .80859500 H -3.31599900 $1.05590400-1.50264800$ H $2.097045001 .90478200-0.25008100$ $\mathrm{H}=-666.218970$ Hartree $G=-666,279503$ Hartree

Structure 4 S - $0.520524002 .19836600-0.77569600$ S 0.982658001 .788910000 .88871000 O $-4.29017400-0.40444200-0.94319100$ O $2.52301200-2.22957200-1.17576900$ O -3.60640700 -1.83125000 0.75124500 o $0.64857100-1.53558100-0.03741300$ $\mathrm{N}-1.42814700-0.583967001 .32398100$ N $4.21712500-0.781878000 .03701200$ C - 2.303882000 .175497000 .34367000 C $2.77497100-0.421144000 .40687300$ C - $1.554925000 .60264700-0.91892300$ C $2.485628001 .04010100-0.01228900$ C -3.51697700 -0.77724700 0.00264500 C $1.89631900-1.48831400-0.32910100$ H -2.71840900 1.050664000 .85445300 H $2.66322000-0.530454001 .48771700$ H -0.91360400 -0.19249700 -1.30161600 H -2.29546900 $0.87780000-1.67744300$ H 3.319986001 .684662000 .28111600 H 2.31859900 $1.13924800-1.08738400$ H - $1.17117700-0.023341002 .14001500$ H - $0.54189900-0.942217000 .86076800$ H $4.72846200-1.186970000 .82876800$ H $4.755692000 .01275000-0.32431300$ H - $2.03484600-1.393375001 .59973300$ H $4.08562200-1.51425200-0.71322400$ 
$H=-666.217268$ Hartree

$G=-666,279515$ Hartree

Structure 5

S - $0.829857002 .29526500-0.52648200$ S 0.960855002 .297735000 .89211500

O -3.33007200 -2.21839500 0.37988300 O $3.26489200-2.231373000 .03816600$ O -1.17870300 -1.79565000 1.10656300 O $3.33553900-0.86934700-1.83837900$ N -3.48246400 - $0.27548900-1.29137300$ N $1.36915000-0.975905001 .24766000$ C - $2.17780300-0.27711200-0.50184000$ C $1.75023800-0.34143400-0.07286500$ C - 1.970322001 .008442000 .32077900 C 2.202885001 .115744000 .05124500 C - $2.23562300-1.550456000 .41772900$ C $2.89719100-1.22707000-0.69377000$ H - $1.36066000-0.41644600-1.21202600$ H $0.88820800-0.39907100-0.74345700$ H -2.91170000 1.522095000 .53669100 H -1.49393400 0.768303001 .27197000 H 3.091879001 .217352000 .68601400 H $2.430545001 .50574000-0.94053300$ H - $4.016245000 .59466700-1.19338000$ H -4.01532200 -1.08182700 -0.86932700 H $0.36189400-1.317116001 .22681500$ H $1.48586200-0.338574002 .04246000$ H -3.33313900 - $0.45202100-2.29089100$ H $2.03717700-1.781901001 .32399000$ $\mathrm{H}=-666.217664$ Hartree $G=-666,278781$ Hartree

Structure 6 S $0.316149001 .72669800-1.11106400$ S -0.31681200 1.724646001 .11433900 O $1.15760800-1.564407001 .16409300$ O -1.15704200 -1.56132300 -1.16781600 O $2.95029400-0.179265001 .60195200$ O - $2.95047100-0.17596700-1.60190500$ N $1.42779500-1.39633200-1.51286500$ N -1.42716900 -1.40034400 1.50967300 C $2.28554900-0.45788600-0.69058100$ C - $2.28531900-0.460129000 .68980800$ C $2.083510001 .02271600-1.05381600$
C - 2.083740001 .019590001 .05666400 C $2.11519700-0.749037000 .83008700$ C - $2.11500300-0.74734500-0.83160000$ H $3.33040800-0.68644200-0.93435400$ H - $3.33008000-0.689627000 .93314200$ H $2.674789001 .62520200-0.36231200$ H $2.428634001 .22916800-2.07427900$ H -2.67596000 1.623494000 .36720700 H -2.42807700 1.223249002 .07794400 H $1.71028000-2.36353000-1.31580100$ H $1.55332200-1.22412700-2.51712300$ H - $1.55240100-1.230398002 .51436500$ H - $0.37178600-1.353861001 .28315400$ H $0.37242600-1.35052100-1.28651300$ H -1.70961500 -2.36714300 1.31053200 $\mathrm{H}=-666.220608$ Hartree $G=-666,278894$ Hartree

Structure 7

S $1.143739001 .45202700-0.71828100$ S - 0.787455002 .399860000 .06166000 o $3.07746900-2.11189700-0.72360700$ o -3.63826100 - $2.08636600-0.19514600$ O $1.11534700-1.867628000 .47734000$ O $-4.50908900-0.116363000 .66392400$ N 4.171856000 .088636000 .03727000 $\mathrm{N}-1.25187400-1.21901100-0.57601600$ C $2.89816100-0.253327000 .80806100$ C - $2.12211000-0.227072000 .17111100$ C 1.921587000 .914382000 .95700400 C - $2.147721001 .16802000-0.45565600$ C 2.30039000 - 1.526311000 .11558600 C - $3.57332500-0.858532000 .21390400$ H $3.20782600-0.549377001 .81645800$ H -1.76923500 -0.17629400 1.20570000 H 1.103878000 .587530001 .59845400 H 2.402247001 .789902001 .39842600 H - $2.180103001 .13153400-1.54811200$ H -3.04959800 $1.67780300-0.09796700$ H $4.32475200-0.76696100-0.55578000$ H 4.974780000 .250720000 .65425200 H -0.34756300 - $1.47181800-0.08535700$ H -1.01291700 -0.88693100 -1.51607700 H $4.062893000 .90725500-0.57290100$ H -1.88298500 -2.05584300 -0.66008900 
$H=-666.215501$ Hartree

$G=-666,277636$ Hartree

Structure 8

S - 0.817539002 .424235000 .02440600

S $1.113128001 .45084300-0.71550200$

O $-4.44289600-0.185811000 .77615300$

O $1.12774100-1.901589000 .38038500$

O -3.58853900 -2.10640800 -0.20299900

O $3.16086500-2.08165600-0.70678600$

N -1.23197000 -1.19691600 -0.65066200

N 4.139165000 .150384000 .11921100

C - $2.08549800-0.233048000 .15082100$

C $2.85001200-0.248486000 .83340100$

C - $2.170660001 .16854900-0.45686900$

C 1.833540000 .885187000 .97601500

C -3.52120000 -0.894092000 .24954400$

C $2.32211800-1.525220000 .09160600$

H -1.68714900 -0.18667600 1.16887600

H $3.13413700-0.559941001 .84496400$

H - 2.24235500 $1.14214700-1.54759200$

H -3.06911400 $1.65286900-0.05807600$ H 0.999275000 .515614001 .57187400

H 2.26953900 1.760205001 .46230900

H - $0.32555500-1.47466500-0.17721700$

H -0.99921400 - $0.82694000-1.57785400$

H 4.902887000 .360610000 .77023400

H $4.016730000 .95561400-0.50624900$

H - $1.86816300-2.02816300-0.75668400$

H $4.36194600-0.69982000-0.45804000$

$\mathrm{H}=-666.215488$ Hartree

$\mathrm{G}=-666,277152$ Hartree

Structure 9

S $1.05505200-1.91551600-0.45886800$

S - $1.05655800-1.915433000 .45940400$

O $1.309444001 .58065700-0.96251800$

O -1.30822800 1.581668000 .96184800

O $3.506718000 .89150400-1.04116500$

O -3.50583800 0.89370800 1.04098100

N 1.129675001 .089493001 .72332100

N -1.12864200 $1.08842600-1.72382700$

C 2.245835000 .410320000 .95063400

C - $2.245498000 .41089300-0.95069400$

C $2.11384300-1.124496000 .91638000$
C - $2.11513700-1.12403400-0.91568000$

C $2.374539001 .01972500-0.47369000$

C - 2.373625001 .021055000 .47335700

H 3.171357000 .627753001 .49428300

H -3.17080600 $0.62903600-1.49442500$

H $3.10125500-1.545820000 .70547000$

H 1.77500400 -1.53015300 1.87361700

H -3.10289900 - $1.54418200-0.70404200$

H -1.77726600 -1.53051300 -1.87290200

H 0.184188001 .174184001 .21365800

H 1.409524002 .054552001 .93726400

H -1.40782900 2.05338800 -1.93908000

H - $0.961456000 .61249600-2.61818700$

H 0.962548000 .614724002 .61830800

H -0.18325500 1.17321700 -1.21401300

$\mathrm{H}=-666.217001$ Hartree

$G=-666,277423$ Hartree

Structure 10

S $0.93934000-1.41392500-0.45664900$

S - $1.07117900-2.210702000 .26265600$

O $3.490386000 .81903900-1.43747800$

O -0.95333600 1.269220000 .54079400

O $4.54675600-0.296839000 .30096700$

O - $2.860165002 .37352800-0.14990800$

N 1.576436001 .813282000 .02221900

$\mathrm{N}-4.384648000 .33981100-0.25795900$

C 2.403856000 .754080000 .72525900

C - $2.97770300-0.013987000 .23608300$

C $1.55921800-0.473031001 .10053300$

C - $2.35794200-1.12973600-0.63289000$

C $3.603507000 .39286800-0.21799700$

C - 2.187578001 .335244000 .20502700

H 2.800819001 .173485001 .65461700

H -3.06955400 -0.35004400 1.27190300

H $2.17154900-1.166720001 .67742700$

H $0.66760900-0.186783001 .65918000$

H -3.12510800 -1.85948400 -0.91320400

H -1.89603900 -0.73868700 -1.54191400

H $1.855185001 .72798400-0.97898500$

H 1.837239002 .751225000 .34291900

H -4.33381400 $1.38886000-0.36859100$

H -5.11284300 0.079817000 .41501900

H 0.532640001 .678160000 .16803700

H -4.61005500 -0.08765600 -1.16343200 
$\mathrm{H}=-666.214618$ Hartree

$\mathrm{G}=-666,27681$ Hartree

Structure 11

S $0.96100500-1.576896001 .24319500$

S - $0.48115100-2.25081300-0.42646400$

O 3.304368002 .197215000 .17713300

O -3.66840800 1.951579000 .29109200

O 1.088874001 .823939000 .67546700

O -4.21345200 0.27235500 - 1.21158700

N $3.536693000 .33447400-1.55283700$

N - 1.388326000 .969195001 .00334500

C $2.289865000 .18238000-0.67760100$

C - $2.000930000 .25537600-0.18339000$

C $2.46315300-1.072076000 .20521200$

C - $2.06130700-1.26264200-0.02762900$

C 2.210461001 .519459000 .13152300

C - $3.442827000 .87193800-0.38775600$ H $1.421731000 .06746800-1.32936900$

$\mathrm{H}-1.416688000 .52283700-1.06990700$

H $2.71468200-1.93648600-0.41555700$ H $3.25282200-0.933466000 .95247000$ H -2.40994000 -1.56314400 0.96445200 H -2.76591100 -1.64886200 -0.77196000 H $4.064427001 .11192000-1.07765900$ H $3.295893000 .62817900-2.50650600$ H -2.02519200 1.78997500 1.13788200 $\mathrm{H}-0.379316001 .275711000 .83526800$ H $4.10520400-0.51778200-1.61070600$ H -1.41550100 0.39399900 1.85132400 $\mathrm{H}=-666.216364$ Hartree

$\mathrm{G}=-666,277106$ Hartree

Structure 12

S $0.88159200-1.37460600-0.51293600$ $S-1.10451000-2.215080000 .23317100$ O $4.52099200-0.380185000 .19977400$ O -2.67109200 $2.33635600-0.37730900$ O $3.444564000 .88800700-1.41766600$ O -0.92984200 1.254316000 .68739900 N 1.595009001 .809996000 .16584800 N -4.36358100 $0.44131500-0.20181800$ C 2.418418000 .686730000 .76614800

C - 2.969311000 .025600000 .28032200 C $1.55884900-0.546112001 .08419600$
C - $2.42486900-1.12562600-0.59616900$ C $3.581142000 .36838300-0.23740800$ C - 2.110838001 .328019000 .19294900 H 2.853691001 .031220001 .70898300 H -3.05052500 -0.29806400 1.31982000 H $2.17091400-1.286855001 .59946900$ H $0.68692500-0.279639001 .68225200$ H -3.22176500 - $1.84912800-0.80132500$ H - $2.02404600-0.76863800-1.54758000$ H $1.856848001 .80212100-0.84381800$ H 1.872644002 .715050000 .55881200 H -5.04929200 0.442699000 .56120300 H - $4.72568000-0.14658900-0.96050700$ H 0.548159001 .675922000 .31637400 H -4.20850100 $1.42778700-0.54347900$ $\mathrm{H}=-666.214633$ Hartree $G=-666,27622$ Hartree

Structure 13

S $1.63220400-1.703795000 .87532200$

S - $0.07155100-1.97575500-0.65987300$ o $1.761667001 .11017500-2.17886300$ O -3.93197500 1.488372000 .56359300 O $0.878995002 .10482200-0.28188400$ O $-4.35036600-0.54230900-0.47371100$ N 2.695062001 .313920001 .35090600 $\mathrm{N}-1.384411001 .181610000 .77930300$ C $2.888806000 .77673800-0.06083100$ C - $2.032897000 .14194000-0.11034600$ C $2.96957900-0.75802800-0.10414600$ C - $1.63672600-1.300636000 .20383100$ C $1.742167001 .37082400-0.94208100$ C -3.59494900 0.36613700 0.01049700 H $3.836634001 .17063300-0.44079700$ H -1.76348900 $0.38861100-1.14307200$ H $2.94075600-1.08808200-1.14347200$ H 3.89784700 -1.11514200 0.35335600 H - $1.55172800-1.499573001 .27583700$ H - 2.41897700 -1.95056200 -0.20066500 H 1.925673002 .018089001 .24300500 H 3.542665001 .744591001 .73403400 H -2.15821700 1.870546000 .93644600 H -0.531256001 .621845000 .32958500$ H 2.365524000 .580156001 .99394500 H -1.11287300 0.792640001 .68852400 
$\mathrm{H}=-666.214131$ Hartree

$\mathrm{G}=-666,275699$ Hartree

Structure 14

S $0.27835300-1.952435000 .69296000$

S -1.41968400 -1.92460700 -0.84477100

O $4.48288000-0.35396200-0.58584100$

O -2.74567600 $2.03101300-1.21221200$

O $3.808506001 .81721400-0.12958300$

O -0.57552200 $1.48117300-0.65363100$ N 1.546258001 .213137000 .93969600

N -3.71433800 1.107366001.03813900

C $2.38081600-0.004384000 .59670100$

C - 2.417081000 .444329000 .56485000

C $1.63092300-1.00105400-0.28863700$

C - $2.78696200-0.959999000 .04421800$

C $3.685754000 .52686100-0.11830600$

C - $1.855100001 .40332300-0.53245600$ H $2.70167100-0.482901001 .52766700$ $\mathrm{H}-1.732259000 .369569001 .41080800$ H $1.12797900-0.50800000-1.12326200$ H $2.33359900-1.74559400-0.66092200$ $\mathrm{H}-3.14304300-1.581387000 .86974400$ H -3.56656300 -0.90198700 -0.72512200 H 0.663958001 .285247000 .34181100 H 2.190407002 .008410000 .70745700 H -4.41810700 0.436003001 .36656400 $\mathrm{H}-4.057712001 .626195000 .19976500$ H 1.285610001 .243768001 .92873400 H -3.53928000 1.78187600 1.79279600 $\mathrm{H}=-666.214007$ Hartree $\mathrm{G}=-666,275222$ Hartree

Structure 15 S $0.90611800-1.699381000 .54583000$ S -0.29041900 -1.10461200 -1.30461900 O $4.26838100-0.170973000 .66648300$ O -3.60284300 $1.72258900-0.21915900$ O $3.301148000 .40518900-1.36245900$ O -1.30907100 1.76673400-0.01294200 N $1.333101001 .76053000-0.34430400$ $\mathrm{N}-3.94925400-0.593310000 .80039200$ C 2.139799000 .981862000 .68041400 C - $2.49142300-0.320822000 .42432600$ C $1.28799900-0.050719001 .43541500$
C - $2.08078600-1.28364700-0.70901100$ C $3.359503000 .34550600-0.06913500$ C - 2.454792001 .193137000 .02679200 H 2.518662001 .687874001 .42607100 H - $1.87229800-0.499150001 .30534600$ H $1.85370200-0.403829002 .30348200$ H 0.350413000 .388900001 .78030600 H -2.20914500 -2.32044800 -0.38766200 $\mathrm{H}-2.66918200-1.11150000-1.61791200$ H $0.283512001 .67342600-0.22386200$ H $1.659693001 .38000400-1.25968800$ H -4.08745700 -0.58287500 1.81756500 H -4.30896900 -1.48160200 0.43390100 H $1.577876002 .75584300-0.31484300$ H -4.45763800 0.22649200 0.37711800 $\mathrm{H}=-666.214553$ Hartree $\mathrm{G}=-666,275269$ Hartree

Structure 16 S - $1.98633200-1.83313600-0.36879100$ S $0.25305100-2.026510000 .11816100$ O - $0.753009002 .15800900-0.41779100$ O $3.41449500-1.318967000 .48647500$ O -0.66177200 1.419565001.77305400 O 4.088664000 .892983000 .28173400 N -3.02949500 $1.13132200-0.99701500$ N $1.838192001 .67651900-0.89196900$ C - 2.631121000 .906448000 .45421800 C $1.787901000 .33543100-0.17817100$ C - $2.70063800-0.574505000 .87738400$ C $1.04777900-0.71451300-1.01758400$ C - 1.221837001 .546447000 .64220400 C $3.23419300-0.075749000 .21988000$ $\mathrm{H}-3.332524001 .465747001 .08216500$ H 1.236317000 .529804000 .74835300 H -2.18937500 -0.69380400 1.83343300 $\mathrm{H}-3.74003500-0.901634000 .98219500$ H $0.23448800-0.27464700-1.59845800$ H 1.72262500 -1.25460300 -1.68405000 H - 2.32381900 $1.82319800-1.34669700$ H -3.98659400 $1.48216600-1.10314800$ H $2.033790001 .57432000-1.89410800$ H $2.633655002 .18532900-0.46701800$ H -2.91949400 0.26455800 -1.54519700 H $0.897903002 .14494900-0.74985500$ 
$H=-666.215603$ Hartree

$G=-666,275355$ Hartree

Structure 17

S $0.48832300-1.079414001 .02941900$

S - $0.48830800-1.07921600-1.02954900$

O $3.577601002 .17279800-0.71708700$

O -3.57833100 2.17239800 0.71823800

O 5.197452000 .699136000 .05621500

O -5.19721000 $0.69919300-0.05790800$

N $3.71601300-1.343612000 .24695900$

N -3.71555400 -1.34390300 - 0.24651500

C $2.93162600-0.11200400-0.18689300$

C - $2.93156600-0.111985000 .18717900$

C 1.825674000 .262231000 .80251300

C $-1.825774000 .26235400-0.80243500$

C $3.996778001 .04490700-0.30170600$

C -3.99694800 1.044765000 .30159200

H $2.50118900-0.29856700-1.17407700$

H - 2.50098400 -0.29820500 1.17434800

H 2.20821200 0.41912000 1.81799100

H 1.342712001 .179470000 .46540000

H - 2.20850200 $0.41913800-1.81785200$

H - $1.342726001 .17959100-0.46545500$

H $3.75318200-2.05924000-0.48587900$ H $3.35025200-1.778065001 .10130100$

H -3.75383900 -2.05872700 0.48705700

H -3.34847700 -1.77934600 -1.09978900 H $4.68410300-0.931950000 .40051900$ H $-4.68340400-0.93233500-0.40174400$ $\mathrm{H}=-666.209130$ Hartree

$G=-666,274514$ Hartree

Structure 18

S $0.13923200-1.992200000 .88106000$

S $1.17449700-1.53430600-1.14105200$

O $-3.77796100-0.44549200-1.25866300$

O $1.888161001 .95560200-1.67730000$

O -3.80628200 1.427429000 .10858100

O 0.874137002 .149337000 .39516300

N -1.474670001.09769600 1.13009500

N 2.38781900 0.445301001 .62941100

C - 1.793338000 .078491000 .05706800

C 2.739353000 .624397000 .15424000

C $-1.63612700-1.374086000 .49868700$
C $2.80900900-0.71276100-0.59555000$

C -3.27416900 $0.36685400-0.41320700$

C $1.743732001 .66557900-0.45357100$

H - $1.136301000 .27886100-0.79592700$

H 3.739175001 .070796000 .12347900

H - $2.17181900-1.592123001 .43001800$

H - $2.02785500-2.01735400-0.28920300$

H $3.29840500-0.54574900-1.55975300$

H $3.38858700-1.45131300-0.03676200$

H -0.58876500 1.624604000 .88827800

H - 1.374786000 .678371002 .06016500

H 1.877813001 .309726001 .89657900

H 3.216334000 .304248002 .21733600

H -2.32046300 1.721315001 .11506900

H $1.73276900-0.344106001 .76581800$

$H=-666.215106$ Hartree

$G=-666,274707$ Hartree

CYSTEINE- $\mathrm{Ag}_{2}$

Structure 1

$\begin{array}{lll}\text { N }-3.52676100 & -1.55410200 & 0.88851700 \\ \text { C }-3.54306900 & -0.90412000 & -0.48655400 \\ \text { H }-3.68040200 & -1.70437100 & -1.22312600 \\ \text { C -4.68551300 } & 0.10754000 & -0.64444300 \\ \text { C -2.11665100 } & -0.30478900 & -0.71702900 \\ \text { H }-4.57210800 & 0.61629000 & -1.60142900 \\ \text { H -5.66112000 } & -0.38256500 & -0.60676200 \\ \text { S -4.74948200 } & 1.41321100 & 0.73680900 \\ \text { O -1.21010400 } & -0.79319200 & 0.09213900 \\ \text { H -3.62379900 } & 2.11384900 & 0.36658200 \\ \text { O -1.96151900 } & 0.54202000 & -1.64531700 \\ \text { Ag } 1.00893700 & -0.24398900 & 0.02977600 \\ \text { H -2.51531600 } & -1.73520000 & 1.07631400 \\ \text { H -3.87350300 } & -0.88412100 & 1.59407000 \\ \text { H -4.07822400 } & -2.41666900 & 0.93633300 \\ \text { Ag 3.58952900 } & 0.27117400 & 0.0758200 \\ \text { H= -625.288391 Hartree } & \\ \text { G= -625.348063 Hartree } & \\ \text { Structure 2 } & & \\ \text { N 3.54137200 } & -1.61711800 & -0.76788700 \\ \text { C 3.52558500 } & -0.87174600 & 0.55756000 \\ \text { H 3.62958100 } & -1.61907800 & 1.35224600 \\ \text { C 4.67737600 } & 0.13421300 & 0.68025000 \\ \text { C 2.10251100 } & -0.24095600 & 0.70345700\end{array}$


H $4.54285000 \quad 0.70912800 \quad 1.59621800$

$\begin{array}{lll}\text { H } 5.64646700 & -0.36944800 & 0.70594600\end{array}$

S $4.799409001 .33981100-0.78489500$

$\begin{array}{llll}\text { O } & 1.21340600 & -0.76724300 & -0.10132300\end{array}$

H $3.67647100 \quad 2.08069000-0.49297700$

$\begin{array}{llll}\text { O } & 1.93162400 & 0.66417900 & 1.57229900\end{array}$

H $2.53305900-1.79136500-0.97827400$

H $3.92742000-1.00668400-1.50626400$

H $4.07431000-2.49182700-0.73340700$

Ag $-1.01127700-0.23305300-0.05127600$

$\begin{array}{llll}\text { Ag } & -3.59730500 & 0.25575200 & -0.06415800\end{array}$

$\mathrm{H}=-625.288410$ Hartree

$G=-625.347169$ Hartree

Structure 3

$\begin{array}{lll}\text { N } 4.49064200 & -1.42364800 & -0.44172700 \\ \text { C } 3.60759900 & -0.76319800 & 0.60577400 \\ \text { H } 3.66370100 & -1.37373300 & 1.51365700 \\ \text { C } 4.06404800 & 0.66135600 & 0.94666200 \\ \text { C } 2.14349800 & -0.86823100 & 0.06630200 \\ \text { H } 3.33681800 & 1.10791900 & 1.62392000 \\ \text { H } 5.04892800 & 0.66157400 & 1.41927100 \\ \text { S } 4.25870500 & 1.78867900 & -0.57191500 \\ \text { O } 1.95905800 & -1.68285500 & -0.90788100 \\ \text { H } 2.91928900 & 1.91979700 & -0.86181500 \\ \text { O } 1.27160100 & -0.14292800 & 0.68008500 \\ \text { H } 3.83424400 & -2.01770900 & -0.99793300 \\ \text { H } 4.88822400 & -0.70686800 & -1.06964600 \\ \text { H } 5.24895800 & -1.98165800 & -0.03666000 \\ \text { Ag }-0.97253400 & -0.07314500 & 0.21829500 \\ \text { Ag }-3.56470200 & 0.16174200 & -0.15953200 \\ \text { H= }-625.288363 & \text { Hartree } & \\ \text { G= -625.346991 Hartree } & \end{array}$

\section{Structure 4}

N $4.22280000 \quad-1.65554500 \quad 0.04682500$

$\begin{array}{lll}\text { C } 3.44981000 & -0.40540800 & 0.43900700\end{array}$

H $3.58371400-0.230823001 .50807900$

C $3.91984000 \quad 0.82012100-0.36877300$

$\begin{array}{lll}\text { C } 1.94790200 & -0.70209500 & 0.12763200\end{array}$

H $3.717343000 .69926300-1.43650500$

H $3.396462001 .70435000-0.00585800$

S $5.793246001 .13058600-0.27903200$

$\begin{array}{llll}\text { O } & 1.10817200 & 0.14630400 & 0.61719700\end{array}$

H 5.857409001 .450372001 .05833000
O $1.71090000-1.72866600-0.60450500$

H $3.60260200-2.15611000-0.62744000$

H $5.13772600-1.41832300-0.36360200$

$\begin{array}{llll}\text { H } 4.38176100 & -2.26998300 & 0.85359800\end{array}$

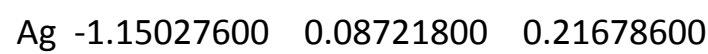

Ag $-3.75156300 \quad 0.12775800 \quad-0.17718200$

$\mathrm{H}=-625.287378$ Hartree

$\mathrm{G}=-625.346653$ Hartree

Structure 5

N $3.47791200 \quad-1.47449200 \quad 0.59126500$

C $3.45000200 \quad 0.04553900 \quad 0.64067400$

H $3.90559000 \quad 0.37160100 \quad 1.57766900$

$\begin{array}{llll}C & 4.20000100 & 0.65635100 & -0.55966900\end{array}$

$\begin{array}{llll}\text { C } 1.95144400 & 0.48193100 & 0.60359700\end{array}$

H $3.71464400 \quad 0.40771500-1.50748700$

H $4.224123001 .74026300-0.44718600$

S $5.97928500 \quad 0.01719600-0.76016700$

$\begin{array}{llll}\text { O } & 1.67797700 & 1.68237100 & 0.90054900\end{array}$

H $6.50162400 \quad 0.57753300 \quad 0.38368100$

$\begin{array}{llll}\text { O } & 1.13242200 & -0.46610800 & 0.22321100\end{array}$

H $2.58002100-1.74877900 \quad 0.13821800$

H $4.30161200-1.81654800 \quad 0.07440000$

H $3.49545500-1.880789001 .53372900$

Ag -1.12897700 $-0.15486000 \quad 0.04284300$

Ag $-3.73972200 \quad 0.06044100 \quad-0.18809600$

$\mathrm{H}=-625.287134$ Hartree

$G=-625.345770$ Hartree

Structure 6

N $4.46411200 \quad-1.45035300-0.43323700$

$\begin{array}{lll}\text { C } 3.59144300 & -0.77277600 & 0.61052500\end{array}$

H $3.63366600-1.38056300 \quad 1.52093400$

$\begin{array}{lll}\text { C } 4.06373600 & 0.64584600 & 0.95231000\end{array}$

$\begin{array}{llll}\text { C } 2.12605400 & -0.85937300 & 0.06964200\end{array}$

H $3.32372100 \quad 1.10369700 \quad 1.60750000$

H $5.03553200 \quad 0.635812001 .44836600$

S $4.209121001 .75052800-0.60070100$

O $1.93484200-1.66803500-0.90884400$

H $5.559600001 .99670500-0.52102500$

$\begin{array}{llll}\text { O } & 1.25890500 & -0.13454000 & 0.68984200\end{array}$

H $3.79599600-2.03352400-0.98834300$

H $4.87164300-0.74202600-1.06400700$

H $5.21325900-2.01973900-0.02724000$

Ag $-0.98334100 \quad-0.05761800 \quad 0.22199000$ 
Ag -3.57550000 $0.16234300-0.16614200$

$H=-625.286355$ Hartree

$\mathrm{G}=-625.345652$ Hartree

Structure 7
$\begin{array}{lll}\text { N } 3.51972200 & 1.50756000 & 0.94587100\end{array}$
C $3.54009100 \quad 0.92006000-0.45582900$
H $3.684749001 .74969900-1.15715700$
C $4.67407000-0.09192300-0.66058500$
C $2.11034000 \quad 0.34274200-0.72102000$
H $4.54200700-0.56137200-1.63492900$
H $5.65314000 \quad 0.38772000-0.61557100$
S $4.64115600-1.48683300 \quad 0.64565200$
O $1.20714200 \quad 0.78413200 \quad 0.11929700$
H $5.84977400-1.20879700 \quad 1.24030800$
$\begin{array}{llll}\text { O } & 1.94789600 & -0.43778200 & -1.70378900\end{array}$
H $2.50650900 \quad 1.679819001 .13642700$
H $3.85843800 \quad 0.80534100 \quad 1.62271000$
H $4.07264500 \quad 2.36591400 \quad 1.03524200$
Ag - $1.00994200 \quad 0.23785100 \quad 0.03683100$
Ag $-3.59114500 \quad-0.27568100 \quad 0.07214700$
$\mathrm{H}=-625.286308$ Hartree
$G=-625.345177$ Hartree

Structure 8

$\begin{array}{ccc}\text { N } 4.22226300 & -1.66168800 & 0.04358000 \\ \text { C } 3.45021100 & -0.40903200 & 0.43096300 \\ \text { H } 3.57495300 & -0.23512400 & 1.50136500 \\ \text { C } 3.92497500 & 0.81155800 & -0.37655000 \\ \text { C } 1.94813600 & -0.70365800 & 0.11563000 \\ \text { H } 3.77075900 & 0.66536300 & -1.44805400 \\ \text { H } 3.36721900 & 1.68740400 & -0.04715700 \\ \text { S } 5.78797600 & 1.14016200 & -0.09321700 \\ \text { O } 1.10983100 & 0.15588800 & 0.58677400 \\ \text { H } 6.12176500 & 1.38485400 & -1.40415100 \\ \text { O } 1.71098500 & -1.74340800 & -0.59765000 \\ \text { H } 4.39700900 & -2.26488500 & 0.85544200 \\ \text { H } 3.59130700 & -2.17205300 & -0.61414400 \\ \text { H } 5.12894300 & -1.42760400 & -0.38533600 \\ \text { Ag }-1.15098400 & 0.09284200 & 0.20355200 \\ \text { Ag }-3.75586500 & 0.12541800 & -0.16535600 \\ \text { H= -625.285431 Hartree } & \\ \text { G= -625.345191 Hartree } & \end{array}$

Structure 9

\begin{abstract}
N -4.18271900 $-1.76702100 \quad 0.10656800$
C -3.47445300 $-0.48781400-0.32967200$

H -3.65718200 $-0.36404300-1.40096500$

$\begin{array}{lll}\text { C }-3.94191600 & 0.75929900 & 0.42659800\end{array}$

$\begin{array}{lll}\text { C }-1.94362800 & -0.77344600 & -0.13887600\end{array}$

H -3.87461900 $0.63583000 \quad 1.51070200$

H-3.29593400 $1.58782300 \quad 0.13451600$

S -5.70455300 $1.32714800-0.01692600$

$\begin{array}{llll}0 & -1.15959400 & 0.23243900 & -0.31653900\end{array}$

H $-6.39338600 \quad 0.60221600 \quad 0.92871700$

$\begin{array}{llll}0 & -1.63095700 & -1.98580500 & 0.15031500\end{array}$

H-3.39470100 $-2.46043900 \quad 0.18385300$

H -4.62942600 -1.678104001 .02619600$

H-4.88525400 $-2.08268000-0.56950600$

Ag $1.12697700 \quad 0.13311100 \quad-0.11809000$

$\begin{array}{lll}\text { Ag } 3.74891200 & 0.12078000 & 0.10304300\end{array}$

$\mathrm{H}=-625.284898$ Hartree

$G=-625.345041$ Hartree
\end{abstract}

Structure 10

$\mathrm{N}-4.40701900 \quad-1.93625700 \quad 0.06813700$

C -3.71670300 $-0.61360000-0.27972700$

H -3.93127200 $-0.40148400-1.33129600$

C-4.24327900 $0.53368600 \quad 0.60314000$

C - $2.18487100-0.90341400-0.13308800$

H $-5.33664700 \quad 0.53696500 \quad 0.61140100$

$\begin{array}{lll}H-3.87508800 & 0.44580200 & 1.62858800\end{array}$

S -3.77601100 $2.24214800-0.07014200$

$\begin{array}{llll}0 & -1.39915500 & 0.11893400 & -0.21621400\end{array}$

H-2.42832000 $1.95372000-0.16015600$

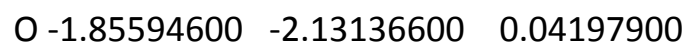

H -5.10239500 $-2.21036500-0.63348800$

H-3.61372100 $-2.62590300 \quad 0.09891200$

H -4.86406200 $-1.90981400 \quad 0.98720000$

$\begin{array}{lll}\text { Ag } 0.89277200 & 0.02031900 & -0.08417700\end{array}$

$\begin{array}{lll}\text { Ag } 3.51843600 & 0.06244500 & 0.07771000\end{array}$

$\mathrm{H}=-625.284728$ Hartree

$G=-625.344858$ Hartree

Structure 11

N $3.26157300 \quad 2.23371100 \quad 0.09649600$

C $3.52484600 \quad 0.76885500-0.26232400$

H $3.86618800 \quad 0.75519200-1.30244800$

$\begin{array}{llll}\text { C } 4.60322000 & 0.16026000 & 0.65085300\end{array}$

$\begin{array}{llll}\text { C } 2.13122800 & 0.05826300 & -0.19836400\end{array}$ 


$$
\begin{array}{ccc}
\text { H } 5.47472500 & 0.81819900 & 0.70842700 \\
\text { H } 4.21681500 & -0.01548500 & 1.65783600 \\
\text { S } 5.28968200 & -1.46951000 & -0.03013100 \\
\text { O } 2.08627400 & -1.20961000 & -0.16663600 \\
\text { H } 4.05393200 & -2.07926200 & -0.11489300 \\
\text { O } 1.12067400 & 0.88984800 & -0.20626900 \\
\text { H } 3.81459700 & 2.88473900 & -0.47033200 \\
\text { H } 2.23453600 & 2.35012600 & -0.07734200 \\
\text { H } 3.44439400 & 2.42395100 & 1.08935600 \\
\text { Ag }-1.07920200 & 0.25828000 & -0.09549200 \\
\text { Ag }-3.63957400 & -0.31418300 & 0.09885900 \\
\text { H= -625.284681 Hartree } & \\
\text { G= -625.344243 Hartree } &
\end{array}
$$

Structure 12

$$
\begin{array}{ccc}
\text { N } 3.47328300 & -1.48673400 & 0.55974500 \\
\text { C } 3.44805700 & 0.03236300 & 0.63669400 \\
\text { H } 3.89473000 & 0.34069800 & 1.58402200 \\
\text { C } 4.20406500 & 0.66349700 & -0.54681100 \\
\text { C } 1.95049800 & 0.47362900 & 0.59866800 \\
\text { H } 3.74417600 & 0.40043800 & -1.50202800 \\
\text { H } 4.19509000 & 1.74671000 & -0.42903300 \\
\text { S } 6.02612500 & 0.08299400 & -0.59258600 \\
\text { O } 1.67943800 & 1.67248100 & 0.90320000 \\
\text { H } 6.08111500 & -0.22099200 & -1.93218200 \\
\text { O } 1.13000400 & -0.47055800 & 0.21209900 \\
\text { H } 3.49802500 & -1.90992200 & 1.49467000 \\
\text { H } 2.57051700 & -1.75030800 & 0.10932200 \\
\text { H } 4.29180700 & -1.82063300 & 0.03020000 \\
\text { Ag }-1.13171600 & -0.15545200 & 0.03938900 \\
\text { Ag }-3.74270400 & 0.06313200 & -0.18503800 \\
\text { H= -625.285133 Hartree } & \\
\text { G= -625.344221 Hartree } &
\end{array}
$$

\section{CYSTEINE-Ag 4}

Structure 1

$$
\begin{array}{lrr}
\text { N }-4.36490300 & -1.72422500 & -1.51315500 \\
\text { C-4.92420500 } & -0.44787600 & -0.90494900 \\
\text { H -5.36512000 } & 0.13586500 & -1.72097800 \\
\text { C -6.01115100 } & -0.72798700 & 0.14081500 \\
\text { C-3.70593700 } & 0.36516100 & -0.36070100 \\
\text { H -6.30155200 } & 0.21360900 & 0.60601500 \\
\text { H -6.88873700 } & -1.19547900 & -0.31153300
\end{array}
$$

$\begin{array}{lll}\text { S -5.46628000 } & -1.93878500 & 1.50166300 \\ \text { O -2.55479200 } & -0.02852200 & -0.84704900 \\ \text { H-4.59981400 } & -1.08969300 & 2.15218100 \\ \text { O -3.92555600 } & 1.31487700 & 0.44693800 \\ \text { H-3.36992900 } & -1.49757700 & -1.73187400 \\ \text { H-4.37704700 } & -2.47872300 & -0.80781200 \\ \text { H -4.87274000 } & -2.03026100 & -2.34942500 \\ \text { Ag -0.58985200 } & 0.98148700 & -0.30895800 \\ \text { Ag 2.00365800 } & -0.14968500 & 0.04431600 \\ \text { Ag 1.47080800 } & 2.59576100 & 0.26066200 \\ \text { Ag 3.35964700 } & -2.45722700 & 0.01842200 \\ \text { H= -916.891589 Hartree } & \\ \text { G= -916.966823 Hartree } & \end{array}$

Structure 2

$\begin{array}{lll}\text { N } 4.48921300 & -1.31236000 & 1.39225500 \\ \text { C } 4.91358700 & -0.18304000 & 0.46575300 \\ \text { H } 5.64321100 & 0.43906400 & 0.98784000 \\ \text { C } 5.51636800 & -0.73296800 & -0.84244800 \\ \text { C } 3.63856200 & 0.65756200 & 0.14632200 \\ \text { H } 4.77602900 & -1.28933000 & -1.42371000 \\ \text { H } 5.88458200 & 0.10031000 & -1.44085400 \\ \text { S } 6.93241600 & -1.97283700 & -0.57422400 \\ \text { O } 3.79577000 & 1.79940300 & -0.37768600 \\ \text { H } 7.84537100 & -1.09122100 & -0.04080900 \\ \text { O } 2.51600600 & 0.04874500 & 0.43667200 \\ \text { H } 3.47662300 & -1.46142200 & 1.19919300 \\ \text { H } 5.05034300 & -2.16141000 & 1.22656300 \\ \text { H } 4.58733800 & -1.04598600 & 2.37866900 \\ \text { Ag } 0.47848500 & 1.01051200 & 0.13145000 \\ \text { Ag }-2.08393700 & -0.23959100 & -0.03928500 \\ \text { Ag }-1.71061400 & 2.53713200 & -0.09782600 \\ \text { Ag }-3.37567800 & -2.58406500 & -0.04826900 \\ \text { H= }-916.891589 & \text { Hartree } & \\ \text { G= }-916.966823 \text { Hartree } & \end{array}$

Structure 3

$\begin{array}{ccc}\text { N }-4.92325700 & 1.59018900 & 1.28099400 \\ \text { C-4.59459700 } & 0.27132000 & 0.59873100 \\ \text { H-5.02531700 } & -0.54259800 & 1.18456100 \\ \text { C-5.13293200 } & 0.24180800 & -0.84515400 \\ \text { C-3.03824000 } & 0.15626400 & 0.58786100 \\ \text { H-4.65492300 } & 1.00419700 & -1.46660400 \\ \text { H-4.93905400 } & -0.74055100 & -1.27514400 \\ \text { S -6.99040700 } & 0.62109200 & -0.98291300\end{array}$




$$
\begin{array}{lll}
\text { O-2.57589500 } & -1.00845100 & 0.27449900 \\
\text { H-7.45227000 } & -0.50679600 & -0.34292700 \\
\text { O-2.38348900 } & 1.22564600 & 0.85488100 \\
\text { H-4.06468500 } & 2.17345800 & 1.17931100 \\
\text { H-5.75099200 } & 2.03515900 & 0.85798700 \\
\text { H-5.10422100 } & 1.46166300 & 2.28317700 \\
\text { Ag -0.33481100 } & -1.41177900 & 0.11974600 \\
\text { Ag 1.92939700 } & 0.32037000 & -0.05356000 \\
\text { Ag 2.11603000 } & -2.47720300 & -0.07789600 \\
\text { Ag 2.66322400 } & 2.89399000 & -0.13178700 \\
\text { H= -916.891821 Hartree } & \\
\text { G=-916.966708 Hartree } &
\end{array}
$$

Structure 4

$$
\begin{array}{ccc}
\text { N } 5.28774200 & 0.49937800 & -1.01028100 \\
\text { C } 4.69800800 & -0.90162100 & -0.99411000 \\
\text { H } 4.77607700 & -1.30263700 & -2.01092100 \\
\text { C } 5.44223400 & -1.84442400 & -0.04043100 \\
\text { C } 3.17584800 & -0.74767500 & -0.67391900 \\
\text { H } 4.90189200 & -2.78966800 & -0.00143500 \\
\text { H } 6.46713700 & -2.02279800 & -0.36973000 \\
\text { S } 5.50572700 & -1.15794500 & 1.74247700 \\
\text { O } 2.73146200 & 0.47662600 & -0.82273400 \\
\text { H } 6.87151200 & -1.03006300 & 1.83963000 \\
\text { O } 2.52092400 & -1.77854400 & -0.34312800 \\
\text { H } 4.47139800 & 1.12487400 & -1.18945400 \\
\text { H } 5.66524900 & 0.72828600 & -0.07683700 \\
\text { H } 6.02124500 & 0.62200000 & -1.71572600 \\
\text { Ag } 0.60719700 & 1.09764800 & -0.30720300 \\
\text { Ag }-1.94047600 & -0.14046800 & 0.04547000 \\
\text { Ag }-1.51497200 & 2.60865500 & 0.32844500 \\
\text { Ag }-3.24102500 & -2.47904400 & -0.01789300 \\
\text { H= -916.890752 Hartree } & \\
\text { G= -916.966475 Hartree } &
\end{array}
$$

Structure 5

$$
\begin{array}{ccc}
\text { N }-5.49646800 & -0.04568200 & 1.53066400 \\
\text { C -4.75478100 } & -0.21267900 & 0.21348000 \\
\text { H -5.19518900 } & -1.04798600 & -0.33397300 \\
\text { C -4.81035300 } & 1.07791800 & -0.62481600 \\
\text { C-3.27310300 } & -0.54150600 & 0.57915100 \\
\text { H -4.32230600 } & 1.90838400 & -0.10966600 \\
\text { H -4.30888300 } & 0.89947500 & -1.57528200 \\
\text { S -6.61579600 } & 1.59270600 & -0.99086700 \\
\text { O -2.54489100 } & -0.95799900 & -0.40269700
\end{array}
$$

$$
\begin{array}{lll}
\text { H }-6.49902700 & 2.91526700 & -0.63401300 \\
\text { O-2.92587600 } & -0.33531900 & 1.79569600 \\
\text { H -5.96456400 } & -0.91412500 & 1.81389400 \\
\text { H-4.75742000 } & 0.17064300 & 2.23460500 \\
\text { H-6.20653200 } & 0.69837900 & 1.46879500 \\
\text { Ag -0.31250100 } & -1.37163900 & -0.19660000 \\
\text { Ag 2.15070300 } & -2.41639500 & -0.08141700 \\
\text { Ag 1.93911100 } & 0.37901400 & -0.04706000 \\
\text { Ag 2.65625000 } & 2.95392500 & 0.11495000 \\
\text { H= -916.889730 Hartree } & \\
\text { G= -916.964973 Hartree } &
\end{array}
$$

\section{Structure 6}

$$
\begin{array}{ccc}
\text { N-4.68372400 } & -0.29291600 & 2.08123800 \\
\text { C-4.80455800 } & 0.00959300 & 0.58511100 \\
\text { H-5.45183700 } & -0.76378100 & 0.15930300 \\
\text { C-5.42269700 } & 1.39855500 & 0.34998600 \\
\text { C-3.36999100 } & -0.17903500 & -0.00808200 \\
\text { H-6.31754400 } & 1.52863100 & 0.96478200 \\
\text { H-4.70990500 } & 2.19482000 & 0.57858000 \\
\text { S-6.03835200 } & 1.62483000 & -1.42734200 \\
\text { O-3.10935900 } & 0.28744500 & -1.15832500 \\
\text { H-4.81643900 } & 1.34847100 & -2.00550800 \\
\text { O-2.56431100 } & -0.84970200 & 0.77726900 \\
\text { H-5.48925700 } & -0.81440800 & 2.44282900 \\
\text { H-3.80254300 } & -0.85391300 & 2.15350700 \\
\text { H-4.56905000 } & 0.56287200 & 2.63823300 \\
\text { Ag -0.40792400 } & -1.32254000 & 0.22170100 \\
\text { Ag } 1.93827200 & -2.47360700 & -0.36456100 \\
\text { Ag } 1.91861200 & 0.31130500 & -0.03696400 \\
\text { Ag 2.75378600 } & 2.84598700 & 0.15479100 \\
\text { H=-916.888923 Hartree } & \\
\text { G=-916.964699 Hartree } &
\end{array}
$$

\section{Structure 7}

$\begin{array}{lll}\text { N } 5.15789500 & -1.09593600 & 0.30909300 \\ \text { C } 4.60208800 & 0.28151700 & 0.63633400 \\ \text { H } 4.97658400 & 0.58440300 & 1.61665800 \\ \text { C } 5.00420600 & 1.30930900 & -0.43737200 \\ \text { C } 3.04798100 & 0.15774500 & 0.68719200 \\ \text { H } 4.60953800 & 1.03708800 & -1.41906800 \\ \text { H } 4.61642700 & 2.28617300 & -0.14964300 \\ \text { S } 6.90606800 & 1.44116100 & -0.58644100 \\ \text { O } 2.39362600 & 1.11032200 & 1.20433900 \\ \text { H } 6.98045900 & 1.36293200 & -1.95671400\end{array}$




$$
\begin{array}{lll}
\text { O } 2.58468300 & -0.93858500 & 0.14003800 \\
\text { H } 5.36269800 & -1.63041900 & 1.16134600 \\
\text { H } 4.39584600 & -1.58794000 & -0.20179200 \\
\text { H } 6.02320700 & -1.02506800 & -0.24652500 \\
\text { Ag } 0.34929400 & -1.35843700 & 0.03321400 \\
\text { Ag }-1.94261700 & 0.32885600 & -0.04482600 \\
\text { Ag }-2.08235700 & -2.47031100 & -0.14361500 \\
\text { Ag -2.69281400 } & 2.89820000 & -0.00768200 \\
\text { H= -916.889533 Hartree } & \\
\text { G= -916.964496 Hartree } &
\end{array}
$$

\section{Structure 8}

$$
\begin{array}{lrl}
\text { N }-4.83161000 & -0.54941000 & 1.39916800 \\
\text { C-3.44382800 } & -0.10484100 & 0.96565600 \\
\text { H-2.92046300 } & 0.27160400 & 1.85140700 \\
\text { C-2.62468900 } & -1.26190100 & 0.37855200 \\
\text { C-3.66502600 } & 1.11469500 & 0.00455600 \\
\text { H-1.66589500 } & -0.88379200 & 0.02259300 \\
\text { H-2.44267100 } & -2.03368400 & 1.12865100 \\
\text { S-3.51891500 } & -2.08329800 & -1.09888200 \\
\text { O-4.86407900 } & 1.57101100 & -0.04421700 \\
\text { H-3.50405300 } & -3.37306000 & -0.62325100 \\
\text { O -2.63746200 } & 1.57005300 & -0.62599000 \\
\text { H-5.44873200 } & 0.25735900 & 1.14065000 \\
\text { H-5.12837500 } & -1.36862900 & 0.84596000 \\
\text { H-4.89356400 } & -0.76697700 & 2.39850600 \\
\text { Ag -0.38432500 } & 1.66599200 & -0.25488000 \\
\text { Ag 1.52405000 } & -0.42243000 & 0.01092600 \\
\text { Ag 2.18335400 } & 2.30974700 & 0.13152500 \\
\text { Ag 1.66716000 } & -3.09672300 & 0.07609900 \\
\text { H=-916.889768 Hartree } & \\
\text { G=-916.964344 Hartree } &
\end{array}
$$

Structure 9
N $4.92293800 \quad-0.51834400 \quad 1.35321900$
$\begin{array}{lll}\text { C } 4.57330300 & 0.55087900 & 0.32145700\end{array}$
H $4.484815001 .49726900 \quad 0.86432700$
C $5.61285200 \quad 0.69022400-0.79378500$
C $3.14659600 \quad 0.19573700-0.21900200$
H $5.82007400-0.26050000-1.29135600$
H $5.214271001 .38351800-1.53534500$
S $7.260579001 .45975700-0.23169200$
O $2.71906400 \quad 0.79941800 \quad-1.24469300$
H $7.90904300 \quad 0.30072900 \quad 0.12928500$
$\begin{array}{llll}\text { O } & 2.52017300 & -0.69728600 & 0.50955100\end{array}$
H $3.99280900-0.93590700 \quad 1.59699500$
H $5.51111000-1.262041000 .95864200$
H $5.38908800 \quad-0.13457600 \quad 2.18195000$
Ag $0.34674200 \quad-1.25005500 \quad 0.12202200$
Ag -2.03623500 $0.29876600 \quad-0.01951300$
Ag -2.00388400 - $-2.50243000-0.17690200$
Ag $-2.92069700 \quad 2.82064900 \quad 0.10331100$
$\mathrm{H}=-916.889149$ Hartree
$G=-916.963868$ Hartree

Structure 10

$\begin{array}{lcc}\text { N-5.61272500 } & -0.43300800 & 1.72074000 \\ \text { C-4.70510300 } & -0.77871700 & 0.54298600 \\ \text { H-4.42574800 } & -1.83128600 & 0.64182800 \\ \text { C-5.35999800 } & -0.54047400 & -0.82177900 \\ \text { C-3.41003900 } & 0.08089000 & 0.74457800 \\ \text { H-5.74616200 } & 0.47714400 & -0.92403900 \\ \text { H-4.60113400 } & -0.69917400 & -1.58864700 \\ \text { S-6.75019000 } & -1.76945500 & -1.24597700 \\ \text { O-2.53979700 } & 0.00637000 & -0.20296000 \\ \text { H-7.79711300 } & -1.09115900 & -0.66491700 \\ \text { O -3.33992300 } & 0.75109700 & 1.83760500 \\ \text { H-4.99428600 } & 0.15619900 & 2.33565000 \\ \text { H-6.43068700 } & 0.12178300 & 1.44457000 \\ \text { H-5.93801700 } & -1.26412800 & 2.22463200 \\ \text { Ag -0.48575500 } & 0.99715100 & -0.12893700 \\ \text { Ag 2.10101000 } & -0.21587900 & -0.03842600 \\ \text { Ag 1.69110800 } & 2.55504100 & -0.17923300 \\ \text { Ag 3.39818800 } & -2.55241800 & 0.10296800 \\ \text { H= -916.889136 Hartree } & \\ \text { G=-916.963807 Hartree } & \end{array}$

Structure 11

$\begin{array}{llll}\text { N } & 4.42265100 & -3.11062700 & -0.62676100 \\ \text { C } 4.40457700 & -1.75424500 & 0.08523600 \\ \text { H } & 4.54110800 & -1.94885000 & 1.15318700 \\ \text { C } & 5.53459000 & -0.84346300 & -0.42911400 \\ \text { C } 2.96067500 & -1.19500500 & -0.13549400 \\ \text { H } & 6.48300700 & -1.38697700 & -0.45101700 \\ \text { H } & 5.31367100 & -0.46214400 & -1.42931900 \\ \text { S } 5.86304000 & 0.62440400 & 0.72316800 \\ \text { O } 2.76122900 & 0.03229400 & 0.22127700 \\ \text { H } 4.55036200 & 1.05205900 & 0.72604100 \\ \text { O } & 2.10591600 & -2.01047300 & -0.63463200 \\ \text { H } 4.82877200 & -3.85200300 & -0.04640800\end{array}$ 
H $3.40916600-3.30113500-0.82486400$

H $4.93246500-3.07577100-1.51766700$ Ag $0.71972600 \quad 1.03919200 \quad 0.01787900$ Ag -1.37206400 $2.69802000 \quad-0.18549600$ Ag -1.91768200 $-0.04708100 \quad 0.03340200$ Ag -3.28449000 $-2.34246100 \quad 0.16378500$ $\mathrm{H}=-916.889139$ Hartree $\mathrm{G}=-916.963806$ Hartree

Structure 12

$\begin{array}{ccc}\text { N } 3.57457100 & 1.38756000 & -1.92476600 \\ \text { H } 3.01895100 & 1.53934700 & -2.77561300 \\ \text { C } 2.67693600 & 1.19366700 & -0.69585300 \\ \text { H } 1.76978600 & 0.67007100 & -1.00466300 \\ \text { C } 3.46903800 & 0.35151400 & 0.33041700 \\ \text { C } 2.36907400 & 2.64565300 & -0.20665000 \\ \text { H } 3.74354000 & -0.61026200 & -0.11559800 \\ \text { H } 4.37620400 & 0.87304200 & 0.64833000 \\ \text { S } 2.51766200 & -0.03329300 & 1.91712700 \\ \text { O } 1.21306600 & 2.89430800 & 0.30985600 \\ \text { H } 1.68156100 & -1.00952700 & 1.34194900 \\ \text { H } 4.20566900 & 0.59518400 & -2.08969000 \\ \text { H } 4.11331900 & 2.25631300 & -1.72040600 \\ \text { O } 3.32853700 & 3.48408000 & -0.37771000 \\ \text { Ag }-0.73642700 & 1.70175400 & 0.21493100 \\ \text { Ag }-3.13335600 & 0.53956900 & -0.10431400 \\ \text { Ag }-0.86221600 & -1.10678700 & -0.12952500 \\ \text { Ag } 0.99504800 & -3.04233400 & -0.14087200 \\ \text { H= -916.889563 Hartree } & \\ \text { G= -916.963074 Hartree } & \end{array}$

CYSTEINE-Au

Structure 1

$\begin{array}{ccc}\text { N }-4.11469500 & -1.50649100 & 0.85439400 \\ \text { C }-4.09580200 & -0.83139200 & -0.50868400 \\ \text { H }-4.23357000 & -1.61693600 & -1.26094800 \\ \text { C -5.22460100 } & 0.19816400 & -0.66301200 \\ \text { C - } 2.67131100 & -0.24070000 & -0.72154100 \\ \text { H -5.08740900 } & 0.72990800 & -1.60421100 \\ \text { H -6.20410500 } & -0.28484900 & -0.65919800 \\ \text { S -5.30440200 } & 1.46541300 & 0.75148100 \\ \text { O - } 1.76954900 & -0.78893000 & 0.07111100 \\ \text { H -4.17731500 } & 2.17916000 & 0.41222000\end{array}$

O -2.49001400 $0.63899700-1.60357600$

Au $0.33333000 \quad-0.28001100 \quad 0.01872300$ H -3.12988800 $-1.77292300 \quad 1.05172600$ H -4.41658400 $-0.82477600 \quad 1.57066800$ $\begin{array}{lll}\mathrm{H}-4.73214700 & -2.32484800 & 0.88652100\end{array}$ $\begin{array}{lll}\text { Au } 2.85250700 & 0.24782200 & 0.04732600\end{array}$ $\mathrm{H}=-604.681238$ Hartree $\mathrm{G}=-604.739444$ Hartree

\section{Structure 2}

$\begin{array}{lll}\text { N } 5.02758300 & -1.42516400 & -0.48720000 \\ \text { C } 4.13877300 & -0.76548900 & 0.55551600 \\ \text { H } 4.18837100 & -1.37838800 & 1.46207900 \\ \text { C } 4.60462900 & 0.65621300 & 0.90091100 \\ \text { C } 2.68333000 & -0.86378100 & 0.01228000 \\ \text { H } 3.88317400 & 1.10540100 & 1.58228300 \\ \text { H } 5.58979300 & 0.64364100 & 1.37241400 \\ \text { S } 4.80695600 & 1.78566600 & -0.61385600 \\ \text { O } 2.46540600 & -1.66890800 & -0.94843100 \\ \text { H } 3.46794400 & 1.94533100 & -0.89072000 \\ \text { O } 1.82789100 & -0.11158800 & 0.64281300 \\ \text { H } 4.40673000 & -2.05873900 & -1.03143900 \\ \text { H } 5.39798800 & -0.70825100 & -1.13264900 \\ \text { H } 5.80864400 & -1.94479800 & -0.07261200 \\ \text { Au }-0.29735300 & -0.05725000 & 0.22333800 \\ \text { Au }-2.83877100 & 0.10641000 & -0.15276200 \\ \text { H= }-604.681093 \text { Hartree } & \\ \text { G= -604.739340 Hartree } & \end{array}$

Structure 3

$\begin{array}{ccc}\text { N } 4.07834700 & -1.44695300 & 0.59229200 \\ \text { C } 4.04717000 & 0.07344500 & 0.62532000 \\ \text { H } 4.53275900 & 0.40692300 & 1.54469300 \\ \text { C } 4.76803000 & 0.66999400 & -0.60224700 \\ \text { C } 2.56128800 & 0.52683300 & 0.63196300 \\ \text { H } 4.26061000 & 0.40840400 & -1.53472300 \\ \text { H } 4.79452200 & 1.75526200 & -0.50408500 \\ \text { S } 6.54099800 & 0.02650800 & -0.82794500 \\ \text { O } 2.28799600 & 1.71509400 & 0.94566500 \\ \text { H } 7.08811400 & 0.61410500 & 0.29019100 \\ \text { O } 1.72920700 & -0.42415000 & 0.25107100 \\ \text { H } 3.20765600 & -1.74973000 & 0.11568100 \\ \text { H } 4.92255400 & -1.78456000 & 0.10406300 \\ \text { H } 4.07284000 & -1.84199400 & 1.54006900 \\ \text { Au }-0.40857300 & -0.14083000 & 0.08105000\end{array}$


Au -2.96459200 $0.06420900 \quad-0.15647900$ $\mathrm{H}=-604.679987$ Hartree

$\mathrm{G}=-604.737925$ Hartree

Structure 4

$\begin{array}{ccc}\text { N } 4.84571900 & -1.65142100 & 0.05477800 \\ \text { C } 4.04058900 & -0.40772300 & 0.40064100 \\ \text { H } 4.15081300 & -0.20905900 & 1.46808300 \\ \text { C } 4.50983800 & 0.81110900 & -0.42128000 \\ \text { C } 2.55687600 & -0.73335600 & 0.07100800 \\ \text { H } 4.32865200 & 0.66964000 & -1.49000300 \\ \text { H } 3.96807000 & 1.69376400 & -0.08251100 \\ \text { S } 6.37592300 & 1.14372600 & -0.29845600 \\ \text { O } 1.71226800 & 0.13654400 & 0.54802900 \\ \text { H } 6.40595300 & 1.50805000 & 1.02856400 \\ \text { O } 2.31467500 & -1.76141100 & -0.63729900 \\ \text { H } 4.29503400 & -2.17061000 & -0.65753400 \\ \text { H } 5.78213900 & -1.39783200 & -0.29565600 \\ \text { H } 4.96273900 & -2.26262300 & 0.87195500 \\ \text { Au }-0.42469200 & 0.05195100 & 0.19629500 \\ \text { Au }-2.97641600 & 0.07979200 & -0.14615700 \\ \text { H= -604.680169 Hartree } & \\ \text { G= -604.738243 Hartree } & \end{array}$

Structure 5

$\begin{array}{ccc}\text { N }-4.10772100 & -1.47616000 & 0.89625000 \\ \text { C-4.09639900 } & -0.84511100 & -0.48671400 \\ \text { H-4.24238000 } & -1.65186000 & -1.21434200 \\ \text { C-5.21733400 } & 0.18683600 & -0.67017100 \\ \text { C-2.66972600 } & -0.27007100 & -0.72858300 \\ \text { H-5.07617300 } & 0.68038700 & -1.63113800 \\ \text { H-6.20121200 } & -0.28369300 & -0.64345800 \\ \text { S-5.17074400 } & 1.54515500 & 0.67241600 \\ \text { O-1.76906300 } & -0.78633500 & 0.08752600 \\ \text { H-6.37472200 } & 1.25271600 & 1.26968600 \\ \text { O-2.48428600 } & 0.56328800 & -1.65294100 \\ \text { Au 0.33269200 } & -0.27941700 & 0.02273400 \\ \text { H-3.12265800 } & -1.74412100 & 1.09162200 \\ \text { H-4.39129300 } & -0.76873400 & 1.59390900 \\ \text { H -4.73303900 } & -2.28656600 & 0.96131000 \\ \text { Au 2.85154800 } & 0.25115100 & 0.04531800 \\ \text { H= -604.679203 Hartree } & \\ \text { G=-604.737544 Hartree } & \end{array}$

Structure 6
N $5.01540900-1.44096800-0.47577000$
C $4.12836700-0.77045900 \quad 0.56011400$
H $4.16338200-1.381438001 .46848400$
C $4.60070100 \quad 0.64756600 \quad 0.91016400$
C $2.67433600-0.85669100 \quad 0.00968600$
H 3.862333001 .102598001 .56879000
H $5.57187100 \quad 0.62971900 \quad 1.40691100$
S $4.749006001 .75816100-0.63717000$
O $2.45836300-1.64832300-0.96304600$
H $6.100289001 .99926000-0.55631500$
O $1.81699800-0.11696400 \quad 0.65156900$
H $4.38822000-2.06416400-1.02547700$
H $5.39755100-0.72826500-1.11882000$
H $5.78707500-1.97106000-0.05715800$
Au $-0.30571200 \quad-0.05636300 \quad 0.22521600$
Au -2.84600900 $0.11167200-0.15621900$
$\mathrm{H}=-604.679108$ Hartree
$G=-604.737437$ Hartree

$$
\begin{array}{lll}
\text { Structure 7 } & & \\
\text { N } 4.84414500 & -1.65458900 & 0.05154500 \\
\text { C } 4.04003800 & -0.41015400 & 0.39680400 \\
\text { H } 4.14319600 & -0.21475500 & 1.46562100 \\
\text { C } 4.51428500 & 0.80616600 & -0.42181100 \\
\text { C } 2.55612600 & -0.73218400 & 0.06354600 \\
\text { H } 4.37236400 & 0.64739500 & -1.49287100 \\
\text { H } 3.94594100 & 1.68080900 & -0.10780400 \\
\text { S } 6.36928300 & 1.15587600 & -0.12151400 \\
\text { O } 1.71290700 & 0.14316000 & 0.53236100 \\
\text { H } 6.73741000 & 1.28178400 & -1.43986900 \\
\text { O } 2.31327000 & -1.76571600 & -0.63641500 \\
\text { H } 4.96607800 & -2.26294200 & 0.87008700 \\
\text { H } 4.29030100 & -2.17624700 & -0.65655400 \\
\text { H } 5.77838800 & -1.40023600 & -0.30328100 \\
\text { Au }-0.42540100 & 0.05544800 & 0.18960600 \\
\text { Au }-2.97869100 & 0.07784100 & -0.14088100 \\
\text { H= -604.678180 Hartree } & \\
\text { G= -604.737121 Hartree } & \\
& & \\
\text { Structure } 8 & & \\
\text { N } 3.99954800 & -1.57795600 & -0.05971400 \\
\text { C } 4.03676700 & -0.11080400 & 0.35649000 \\
\text { H } 4.30803500 & -0.09576700 & 1.41732900 \\
\text { C } 5.04487300 & 0.71413700 & -0.44968500 \\
\text { C } 2.58184900 & 0.44028200 & 0.24599500 \\
& &
\end{array}
$$




$$
\begin{array}{ccc}
\text { H } 4.87353200 & 0.63900600 & -1.52642900 \\
\text { H } 4.93645500 & 1.75901500 & -0.15758500 \\
\text { S } 6.85888200 & 0.27232800 & -0.08182100 \\
\text { O } 2.38352800 & 1.68232800 & 0.26659200 \\
\text { H } 7.03483300 & -0.67820100 & -1.06158000 \\
\text { O } 1.67973600 & -0.52193100 & 0.17803500 \\
\text { H } 3.02597200 & -1.89012500 & 0.13636800 \\
\text { H } 4.16521700 & -1.69489800 & -1.06718600 \\
\text { H } 4.68164000 & -2.14676100 & 0.45355400 \\
\text { Au }-0.45191400 & -0.17136600 & 0.06440400 \\
\text { Au }-3.00696800 & 0.11125600 & -0.07631700 \\
\text { H= -604.677449 Hartree } & \\
\text { G= -604.736615 Hartree } &
\end{array}
$$

\section{Structure 9}

$$
\begin{array}{ccc}
\text { N } 4.08362800 & -1.43804400 & 0.60823100 \\
\text { C } 4.04759700 & 0.08177800 & 0.63639100 \\
\text { H } 4.54002400 & 0.42039500 & 1.55036200 \\
\text { C } 4.74840000 & 0.67668300 & -0.60227200 \\
\text { C } 2.56065500 & 0.53178700 & 0.65504800 \\
\text { H } 4.23589100 & 0.39702500 & -1.52523500 \\
\text { H } 4.75721000 & 1.76246200 & -0.51031900 \\
\text { S } 6.55911200 & 0.07999200 & -0.72984400 \\
\text { O } 2.28602800 & 1.71942600 & 0.96949600 \\
\text { H } 6.50287100 & -0.37246400 & -2.02675300 \\
\text { O } 1.72921900 & -0.42220900 & 0.28045500 \\
\text { Au }-0.40725800 & -0.14077200 & 0.09460700 \\
\text { H } 4.08310600 & -1.83001300 & 1.55728100 \\
\text { H } 3.21287700 & -1.74616100 & 0.13566800 \\
\text { H } 4.92819400 & -1.77226600 & 0.11851600 \\
\text { Au }-2.96050500 & 0.06239500 & -0.17073700 \\
\text { H= -604.677848 Hartree } & \\
\text { G= -604.736419 Hartree } &
\end{array}
$$

\section{Structure 10}

$$
\begin{array}{ccc}
\text { N }-4.81282300 & -1.74345500 & 0.11808500 \\
\text { C }-4.05866600 & -0.49071000 & -0.31682600 \\
\text { H }-4.22483900 & -0.37544500 & -1.39207600 \\
\text { C - } 4.51143900 & 0.77595800 & 0.41767000 \\
\text { C - } 2.54640300 & -0.81523100 & -0.11006500 \\
\text { H }-4.43184900 & 0.67749000 & 1.50309300 \\
\text { H -3.86784200 } & 1.59602500 & 0.09899300 \\
\text { S -6.28109000 } & 1.32263300 & -0.02070000 \\
\text { O - } 1.75387100 & 0.20636100 & -0.24938300 \\
\text { H - } 6.94572300 & 0.66992300 & 0.99235800
\end{array}
$$

$$
\begin{array}{lll}
\text { O -2.23427000 } & -2.02455000 & 0.13893300 \\
\text { H -4.09200700 } & -2.50004400 & 0.11921500 \\
\text { H -5.18464600 } & -1.66263400 & 1.07212200 \\
\text { H -5.58522000 } & -1.97592100 & -0.51520800 \\
\text { Au } 0.40623700 & 0.08307200 & -0.09010000 \\
\text { Au 2.97507300 } & 0.07310600 & 0.07193600 \\
\text { H= -604.677347 Hartree } & \\
\text { G= -604.736312 Hartree } &
\end{array}
$$

Structure 11

$$
\begin{array}{lll}
\text { N } 3.86366200 & 2.18079700 & 0.09619000 \\
\text { C } 4.08884600 & 0.70627000 & -0.25161400 \\
\text { H } 4.36232400 & 0.67754800 & -1.31165400 \\
\text { C } 5.22006700 & 0.10389100 & 0.59921400 \\
\text { C } 2.70856600 & 0.00030900 & -0.09552600 \\
\text { H } 6.08978800 & 0.76659400 & 0.60286500 \\
\text { H } 4.89510000 & -0.06987500 & 1.62760400 \\
\text { S } 5.88000600 & -1.52181300 & -0.11381200 \\
\text { O } 2.64558200 & -1.24939700 & 0.05693500 \\
\text { H } 4.67997800 & -2.19330600 & -0.02863200 \\
\text { O } 1.69943400 & 0.84733600 & -0.17221200 \\
\text { H } 4.47336600 & 2.80542600 & -0.44245400 \\
\text { H } 2.86075500 & 2.35908100 & -0.12069400 \\
\text { H } 4.00864700 & 2.36243200 & 1.09755900 \\
\text { Au }-0.38487900 & 0.27628300 & -0.06130100 \\
\text { Au }-2.89816700 & -0.26705200 & 0.05032400 \\
\text { H= }-604.677119 & \text { Hartree } & \\
\text { G= -604.735670 Hartree } &
\end{array}
$$

Structure 12

$$
\begin{array}{ccc}
\text { N }-4.91429700 & -1.94200600 & 0.15781800 \\
\text { C-4.22803500 } & -0.62953600 & -0.23303500 \\
\text { H-4.48744500 } & -0.43657700 & -1.27887800 \\
\text { C-4.71387500 } & 0.53292500 & 0.65191400 \\
\text { C-2.70067500 } & -0.93132800 & -0.15763600 \\
\text { H-5.80358300 } & 0.51325700 & 0.74028100 \\
\text { H-4.27134800 } & 0.48567500 & 1.64973600 \\
\text { S-4.34659100 } & 2.23102800 & -0.10034500 \\
\text { O-1.92825800 } & 0.11845500 & -0.16937700 \\
\text { H-2.98284500 } & 2.04776300 & -0.16455800 \\
\text { O-2.34703600 } & -2.15311700 & -0.11173500 \\
\text { H-5.74801600 } & -2.12801500 & -0.40970200 \\
\text { H-4.19193100 } & -2.68113500 & 0.00959200 \\
\text { H-5.18482600 } & -1.95300300 & 1.14927500 \\
\text { Au } 0.23803900 & 0.01602300 & -0.06850700
\end{array}
$$


Au $2.80846000 \quad 0.04087000 \quad 0.06200700$ $\mathrm{H}=-604.677088$ Hartree

$\mathrm{G}=-604.735869$ Hartree

CYSTEINE-Au

Structure 1

$\begin{array}{ccc}\text { N } 4.76805700 & 3.36224800 & -0.15855600 \\ \text { C } 4.65486700 & 2.00016200 & -0.82499900 \\ \text { H } 4.64412900 & 2.16430900 & -1.90822100 \\ \text { C } 5.84040500 & 1.08697100 & -0.47969400 \\ \text { C } 3.25975500 & 1.43833300 & -0.42787700 \\ \text { H } 5.66247200 & 0.10210300 & -0.90942200 \\ \text { H } 6.77488000 & 1.49149400 & -0.87495700 \\ \text { S 6.14551000 } & 0.90065600 & 1.38619200 \\ \text { O 2.41285500 } & 2.24853700 & 0.06433500 \\ \text { H 5.04780000 } & 0.11831000 & 1.66588500 \\ \text { O 3.10269000 } & 0.16814000 & -0.67804900 \\ \text { H 3.79301600 } & 3.67575600 & 0.02335700 \\ \text { H 5.24778600 } & 3.26432100 & 0.75168200 \\ \text { H 5.26983100 } & 4.04712900 & -0.73384900 \\ \text { Au } 1.24960100 & -0.87425000 & -0.28622400 \\ \text { Au -1.45570100 } & 0.00560900 & 0.03504800 \\ \text { Au -0.68446800 } & -2.60823200 & 0.12965100 \\ \text { Au -2.84106500 } & 2.21996400 & 0.07369600 \\ \text { H= -875.672270 Hartree } & \\ \text { G=-875.747697 Hartree } & \end{array}$

Structure 2

$\begin{array}{lll}\text { N } 5.25158700 & -1.29290500 & -1.69744100 \\ \text { C } 4.67418900 & -2.16240700 & -0.59133400 \\ \text { H } 4.30087900 & -3.07881000 & -1.06295100 \\ \text { C } 5.73195600 & -2.54339900 & 0.45452000 \\ \text { C } 3.44425200 & -1.41952800 & 0.00344700 \\ \text { H } 5.24530000 & -3.08279300 & 1.26663500 \\ \text { H } 6.51139600 & -3.17211300 & 0.01869100 \\ \text { S } 6.65844800 & -1.05048900 & 1.17916500 \\ \text { O } 2.97367100 & -0.48373500 & -0.80207900 \\ \text { H } 5.62462100 & -0.55959200 & 1.94414600 \\ \text { O } 2.99316500 & -1.76334600 & 1.12638900 \\ \text { H } 4.46436900 & -0.71687300 & -2.05263700 \\ \text { H } 5.96355600 & -0.65762400 & -1.30004900 \\ \text { H } 5.67425300 & -1.84275500 & -2.45335000 \\ \text { Au } 1.23194100 & 0.69277000 & -0.31517900\end{array}$

Au - $1.52496900 \quad 0.01141000 \quad 0.03324400$

Au $-0.55725800 \quad 2.55729500 \quad 0.17554400$ Au -3.09804000 $-2.07543100 \quad 0.04133600$ $\mathrm{H}=-875.672229$ Hartree $\mathrm{G}=-875.747429$ Hartree

Structure 3

N -4.40452600 $-2.73803500 \quad-1.04759300$ C -5.15621300 $-1.41940500-0.95157500$ H -5.49019800 $-1.16304800-1.96365000$ C $-6.38759600-1.52280000-0.04000100$ C - $4.14157500-0.32528900-0.51419400$ H -6.82722100 $-0.53214900 \quad 0.07217600$ $\begin{array}{lll}H-7.13147700 & -2.20448500 & -0.45805400\end{array}$ S $-6.00268300-2.22363900 \quad 1.68388500$ O $-2.88632500 \quad-0.68255500-0.71716600$ H -5.33967000 $-1.12213800 \quad 2.17591400$ $\begin{array}{llll}0 & -4.56369400 & 0.76859800 & -0.05747000\end{array}$ H -3.41317700 $-2.49124900-1.23367800$ $\begin{array}{lll}H-4.45152600 & -3.22725000 & -0.13800200\end{array}$ $\begin{array}{lll}H-4.77339200 & -3.35292400 & -1.78124800\end{array}$ Au -1.22426800 $0.62500500-0.28833200$ $\begin{array}{lll}\text { Au } 1.55378700 & 0.00674300 & 0.04640700\end{array}$ $\begin{array}{lll}\text { Au } 0.52880900 & 2.52790100 & 0.18726300\end{array}$ $\begin{array}{lll}\text { Au } 3.16716300 & -2.04883500 & 0.04136100\end{array}$ $\mathrm{H}=-875.672271$ Hartree $G=-875.747399$ Hartree

\section{Structure 4}

N -5.08707400 $-2.54794200 \quad-0.87709200$

C $-4.92498900-1.07362600-0.54238100$

H -5.31729700 $-0.48616700-1.37396700$

C -5.66255100 $-0.71329500 \quad 0.76430900$

$\begin{array}{lll}\text { C }-3.40216600 & -0.81732500 & -0.38315500\end{array}$

H -5.23437700 $-1.23420000 \quad 1.62472600$

$\begin{array}{lll}H-5.58792900 & 0.36182000 & 0.92606300\end{array}$

$\begin{array}{lll}S-7.49438400 & -1.21417400 & 0.76693800\end{array}$

$\begin{array}{llll}0 & -3.08186400 & 0.44790300 & -0.39572300\end{array}$

H $-7.92489500-0.32029900-0.18711900$

$\begin{array}{lll}\mathrm{O}-2.63789400 & -1.81999100 & -0.22847300\end{array}$

H $-4.28057600 \quad-3.04527000 \quad-0.45360200$

H -5.98591900 $-2.90598500-0.51816100$

H -5.05756700 $-2.70717100-1.89172700$

$\mathrm{Au}-1.04990400 \quad 1.14216800-0.16322600$ Au $1.48614800 \quad-0.16881000 \quad 0.03141500$ 
Au $1.16675900 \quad 2.53997500 \quad 0.08543000$

$\begin{array}{lll}\text { Au } 2.50591600 & -2.57406800 & 0.06794200\end{array}$ $\mathrm{H}=-875.671508$ Hartree

$G=-875.746410$ Hartree

Structure 5

N -5.64977500 $-0.53258800 \quad 0.20763900$

C - $5.05321000 \quad 0.62202200-0.58256600$

H -5.57519900 $0.68700100-1.53984500$

$\begin{array}{lll}C-5.19205300 & 1.95185400 & 0.19006500\end{array}$

C-3.55157500 $0.31714800 \quad-0.83135700$

H-4.61876900 $1.93912200 \quad 1.12057700$

H $-4.83151700 \quad 2.76585400-0.43884100$

S -6.97150800 $2.33241600 \quad 0.73419500$

O -2.92990800 $0.97139400 \quad-1.70791200$

H -7.49703500 $2.56003000-0.51737300$

O -3.07319000 $-0.61179000-0.02264600$

H $-4.88784200 \quad-0.92465200 \quad 0.79067300$

$\begin{array}{lll}H-6.44375800 & -0.20649700 & 0.78109600\end{array}$

H -5.98305200 $-1.27823300-0.41526700$

Au -1.00503300 - $-1.21757500-0.02604200$

Au $1.46125500 \quad 0.24047200 \quad 0.02912000$

$\begin{array}{lll}\text { Au } 1.29993400 & -2.48246200 & 0.08479600\end{array}$

Au $2.31644500 \quad 2.70822100 \quad 0.01600000$

$\mathrm{H}=-875.671290$ Hartree

$G=-875.745990$ Hartree

Structure 6

$\begin{array}{ccc}\text { N }-5.67948500 & 0.04116800 & -0.79818300 \\ \text { C-4.88298300 } & 1.32369400 & -0.97419900 \\ \text { H-4.89989600 } & 1.57290300 & -2.04142800 \\ \text { C-5.48497200 } & 2.49608000 & -0.18857800 \\ \text { C-3.40377600 } & 1.01776800 & -0.60149500 \\ \text { H-4.80761100 } & 3.34586800 & -0.26500700 \\ \text { H-6.46500400 } & 2.77481400 & -0.57801800 \\ \text { S-5.67569700 } & 2.09671500 & 1.66954800 \\ \text { O -3.13431600 } & -0.27605700 & -0.61280500 \\ \text { H-7.04986100 } & 2.12534600 & 1.72011900 \\ \text { O -2.61107500 } & 1.96554800 & -0.36766600 \\ \text { H -5.00812300 } & -0.73737900 & -0.94176200 \\ \text { H -6.02811500 } & -0.01461600 & 0.17292200 \\ \text { H -6.46963000 } & -0.02822400 & -1.44846300 \\ \text { Au -1.17019300 } & -1.07065700 & -0.21991600 \\ \text { Au } 1.42723900 & 0.10984400 & 0.02898200 \\ \text { Au } 0.94635000 & -2.56758900 & 0.23537200\end{array}$

Au $2.59267100 \quad 2.44719300 \quad-0.03576000$ $\mathrm{H}=-875.670257$ Hartree

$G=-875.745713$ Hartree

Structure 7

$\begin{array}{ccc}\text { N-5.00542600 } & -2.76318100 & -0.49300400 \\ \text { C-4.87616700 } & -1.24848300 & -0.49953800 \\ \text { H-5.19134200 } & -0.87705000 & -1.47640700 \\ \text { C-5.72220400 } & -0.61028200 & 0.62034500 \\ \text { C-3.37281400 } & -0.92837000 & -0.27517700 \\ \text { H-5.38038800 } & -0.92547400 & 1.60842700 \\ \text { H-5.64431600 } & 0.47334100 & 0.54051700 \\ \text { S-7.56405400 } & -1.09014300 & 0.45594100 \\ \text { O-3.06387600 } & 0.31881800 & -0.50162100 \\ \text { H-7.75302200 } & -1.50241800 & 1.75358300 \\ \text { O-2.61380800 } & -1.86539500 & 0.12498900 \\ \text { H-4.94462200 } & -3.15034800 & -1.44208600 \\ \text { H-4.20206600 } & -3.12940400 & 0.05461200 \\ \text { H-5.90867000 } & -3.05048600 & -0.08614400 \\ \text { Au -1.07040500 } & 1.09709800 & -0.20043000 \\ \text { Au 1.09861300 } & 2.55531100 & 0.11416500 \\ \text { Au 1.49977600 } & -0.14203500 & 0.03110200 \\ \text { Au 2.57753400 } & -2.52250300 & 0.04431200 \\ \text { H=-875.669548 Hartree } & \\ \text { G=-875.745444 Hartree } & \end{array}$

Structure 8

$\begin{array}{lcc}\text { N-5.50756200 } & -0.68099200 & 0.63585000 \\ \text { C-5.03796600 } & 0.42170700 & -0.30736700 \\ \text { H-5.44616500 } & 0.17956400 & -1.29433900 \\ \text { C-5.50794200 } & 1.81623600 & 0.12089800 \\ \text { C-3.48734200 } & 0.32095200 & -0.41664400 \\ \text { H-5.18457400 } & 2.06861700 & 1.13393300 \\ \text { H-5.07727600 } & 2.54353000 & -0.56767200 \\ \text { S-7.39330000 } & 2.04718900 & 0.00002800 \\ \text { O-2.83378400 } & 1.27336800 & -0.91361400 \\ \text { H-7.71099300 } & 1.75423100 & 1.30640600 \\ \text { O-3.02048300 } & -0.83736900 & 0.01729000 \\ \text { H-4.78793200 } & -1.42818800 & 0.56947200 \\ \text { H-5.53341400 } & -0.36403900 & 1.61295700 \\ \text { H-6.43571600 } & -1.03806500 & 0.38263000 \\ \text { Au -0.91864600 } & -1.32245800 & -0.01851000 \\ \text { Au } 1.46989000 & -2.42885000 & -0.00079900 \\ \text { Au 1.44786700 } & 0.29843300 & 0.01919100 \\ \text { Au 2.15348900 } & 2.81306400 & 0.04055400\end{array}$


$\mathrm{H}=-875.668587$ Hartree

$G=-875.744922$ Hartree

Structure 9

N $4.55813100 \quad-2.15120100 \quad 1.54452400$

C $5.23486400-1.02904500 \quad 0.77464500$

H $5.81345900-0.44229700 \quad 1.49717800$

C $6.19083600-1.55443100-0.30542900$

C $4.12049400 \quad-0.09622200 \quad 0.21910500$

H $6.54566800 \quad-0.70778600-0.89217600$

H $7.04302100 \quad-2.07439200 \quad 0.13481500$

S $5.32446000-2.75210900-1.51492400$

O $2.96217300 \quad-0.26340500 \quad 0.83285500$

H $6.02027300-3.88823000-1.17322300$

$\begin{array}{llll}0 & 4.39394100 & 0.72680500 & -0.69171000\end{array}$

H $3.64841000-1.76946400 \quad 1.86871500$

H $4.36356600 \quad-2.93848100 \quad 0.90412100$

H $5.12046700 \quad-2.48725500 \quad 2.33375900$

Au $1.19556300 \quad 0.85917500 \quad 0.31415500$

$\mathrm{Au}-1.49983800 \quad-0.04998700 \quad-0.03733700$

Au $-0.73530100 \quad 2.55896500 \quad-0.23924200$

$\mathrm{Au}-2.85636300 \quad-2.28244400 \quad 0.00666200$

$\mathrm{H}=-875.670212$ Hartree

$G=-875.744559$ Hartree

Structure 10

N -5.13523400 $-2.57358400 \quad-1.20694900$

C -5.00912900 $-1.09051000-0.90242300$

H -5.40991400 $-0.54173600 \quad-1.76185300$

$\begin{array}{lll}C-5.79803000 & -0.68176700 & 0.34952100\end{array}$

C -3.48396900 $-0.78927100-0.83655400$

H -5.57663300 $0.36122300 \quad 0.57097300$

H $-6.87160600-0.80343100 \quad 0.19834600$

S -5.29632400 $-1.70977800 \quad 1.87920100$

O -2.68660400 $-1.66462200-1.29929900$

H $-6.51561700 \quad-2.30362300 \quad 2.10602100$

O $-3.19139700 \quad 0.37664100-0.33204800$

H-4.23704700 $-2.85378600 \quad-1.65118200$

H $-5.23068300-3.10034400-0.32307700$

H -5.92968300 $-2.78851700-1.81861600$

$\mathrm{Au}-1.17023300 \quad 1.11763900-0.15846300$

Au $1.39881900 \quad-0.12733700 \quad 0.03645900$

$\begin{array}{lll}\text { Au } 1.00925500 & 2.57044500 & 0.10634900\end{array}$

Au $2.47392800 \quad-2.50916600 \quad 0.04664600$ $\mathrm{H}=-875.670244$ Hartree
$G=-875.744798$ Hartree

Structure 11

$\begin{array}{lll}\text { N } 5.49845300 & 0.24923300 & 1.26362900 \\ \text { C } 4.86529500 & 1.40112900 & 0.49921800 \\ \text { H } 4.96276700 & 2.30650600 & 1.10178000 \\ \text { C 5.54341200 } & 1.59453400 & -0.87290300 \\ \text { C } 3.35997200 & 1.08203600 & 0.29309600 \\ \text { H 5.41314900 } & 0.71842300 & -1.51155700 \\ \text { H 5.10269800 } & 2.46375200 & -1.36043500 \\ \text { S 7.41742000 } & 1.91529400 & -0.68708100 \\ \text { O 2.57691600 } & 2.00692100 & -0.04468300 \\ \text { H 7.85198200 } & 0.89966100 & -1.50527900 \\ \text { O 3.07713200 } & -0.19667400 & 0.46706600 \\ \text { H 5.41679400 } & 0.38372000 & 2.27842400 \\ \text { H 4.96866400 } & -0.60560700 & 1.01243900 \\ \text { H 6.49932600 } & 0.16466400 & 1.02752100 \\ \text { Au 1.10458600 } & -1.01296000 & 0.16515300 \\ \text { Au -1.00560700 } & -2.54947400 & -0.17831200 \\ \text { Au -1.51575900 } & 0.12540300 & -0.02457500 \\ \text { Au -2.70002700 } & 2.45388400 & 0.01506900 \\ \text { H= -875.669180 Hartree } & \\ \text { G= -875.744562 Hartree } & \end{array}$

Structure 12

$\begin{array}{ccc}\text { N } 3.08463500 & 4.12004800 & 1.08520200 \\ \text { H } 3.79886900 & 3.84539800 & 1.77013800 \\ \text { C } 2.37228700 & 2.90744800 & 0.51136100 \\ \text { H } 2.38252400 & 2.11274700 & 1.26018200 \\ \text { C } 0.92316600 & 3.25884500 & 0.11421500 \\ \text { C } 3.18552100 & 2.47776500 & -0.74785200 \\ \text { H } 0.89642300 & 4.00890900 & -0.67919300 \\ \text { H } 0.41396400 & 2.35493800 & -0.22289800 \\ \text { S-0.05518300 } & 3.94864400 & 1.60520800 \\ \text { O } 2.99312900 & 1.27650400 & -1.21895200 \\ \text { H-0.52850600 } & 5.08538000 & 0.99393600 \\ \text { H 2.41313600 } & 4.75071500 & 1.54979700 \\ \text { H 3.56516500 } & 4.58471800 & 0.28897700 \\ \text { O 3.96501700 } & 3.34682000 & -1.25199000 \\ \text { Au -3.18162400 } & 0.86593200 & -0.27656400 \\ \text { Au } 1.80621400 & -0.34996000 & -0.42430600 \\ \text { Au -1.01297700 } & -0.56516100 & -0.01580500 \\ \text { Au } 0.76557600 & -2.57881500 & 0.49212500 \\ \text { H= -875.667456 Hartree } & \\ \text { G=-875.744000 Hartree } & \end{array}$


Structure 13

$\begin{array}{ccc}\text { N } 6.34912900 & 1.04434300 & -1.18584000 \\ \text { C } 5.27149300 & 1.37513200 & -0.14857700 \\ \text { H } 5.72048200 & 1.21148200 & 0.83642900 \\ \text { C } 4.81103100 & 2.83931800 & -0.27852400 \\ \text { C } 4.15985100 & 0.30479600 & -0.35668300 \\ \text { H } 5.67679900 & 3.50125700 & -0.37030600 \\ \text { H } 4.16032500 & 2.97597600 & -1.14580300 \\ \text { S 3.91624700 } & 3.47254300 & 1.26484500 \\ \text { O } 3.03032900 & 0.56099300 & 0.24416700 \\ \text { H 2.91859100 } & 2.52376200 & 1.23314200 \\ \text { O } 4.44592200 & -0.71413500 & -1.06145300 \\ \text { H } 7.29687400 & 1.15033400 & -0.80757500 \\ \text { H 6.17077100 } & 0.05395000 & -1.46070300 \\ \text { H 6.26532700 } & 1.62939400 & -2.02653400 \\ \text { Au } 1.30873200 & -0.74478600 & 0.13493000 \\ \text { Au -1.45363200 } & -0.02970200 & -0.02569000 \\ \text { Au -0.55515200 } & -2.60240800 & 0.10177400 \\ \text { Au -2.97814100 } & 2.08819300 & -0.17246100 \\ \text { H= -875.668103 Hartree } & \\ \text { G= -875.743608 Hartree } & \end{array}$

Structure 14

$\begin{array}{lcc}\text { N } 5.38284400 & -0.27983800 & -1.18315200 \\ \text { C } 5.09750100 & 0.36800900 & 0.16849500 \\ \text { H } 5.63844000 & -0.22383200 & 0.91475500 \\ \text { C 5.54994700 } & 1.82994000 & 0.24121900 \\ \text { C } 3.57855900 & 0.19467300 & 0.46562800 \\ \text { H } 5.10865800 & 2.44077700 & -0.54974900 \\ \text { H } 5.23152800 & 2.23292200 & 1.20285100 \\ \text { S 7.44018300 } & 2.04083100 & 0.19804900 \\ \text { O 3.03901600 } & 0.87585900 & 1.37461900 \\ \text { H 7.58130700 } & 2.25447500 & -1.15343700 \\ \text { O 3.01399500 } & -0.73265800 & -0.28881900 \\ \text { H 4.66182200 } & -1.02028100 & -1.29323200 \\ \text { H 5.26786200 } & 0.38339800 & -1.96016300 \\ \text { H 6.32833700 } & -0.67638200 & -1.22967400 \\ \text { Au } 0.93654100 & -1.27548000 & -0.08070400 \\ \text { Au -1.46944000 } & 0.28351300 & -0.01698800 \\ \text { Au -1.41402800 } & -2.44196100 & 0.09597100 \\ \text { Au -2.23435100 } & 2.78093000 & -0.05848800 \\ \text { H= -875.668404 Hartree } & \\ \text { G=-875.742790 Hartree } & \end{array}$

Structure 15

$$
\begin{array}{lll}
\text { N }-5.10157100 & -2.55085300 & -1.12542600 \\
\text { C-4.95780800 } & -1.13519100 & -0.57277400 \\
\text { H -5.50616700 } & -0.47139400 & -1.24843100 \\
\text { C-5.49176000 } & -0.98082000 & 0.85619800 \\
\text { C-3.44338400 } & -0.78306900 & -0.69891900 \\
\text { H -5.04237800 } & -1.70002700 & 1.54578700 \\
\text { H-5.24122500 } & 0.02351000 & 1.19843100 \\
\text { S-7.38501400 } & -1.09081400 & 0.99410600 \\
\text { O -3.10020100 } & 0.31983100 & -0.09728600 \\
\text { H-7.49481200 } & -2.45313100 & 1.15312900 \\
\text { O -2.70846500 } & -1.56495900 & -1.38161600 \\
\text { H-4.23488700 } & -2.71071100 & -1.68517100 \\
\text { H -5.12962100 } & -3.25982900 & -0.38214600 \\
\text { H -5.93628400 } & -2.66098500 & -1.71130900 \\
\text { Au -1.08247300 } & 1.09119200 & -0.08854400 \\
\text { Au 1.10449400 } & 2.54938600 & 0.01794100 \\
\text { Au 1.48997300 } & -0.15017600 & 0.05025600 \\
\text { Au 2.56754500 } & -2.52968100 & 0.11434900 \\
\text { H= -875.668260 Hartree } & \\
\text { G=-875.742546 Hartree } &
\end{array}
$$

\section{Structure 16}

$$
\begin{array}{lll}
\text { N } 4.05724800 & -3.24146400 & 0.32463300 \\
\text { C } 4.95707800 & -2.00207600 & 0.36617900 \\
\text { H } 5.34984900 & -1.93941000 & 1.38641900 \\
\text { C } 6.11765300 & -2.12730000 & -0.63698900 \\
\text { C } 4.03604700 & -0.76963800 & 0.13066100 \\
\text { H } 6.57943700 & -3.11557000 & -0.56451500 \\
\text { H } 5.77635300 & -1.96476600 & -1.66212200 \\
\text { S 7.53492700 } & -0.92751600 & -0.26726500 \\
\text { O } 4.52906400 & 0.33380000 & -0.22589600 \\
\text { H 6.78058500 } & 0.21495800 & -0.41869200 \\
\text { O 2.76409200 } & -1.03171500 & 0.37025500 \\
\text { H 4.37053700 } & -3.96798900 & 0.97772600 \\
\text { H 3.10774500 } & -2.89871900 & 0.57953600 \\
\text { H 4.00790900 } & -3.64750100 & -0.61836800 \\
\text { Au 1.20334900 } & 0.44688900 & 0.16494500 \\
\text { Au -1.62684900 } & 0.05907500 & -0.02714200 \\
\text { Au -0.42061100 } & 2.50556800 & -0.05013100 \\
\text { Au -3.38300200 } & -1.87448200 & -0.06223000 \\
\text { H= -875.668108 Hartree } & \\
\text { G= -875.742733 Hartree } &
\end{array}
$$

Structure 17 


$\begin{array}{ccc}\text { N }-5.01662400 & 0.57906100 & 2.11066400 \\ \text { C-5.15108800 } & 0.73689700 & 0.59280900 \\ \text { H-5.87345700 } & -0.01900100 & 0.26664400 \\ \text { C-5.65966900 } & 2.14228400 & 0.22767300 \\ \text { C-3.76831700 } & 0.37090300 & -0.02084200 \\ \text { H-6.50764100 } & 2.41684900 & 0.86083400 \\ \text { H-4.87188700 } & 2.89149900 & 0.33650500 \\ \text { S-6.35035300 } & 2.23338500 & -1.53286800 \\ \text { O-3.46110500 } & 0.76113700 & -1.17830900 \\ \text { H-5.16793500 } & 1.89913900 & -2.15483400 \\ \text { O -3.03880700 } & -0.37860000 & 0.78677800 \\ \text { H-5.89255900 } & 0.27280100 & 2.54884300 \\ \text { H-4.26582300 } & -0.12775700 & 2.24935400 \\ \text { H-4.71611500 } & 1.45416600 & 2.55864200 \\ \text { Au -1.08399800 } & -1.11961000 & 0.25653100 \\ \text { Au } 1.07972900 & -2.51583400 & -0.28286300 \\ \text { Au } 1.45542100 & 0.17475600 & -0.02495200 \\ \text { Au 2.51709100 } & 2.56023100 & 0.06925500 \\ \text { H=-875.668024 Hartree } & \\ \text { G=-875.742382 Hartree } & \end{array}$

CYSTINE-Ag

Structure 1

$\begin{array}{lcc}\text { S } 3.57509400 & -0.66768800 & 1.72848000 \\ \text { S } 4.11248400 & -2.15524000 & 0.07758500 \\ \text { O }-0.49084300 & 2.15548100 & 0.02197000 \\ \text { O } 6.33834600 & 1.55431000 & 0.20355300 \\ \text { O }-0.87194100 & -0.11920900 & 0.20647200 \\ \text { O } 4.28215700 & 1.72803100 & -0.84525900 \\ \text { N } 2.07993100 & 2.02167500 & 0.55201200 \\ \text { N } 6.93159900 & -0.78092100 & -0.73594500 \\ \text { C } 1.40806900 & 0.68021500 & 0.34209200 \\ \text { C 5.70134700 } & -0.16051300 & -1.37657800 \\ \text { C } 1.71935000 & -0.32995800 & 1.45041600 \\ \text { C } 4.51404200 & -1.12582400 & -1.47879000 \\ \text { C }-0.12780700 & 0.93840400 & 0.18669100 \\ \text { C } 5.40838000 & 1.15922100 & -0.58544500 \\ \text { H } 1.76424900 & 0.30092600 & -0.62075400 \\ \text { H } 5.97469200 & 0.13757600 & -2.39501400 \\ \text { H } 1.37778700 & 0.02062600 & 2.43167100 \\ \text { H } 1.22667500 & -1.27604200 & 1.22901600 \\ \text { H } 3.63448000 & -0.55994900 & -1.78184800 \\ \text { H } 4.70327600 & -1.91280000 & -2.21737000\end{array}$

H $1.40075900 \quad 2.72511500 \quad 0.20132000$

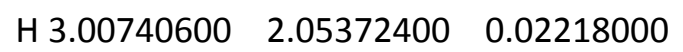

H $7.61769200-1.11579700-1.42001800$

H $6.66619300-1.56045100-0.11446500$

H $2.25067700 \quad 2.21756700 \quad 1.54464000$

H $7.32885400-0.00479200-0.15368000$

Ag -3.14617000 $-0.08999000-0.01672300$

Ag -5.76525000 -0.20920800 - 0.24411500

$\mathrm{H}=-957.814385$ Hartree

$G=-957.892010$ Hartree

\section{Structure 2}

\begin{tabular}{|c|c|c|}
\hline S -3.98470100 & 1.39803600 & 1.22096300 \\
\hline S -4.91658100 & 1.67240600 & -0.84905500 \\
\hline O 0.92899900 & -0.20673200 & 0.41382100 \\
\hline O -5.64390200 & -2.09551700 & 1.13717500 \\
\hline O 0.46667100 & 1.92341900 & -0.36256300 \\
\hline O -3.58509500 & -1.96401400 & 0.08775400 \\
\hline $\mathrm{N}-1.50505900$ & -0.76838200 & 1.15858400 \\
\hline N-6.96875300 & -0.83970800 & -0.69691200 \\
\hline C - 1.37927000 & 0.48877400 & 0.32277000 \\
\hline C -5.54501300 & -1.18451700 & -1.09967100 \\
\hline C - 2.12473800 & 1.68514000 & 0.92080700 \\
\hline C - 4.79157900 & -0.01242800 & -1.73917700 \\
\hline C 0.14116400 & 0.79160900 & 0.10873100 \\
\hline$C-4.85880700$ & -1.79237100 & 0.17019900 \\
\hline H -1.79005200 & 0.24821500 & -0.66286200 \\
\hline H -5.59987700 & -1.98856600 & -1.84200700 \\
\hline H -1.74615500 & 1.94730300 & 1.91603500 \\
\hline H - 2.01020800 & 2.54971300 & 0.26757700 \\
\hline H -3.74539800 & -0.29350400 & -1.84940400 \\
\hline$H-5.20016500$ & 0.23238300 & -2.72603400 \\
\hline H - 0.59860000 & -1.26146900 & 1.04269700 \\
\hline H -2.33070800 & -1.35673600 & 0.82245000 \\
\hline H -7.66370500 & -1.11623800 & -1.39787200 \\
\hline H -7.06029900 & 0.17081900 & -0.51020200 \\
\hline H -1.62328400 & -0.55719100 & 2.15571000 \\
\hline H -7.10858500 & -1.36145700 & 0.20109500 \\
\hline Ag 3.18800500 & -0.14571300 & 0.08018500 \\
\hline Ag 5.79561900 & -0.19892900 & -0.26977800 \\
\hline \multicolumn{3}{|c|}{$\mathrm{H}=-957.814179$ Hartree } \\
\hline \multicolumn{3}{|c|}{$G=-957.891946$ Hartree } \\
\hline
\end{tabular}

Structure 3

$\begin{array}{lll}\text { S }-3.95006000 & -2.12012800 & 0.92041700\end{array}$ 


\begin{tabular}{|c|c|c|}
\hline S - 2.26648700 & -0.81901600 & 0.08169300 \\
\hline 431200 & & \\
\hline O 0.13866400 & 1.70232300 & -0.92396500 \\
\hline O -4.63382100 & 1.59368400 & -0.09158900 \\
\hline O 0.96414200 & 0.89273300 & 1.08166300 \\
\hline N -6.13481400 & -1.45685400 & -1.40808400 \\
\hline N -2.16116700 & 2.50047100 & 0.05652900 \\
\hline C -5.98135700 & -0.40578400 & -0.32157200 \\
\hline C - 1.30804700 & 1.70295300 & 1.02600000 \\
\hline C -5.46666400 & -0.96840100 & 1.00754300 \\
\hline C - 1.99914800 & 0.42025400 & 1.52038400 \\
\hline C - 5.09470300 & 0.72973600 & -0.93200600 \\
\hline C 0.05674900 & 1.41321800 & 0.32239100 \\
\hline H -6.97203200 & 0.02567600 & -0.13971500 \\
\hline H -1.11195100 & 2.31940000 & 1.90812400 \\
\hline$H-5.24238300$ & -0.13147800 & 1.66885400 \\
\hline H -6.21168400 & -1.61185800 & 1.48847400 \\
\hline H -1.37043900 & -0.06155000 & 2.26893500 \\
\hline H -2.97987700 & 0.65158400 & 1.93985300 \\
\hline H -7.09034100 & -1.81962200 & -1.48447700 \\
\hline H -5.48710700 & -2.24364500 & -1.24823700 \\
\hline$H-2.19272000$ & 3.48825100 & 0.32988400 \\
\hline H -3.17424600 & 2.14591100 & -0.03922900 \\
\hline H -5.84925700 & -0.95832700 & -2.28434500 \\
\hline$H-1.66619500$ & 2.43323400 & -0.85666500 \\
\hline Ag 3.00457600 & 0.20166100 & 0.30378800 \\
\hline Ag 5.36662600 & -0.64790800 & -0.49008500 \\
\hline \multicolumn{3}{|c|}{$H=-957.812458$ Hartree } \\
\hline \multicolumn{3}{|c|}{$G=-957.890449$ Hartree } \\
\hline
\end{tabular}

Structure 4

$\begin{array}{lcc}\text { S } 4.70403700 & -2.16141300 & -0.35356700 \\ \text { S 3.58045600 } & -0.72130500 & -1.74209800 \\ \text { O } 3.81415500 & 1.36085900 & 1.18130700 \\ \text { O -0.46820100 } & 2.09248400 & -0.04140000 \\ \text { O } 5.72831500 & 1.88668000 & -0.01039800 \\ \text { O -0.93286000 } & 0.00876300 & -0.92955100 \\ \text { N } 4.80262700 & -0.61567600 & 2.48673900 \\ \text { N } 2.05779700 & 2.10609400 & -0.65426200 \\ \text { C 5.74640900 } & -0.06751400 & 1.42392700 \\ \text { C } 1.30882100 & 0.90144100 & -1.19338200 \\ \text { C } 6.12989300 & -1.11700600 & 0.36480400 \\ \text { C } 1.93889900 & -0.43744900 & -0.78654900 \\ \text { C } 5.05840000 & 1.18273000 & 0.79678200 \\ \text { C - }-16637300 & 1.01333900 & -0.66338400\end{array}$

$\begin{array}{lll}\text { H } 6.66521600 & 0.26047500 & 1.91792500 \\ \text { H } 1.25774700 & 0.98498000 & -2.28439600 \\ \text { H } 6.65148300 & -0.61966600 & -0.45379500 \\ \text { H } 6.78537600 & -1.88601800 & 0.78874200 \\ \text { H } 2.15580900 & -0.46999200 & 0.28151400 \\ \text { H } 1.25760700 & -1.24267700 & -1.05683300 \\ \text { H } 3.99033200 & 0.04867100 & 2.46170800 \\ \text { H } 5.22686700 & -0.63863300 & 3.41978700 \\ \text { H } 2.55165700 & 2.61702600 & -1.39155500 \\ \text { H } 2.76845500 & 1.85210800 & 0.11613700 \\ \text { H } 4.45877100 & -1.55608400 & 2.24644200 \\ \text { H } 1.31261300 & 2.70948500 & -0.24339300 \\ \text { Ag }-3.11805500 & -0.07853800 & -0.23931700 \\ \text { Ag }-5.62416500 & -0.30470900 & 0.52970300 \\ \text { H= -957.812756 Hartree } & \\ \text { G= -957.890184 Hartree } & \end{array}$

Structure 5

S $4.40990600 \quad-0.00246400-2.07333000$

S $2.33704800 \quad 0.20271800-1.12727100$

O $3.64149000 \quad-2.10290300 \quad 1.64386700$

$\begin{array}{llll}0-0.63294800 & 0.84794900 & 0.72810200\end{array}$

$\begin{array}{llll}0 & 4.02237100 & 0.17393200 & 1.81393800\end{array}$

$\begin{array}{llll}0 & -0.56121100 & 2.69049600 & -0.67284600\end{array}$

N $5.36269500-2.51751200-0.24613000$

N $1.64127200 \quad 1.31047300 \quad 1.90872200$

C $5.48335900-1.14920500 \quad 0.40322700$

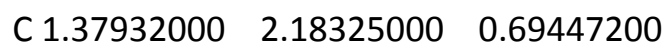

C $5.62705300-0.00493000-0.60554500$

C $2.42249500 \quad 1.98238300-0.41904300$

C $4.26069300-1.01464200 \quad 1.37066500$

C - $0.07398000 \quad 1.90436400 \quad 0.19417600$

H $6.38366700-1.16086600 \quad 1.02753300$

H $1.43641200 \quad 3.23221200 \quad 0.99944400$

H $5.54146300 \quad 0.93908700-0.06749900$

H $6.59571400-0.03778500-1.11663700$

H $2.22374300 \quad 2.68140100-1.23158800$

H $3.43114600 \quad 2.12998900-0.02920800$

H $6.25872800-3.00952100-0.32075800$

H $4.94368300-2.43745900-1.18554000$

H $1.55472500 \quad 1.85725100 \quad 2.77189900$

H $2.60480200 \quad 0.828431001 .90707900$

H $4.69774500 \quad-3.03585900 \quad 0.37496800$

H $0.88096000 \quad 0.600920001 .89868200$

Ag -2.73226000 $0.11779400 \quad 0.20394200$ 
Ag -5.13759300 $-0.83323400-0.27605000$ $\mathrm{H}=-957.812227$ Hartree

$\mathrm{G}=-957.889495$ Hartree

Structure 6

\begin{tabular}{|c|c|c|}
\hline S -3.74208300 & 0.40896200 & 1.58589800 \\
\hline S -1.63183900 & -0.49671700 & 1.72223100 \\
\hline O - 1.55897200 & 0.37268800 & -1.75530500 \\
\hline O 0.07446000 & -4.06868500 & -1.66836900 \\
\hline O -0.75962300 & 2.25321100 & -0.66569600 \\
\hline O -0.99839200 & -4.84760700 & 0.23379800 \\
\hline$N-4.07005300$ & 0.99365000 & -1.57629600 \\
\hline N 0.18991200 & -1.59438300 & -0.96054600 \\
\hline C -3.14569100 & 1.95526000 & -0.84152100 \\
\hline C -0.39598000 & -2.48800900 & 0.11287100 \\
\hline C -3.47105400 & 2.05525000 & 0.66116200 \\
\hline C - 1.78201700 & -2.04057200 & 0.58753700 \\
\hline C - 1.68434700 & 1.48928100 & -1.11025800 \\
\hline C -0.45720600 & -3.94896200 & -0.49309500 \\
\hline H -3.26928400 & 2.94946000 & -1.28034100 \\
\hline Н 0.30554200 & -2.52330400 & 0.95370000 \\
\hline H - 2.67873600 & 2.61535100 & 1.15843500 \\
\hline$H-4.42520100$ & 2.56935900 & 0.82092200 \\
\hline H - 2.43387900 & -1.79110800 & -0.25101100 \\
\hline H - 2.22449900 & -2.83925900 & 1.18132100 \\
\hline H -3.43120600 & 0.36452800 & -2.11300200 \\
\hline H -4.72199600 & 1.47425400 & -2.20491900 \\
\hline H 1.03783200 & -1.09021800 & -0.63605200 \\
\hline H -0.48211900 & -0.86495400 & -1.32028600 \\
\hline H -4.60488200 & 0.41275000 & -0.91358300 \\
\hline H 0.43801900 & -2.27224800 & -1.72198100 \\
\hline Ag 1.41723700 & 1.54928100 & -0.18881100 \\
\hline Ag 3.50174500 & 0.04208600 & 0.36964100 \\
\hline \multicolumn{3}{|c|}{$H=-957.813106$ Hartree } \\
\hline 0578807 & & \\
\hline
\end{tabular}

Structure 7

$\begin{array}{lcc}\text { S } 4.33336800 & -1.55991300 & -1.51030400 \\ \text { S } 3.49097000 & -2.71599600 & 0.27420000 \\ \text { O } 5.48840900 & 3.12916000 & 0.50179600 \\ \text { O }-0.31798900 & 0.26489000 & 2.08498600 \\ \text { O } 4.10945900 & 1.57841200 & 1.51010100 \\ \text { O }-0.72580500 & -0.48935900 & -0.06493700 \\ \text { N } 5.50848300 & 2.06791400 & -1.83203100 \\ \text { N } 2.24469600 & -0.23499500 & 2.06321200\end{array}$

\begin{tabular}{|c|c|c|}
\hline C 4.74866900 & 1.22970900 & -0.80854500 \\
\hline & -0.615 & 8200 \\
\hline C 5.34145100 & -0.18426800 & -0.63483700 \\
\hline C 1.69310100 & -2.09349400 & 0.40872100 \\
\hline C 4.78772400 & 2.05510600 & 0.52821800 \\
\hline C 0.02263900 & -0.24415400 & 0.95951300 \\
\hline H 3.70870900 & 1.17380600 & -1.13575100 \\
\hline H 1.93047700 & 0.00785100 & -0.02497100 \\
\hline H 6.35880300 & -0.26024500 & -1.02890300 \\
\hline H 5.36520800 & -0.45748900 & 0.42079700 \\
\hline H 1.26782100 & -2.75689200 & 1.17176700 \\
\hline H 1.19155900 & -2.28032600 & -0.54028800 \\
\hline H 6.27898800 & 1.56146000 & -2.28081300 \\
\hline H 5.88353300 & 2.86589900 & -1.25330700 \\
\hline H 2.99613600 & 0.49864200 & 1.87156000 \\
\hline H 2.68815200 & -1.04149000 & 2.51631100 \\
\hline H 4.89375100 & 2.43769800 & -2.56511800 \\
\hline H 1.49760400 & 0.14981500 & 2.68000000 \\
\hline $\mathrm{Ag}-2.97206000$ & $\begin{array}{ll}0 & -0.05247300\end{array}$ & $0 \quad-0.12628900$ \\
\hline $\mathrm{Ag}-5.55376600$ & $0 \quad 0.41275300$ & $\begin{array}{ll}0 & -0.32768000\end{array}$ \\
\hline \multicolumn{3}{|c|}{$H=-957.810759$ Hartree } \\
\hline & & \\
\hline
\end{tabular}

Structure 8

\begin{tabular}{|c|c|c|}
\hline$S-3.45322600$ & 0.89434800 & 1.64818600 \\
\hline S -1.80414500 & -0.72727900 & 1.78554000 \\
\hline O -0.84299500 & 0.44493200 & -1.46385700 \\
\hline O -3.95817300 & -0.46316800 & -1.63642800 \\
\hline O 0.29332300 & 1.84813300 & -0.03619700 \\
\hline O -5.32992800 & -1.76595500 & -0.31459200 \\
\hline N -3.07489700 & 1.98110900 & -1.48835500 \\
\hline N -1.79825200 & -2.02878800 & -1.28607800 \\
\hline C - 2.00204700 & 2.35936000 & -0.49106300 \\
\hline C - 2.99682900 & -2.33269800 & -0.40880800 \\
\hline C -2.50856800 & 2.39595400 & 0.96249300 \\
\hline C - 2.67321600 & -2.27318600 & 1.09333300 \\
\hline C - 0.75090700 & 1.46201700 & -0.68143800 \\
\hline C -4.20735800 & -1.43987100 & -0.81300100 \\
\hline H -1.68839000 & 3.38303800 & -0.72717200 \\
\hline H -3.28717600 & -3.36944600 & -0.61499000 \\
\hline H -1.65749000 & 2.59981800 & 1.61407400 \\
\hline H -3.24546600 & 3.19643800 & 1.09794200 \\
\hline H -3.60074700 & -2.42674300 & 1.64747800 \\
\hline H -1.96702600 & -3.06282000 & 1.37665000 \\
\hline H - 2.71831600 & 2.12543000 & -2.44064800 \\
\hline
\end{tabular}




\author{
H -3.90624200 $2.57129100 \quad-1.36827700$ \\ H $-0.98932800-2.60301700-1.02053200$ \\ $\mathrm{H}-1.50736300-1.00240200-1.27026000$ \\ H $-3.38279800 \quad 0.94269100-1.44087400$ \\ H -2.03403900 $-2.24054400 \quad-2.26276700$ \\ Ag $2.24781700 \quad 0.62553700 \quad-0.03674500$ \\ Ag $4.51282300 \quad-0.71051700 \quad 0.02675000$ \\ $\mathrm{H}=-957.812487$ Hartree \\ $\mathrm{G}=-957.888446$ Hartree
}

Structure 9

\begin{tabular}{|c|c|c|}
\hline 3.99810800 & -2.37673800 & 0.69822200 \\
\hline 4.98020600 & -1.15075700 & -0.95569600 \\
\hline-0.56664500 & -2.06214100 & 1.21419400 \\
\hline O 4.06739300 & 2.99729400 & 1.22152500 \\
\hline O - 0.82697600 & -0.58797200 & -0.54918900 \\
\hline O 2.87946600 & 1.41231900 & 0.05466600 \\
\hline N 1.65659200 & -0.53154300 & -1.25739000 \\
\hline N 6.27306500 & 2.75244600 & -0.00925000 \\
\hline C 1.34176400 & -1.60901600 & -0.23457900 \\
\hline C 5.26505400 & 1.66852900 & -0.40324800 \\
\hline C 2.29059000 & -1.57731900 & 0.96714900 \\
\hline C 5.83381600 & 0.27876500 & -0.02863400 \\
\hline C - 0.14934700 & -1.40948800 & 0.21054300 \\
\hline C 3.94943000 & 2.05446400 & 0.35372000 \\
\hline 9500 & -2.58191300 & -0.73433700 \\
\hline H 5.10387100 & 1.73083500 & -1.48158300 \\
\hline H 2.43581400 & -0.56289200 & 1.34083900 \\
\hline H 1.86506900 & -2.20216700 & 1.75915100 \\
\hline H 6.88208300 & 0.20973800 & -0.33490600 \\
\hline H 5.76089500 & 0.07555200 & 1.04221100 \\
\hline H 2.18946600 & -0.89269600 & -2.05327800 \\
\hline H 2.20381500 & 0.26961800 & -0.81562200 \\
\hline H 6.47849300 & 3.38815900 & -0.78752900 \\
\hline H 7.15893800 & 2.37466600 & 0.34415600 \\
\hline H 0.72571800 & -0.18189800 & -1.56470800 \\
\hline H 5.76365200 & 3.27571500 & 0.75377500 \\
\hline $\mathrm{Ag}-3.02279900$ & $0-0.07345200$ & $0-0.19648700$ \\
\hline $\mathrm{Ag}-5.54606200$ & 0.61556400 & 0.08581900 \\
\hline \multicolumn{3}{|c|}{$H=-957.810261$ Hartree } \\
\hline \multicolumn{3}{|c|}{$G=-957.888270$ Hartree } \\
\hline
\end{tabular}

Structure 10

S $5.47834000 \quad 1.04033700-1.24477900$ S $4.08409900 \quad 1.80342600 \quad 0.40891600$

\begin{tabular}{|c|c|c|}
\hline 3.11988400 & -1.66814900 & 0.39 \\
\hline & & \\
\hline O 4.82981900 & -1.58113800 & 1.95525000 \\
\hline O -0.45601200 & 2.13867700 & -0.59815200 \\
\hline N 4.60466200 & -2.04481900 & -1.66312000 \\
\hline N 1.50142100 & 0.00947500 & 1.65808000 \\
\hline C 5.39987500 & -1.77049300 & -0.39365400 \\
\hline C 1.32304000 & 1.26579300 & 0.82561500 \\
\hline C 6.29137600 & -0.52038100 & -0.50727600 \\
\hline C 2.35153500 & 1.39000300 & -0.30657200 \\
\hline C 4.37792900 & -1.66726200 & 0.77856300 \\
\hline C - 0.13772900 & 1.24975900 & 0.24649900 \\
\hline H 6.05021700 & -2.62910800 & -0.20416400 \\
\hline H 1.38144400 & 2.13197200 & 1.49398700 \\
\hline H 6.69212800 & -0.27975200 & 0.47802600 \\
\hline H 7.12371600 & -0.69185900 & -1.19883800 \\
\hline H 2.43128500 & 0.46256600 & -0.87446200 \\
\hline H 2.05867000 & 2.21017500 & -0.96049000 \\
\hline H 3.61260300 & -2.10797000 & -1.32761500 \\
\hline H 4.88917500 & -2.91117800 & -2.13164000 \\
\hline H 1.90066200 & 0.20824300 & 2.58000000 \\
\hline H 2.12196600 & -0.72608000 & 1.17298900 \\
\hline H 4.66926700 & -1.26230300 & -2.32946700 \\
\hline H 0.53643700 & -0.37038700 & 1.76028500 \\
\hline Ag -3.08897200 & $0 \quad 0.00574600$ & $0 \quad 0.18575700$ \\
\hline Ag -5.62709800 & $0-0.39945400$ & $0-0.37423400$ \\
\hline \multicolumn{3}{|c|}{$H=-957.812354$ Hartree } \\
\hline \multicolumn{3}{|c|}{$=-957.888450$ Hartree } \\
\hline
\end{tabular}

Structure 11

$\begin{array}{lcc}\text { S } 3.23077800 & -2.21884700 & 1.10064600 \\ \text { S } 4.94823500 & -1.63580900 & -0.27980800 \\ \text { O }-1.00025100 & -0.92203900 & -0.02622300 \\ \text { O } 4.71965700 & 3.08173300 & 0.64901000 \\ \text { O }-0.36302500 & 0.23644200 & -1.92372900 \\ \text { O } 3.47604400 & 1.47896600 & -0.43164400 \\ \text { N } 2.16307800 & -0.37897800 & -1.77691800 \\ \text { N } 6.99131800 & 1.98015300 & 0.43545100 \\ \text { C } 1.29264700 & -1.13035700 & -0.78513600 \\ \text { C } 5.86575900 & 1.09059700 & -0.10256800 \\ \text { C } 1.81244300 & -1.02984300 & 0.65174000 \\ \text { C 5.84371200 } & -0.24762700 & 0.67300600 \\ \text { C - }-1.15431500 & -0.53895900 & -0.92494700 \\ \text { C } 4.56697800 & 1.95393800 & 0.04767500 \\ \text { H } 1.23979400 & -2.17862200 & -1.09701900\end{array}$




$\begin{array}{ccc}\text { H } 6.06015100 & 0.90753100 & -1.16189400 \\ \text { H } 2.11783600 & -0.01285700 & 0.90263000 \\ \text { H } 1.01664800 & -1.35261300 & 1.33020300 \\ \text { H } 6.86362800 & -0.62086100 & 0.80400300 \\ \text { H } 5.36850100 & -0.15234200 & 1.65187400 \\ \text { H } 2.77022800 & -1.00341600 & -2.31424700 \\ \text { H } 2.76935400 & 0.34958200 & -1.28823300 \\ \text { H } 7.65775300 & 2.24917200 & -0.29624800 \\ \text { H } 7.51271200 & 1.55120000 & 1.20783400 \\ \text { H } 1.48701500 & 0.10430900 & -2.40527500 \\ \text { H } 6.46540100 & 2.83022100 & 0.78041300 \\ \text { Ag -3.17472600 } & -0.20910000 & 0.04312100 \\ \text { Ag -5.68265200 } & 0.55394500 & 0.26847200 \\ \text { H= -957.810296 Hartree } & \\ \text { G= -957.887918 Hartree } & \end{array}$

Structure 12

\begin{tabular}{|c|c|c|}
\hline S - 1.82761800 & -0.7 & 1. \\
\hline S -3.47155300 & 0.91143600 & 1.63061400 \\
\hline-3.94309000 & -0.47598100 & -1.64977900 \\
\hline O - 0.83224000 & 0.42900400 & -1.45070300 \\
\hline O -5.33202900 & -1.76301700 & -0.33033800 \\
\hline O 0.29549600 & 1.84564700 & -0.02995900 \\
\hline N -1.79072100 & -2.04329700 & -1.26147900 \\
\hline N -3.06173200 & 1.96980400 & -1.50846900 \\
\hline C-2.99946900 & -2.33620400 & -0.39 \\
\hline C - 1.99607100 & 2.35477500 & -0.50599100 \\
\hline C - 2.69203200 & -2.26324200 & 1.11042800 \\
\hline C - 2.51473200 & 2.40434400 & 0.94288800 \\
\hline C -4.20341800 & -1.44430500 & -0.81994800 \\
\hline C -0.74486100 & 1.45408300 & -0.67838800 \\
\hline H -3.29005200 & -3.37414000 & -0.59422100 \\
\hline H -1.67890900 & 3.37594300 & -0.74819400 \\
\hline H -3.62581200 & -2.40987700 & 1.65586100 \\
\hline H -1.99045900 & -3.05146600 & 1.40901700 \\
\hline$H-1.66851300$ & 2.60941300 & 1.60041200 \\
\hline H -3.24924600 & 3.20894800 & 1.06616800 \\
\hline H -2.01740100 & -2.26119300 & -2.23897900 \\
\hline H - 0.98676700 & -2.61859900 & -0.98348000 \\
\hline H -3.89516200 & 2.55857900 & -1.39596800 \\
\hline H -3.36800100 & 0.93109700 & -1.45810900 \\
\hline H -1.49627200 & -1.01800500 & -1.25013100 \\
\hline H -2.69980700 & 2.11085400 & -2.45920700 \\
\hline Ag 2.25087600 & 0.62402600 & -0.03092500 \\
\hline Ag 4.51760700 & -0.70986200 & 0.02455000 \\
\hline
\end{tabular}

\author{
$\mathrm{H}=-957.812515$ Hartree \\ $G=-957.887635$ Hartree
}

Structure 13

\begin{tabular}{|c|c|c|}
\hline S 2.01766700 & 1.11901700 & 0.10660100 \\
\hline S 3.93130700 & 2.19408100 & 0.72720400 \\
\hline-1.10339200 & -0.88242500 & 1.06458500 \\
\hline O 6.22153300 & -1.65288900 & -1.19305900 \\
\hline O - 0.20147200 & -1.45332900 & -0.98926800 \\
\hline O 4.50987400 & -1.19100800 & 0.28658500 \\
\hline N 2.12356200 & -2.22219300 & -0.01728200 \\
\hline N 7.45901800 & 0.55936800 & -0.94163000 \\
\hline C 1.22516900 & -1.50757100 & 0.97264600 \\
\hline C 6.16016000 & 0.52148900 & -0.12801200 \\
\hline C 1.85703300 & -0.20365300 & 1.48956000 \\
\hline C 5.21840900 & 1.66417400 & -0.56977200 \\
\hline C - 0.15119000 & -1.26966700 & 0.27840600 \\
\hline C 5.57744200 & -0.90782100 & -0.36689100 \\
\hline H 1.07003700 & -2.15804600 & 1.83837400 \\
\hline H 6.42079600 & 0.63646900 & 0.92605900 \\
\hline H 1.22051600 & 0.21588600 & 2.26877400 \\
\hline H 2.86538600 & -0.37867900 & 1.86521900 \\
\hline H 5.79259200 & 2.58669200 & -0.71202900 \\
\hline H 4.69115900 & 1.43307900 & -1.49822600 \\
\hline H 1.70720700 & -2.04704700 & -0.95180400 \\
\hline H 2.11139500 & -3.23423400 & 0.14634500 \\
\hline H 8.28531900 & 0.68467600 & -0.34720300 \\
\hline H 7.46425400 & 1.28942500 & -1.66287900 \\
\hline H 3.13669500 & -1.87553200 & 0.03359800 \\
\hline Н 7.47676800 & -0.39169300 & -1.39881200 \\
\hline Ag -3.16221900 & $\begin{array}{ll}0 & -0.24853300\end{array}$ & $0 \quad 0.28632800$ \\
\hline $\mathrm{Ag}-5.54497800$ & $0 \quad 0.53629300$ & $0-0.51063100$ \\
\hline \multicolumn{3}{|c|}{$H=-957.808538$ Hartree } \\
\hline \multicolumn{3}{|c|}{$G=-957.887471$ Hartree } \\
\hline
\end{tabular}

Structure 14

$\begin{array}{lcc}\text { S } 2.64161600 & -1.00092100 & -2.05322200 \\ \text { S } 0.95694000 & 0.31810800 & -1.07147200 \\ \text { O } 1.74043300 & -1.56408300 & 2.14327400 \\ \text { O }-1.05611400 & 2.74693600 & 0.63129600 \\ \text { O } 3.04625500 & 0.27789200 & 1.63607200 \\ \text { O - } 0.38528300 & 4.13510800 & -1.09429200 \\ \text { N } 2.79436600 & -3.12995900 & 0.36992100 \\ \text { N } 1.39775700 & 2.33316600 & 1.53652200 \\ \text { C 3.57297500 } & -1.84433200 & 0.59090400\end{array}$




$\begin{array}{ccc}\text { C } 1.31441900 & 2.93434600 & 0.14507400 \\ \text { C } 3.98820100 & -1.15626000 & -0.71530800 \\ \text { C } 1.88414100 & 2.00216700 & -0.94210600 \\ \text { C } 2.69708900 & -0.96086400 & 1.53887100 \\ \text { C }-0.16901400 & 3.32643900 & -0.14177600 \\ \text { H } 4.48379000 & -2.10122700 & 1.14310600 \\ \text { H } 1.92044400 & 3.84470200 & 0.12516300 \\ \text { H } 4.37268000 & -0.16573100 & -0.47147300 \\ \text { H } 4.76947400 & -1.72289300 & -1.23420000 \\ \text { H } 1.81258200 & 2.50079300 & -1.90946300 \\ \text { H } 2.92616600 & 1.76362100 & -0.72098300 \\ \text { H } 3.38053400 & -3.97004500 & 0.40508600 \\ \text { H 2.30258400 } & -3.09629800 & -0.53743400 \\ \text { H } 1.69267300 & 3.04241200 & 2.21629200 \\ \text { H 2.06525800 } & 1.48950300 & 1.61520000 \\ \text { H } 2.08454900 & -3.13419400 & 1.13939700 \\ \text { H } 0.43003200 & 2.04639200 & 1.77650600 \\ \text { Ag -1.91275100 } & 0.62784100 & 0.23133200 \\ \text { Ag }-2.88834900 & -1.78891000 & -0.15222400 \\ \text { H= -957.810654 Hartree } & \\ \text { G=-957.887251 Hartree } & \end{array}$

Structure 15

$\begin{array}{lll}\text { S } 3.36250600 & -2.07134000 & -0.63951100 \\ \text { S } 4.62688100 & -1.72586400 & 1.23919000 \\ \text { O }-1.06874800 & -0.63384000 & -0.64009700 \\ \text { O } 5.53940100 & 2.27664300 & 1.53968500 \\ \text { O }-0.40887500 & 1.50683000 & -1.21733400 \\ \text { O } 3.64859200 & 1.53930900 & 0.44304100 \\ \text { N } 2.09910600 & 0.92268300 & -1.61243300 \\ \text { N } 7.14132100 & 1.31462700 & -0.31176300 \\ \text { C } 1.23002500 & -0.28375200 & -1.32024700 \\ \text { C } 5.81201800 & 0.57523600 & -0.13680400 \\ \text { C } 1.76538700 & -1.12077300 & -0.15350600 \\ \text { C } 6.11676900 & -0.75189200 & 0.58873400 \\ \text { C -0.21407500 } & 0.25457600 & -1.02613900 \\ \text { C } 4.91433800 & 1.55001400 & 0.68797000 \\ \text { H } 1.16118200 & -0.89213000 & -2.22808400 \\ \text { H } 5.38870900 & 0.38615400 & -1.12393500 \\ \text { H } 2.01312200 & -0.50098000 & 0.71077200 \\ \text { H } 1.02219600 & -1.86796100 & 0.12128300 \\ \text { H } 6.68588500 & -1.41621400 & -0.06606200 \\ \text { H } 6.68985300 & -0.57281000 & 1.50674500 \\ \text { H } 2.77846000 & 1.13709500 & -0.80762700 \\ \text { H } 1.42649200 & 1.71403700 & -1.70281300\end{array}$

H $7.94554700 \quad 0.68713900-0.42942800$

$\begin{array}{llll}\text { H } 7.23997400 & 1.88584500 & 0.55454900\end{array}$

H $2.63036700 \quad 0.81563600-2.48089500$

H $7.11048700 \quad 1.95204600-1.11721000$

Ag -3.24984700 $-0.16031500-0.12910500$

Ag $-5.77217800 \quad 0.22232000 \quad 0.51410100$

$\mathrm{H}=-957.807154$ Hartree

$\mathrm{G}=-957.887249$ Hartree

Structure 16

S - $1.97122500 \quad-0.07250900 \quad 1.50223700$

S -4.08533000 $-0.60701900 \quad 2.16391100$

$\begin{array}{llll}0 & 0.72686200 & 0.88313800 & -0.53489700\end{array}$

$\begin{array}{llll}0 & -4.13775100 & 0.69799200 & -1.06106200\end{array}$

$\begin{array}{llll}0 & 0.83817200 & 2.59083000 & 1.02455900\end{array}$

$\begin{array}{llll}0 & -5.55184700 & -0.35455800 & -2.55222500\end{array}$

N-1.66425500 $1.49251200-1.40804000$

N -6.76224300 $-1.81944100-0.85313900$

$\begin{array}{llll}C-1.26051400 & 2.18970200 & -0.12363800\end{array}$

C - $5.76550800-0.86077100-0.19405400$

C - $2.16335700 \quad 1.78440100 \quad 1.05437900$

$\begin{array}{lll}\text { C }-4.78947800 & -1.63103400 & 0.72050200\end{array}$

C $0.242224001 .88377600 \quad 0.15580900$

$\begin{array}{llll}\text { C }-5.08492500 & -0.10441700 & -1.38093900\end{array}$

H - $1.37273000 \quad 3.26905400-0.26255900$

$\begin{array}{lll}H-6.34463400 & -0.14369800 & 0.39333200\end{array}$

H - $1.88469500 \quad 2.36494800 \quad 1.93438900$

H-3.21549100 $1.93296000 \quad 0.81026900$

$\begin{array}{lll}H-5.31670300 & -2.44015900 & 1.23729500\end{array}$

H -3.94817900 $-2.05443200 \quad 0.16788000$

H $-0.99933200 \quad 0.70554900-1.52176600$

$H-1.54637700 \quad 2.11814300 \quad-2.21197700$

$\begin{array}{lll}H-6.73692600 & -1.52431200 & -1.86512800\end{array}$

H $-7.70804600 \quad-1.73327300-0.46736000$

H - $2.67517200 \quad 1.15054200 \quad-1.37710200$

H -6.47031600 $-2.80137500-0.78613700$

$\begin{array}{lll}\text { Ag } 2.85031100 & 0.09433100 & -0.26820700\end{array}$

Ag $5.27809000-0.89841900-0.05699800$

$\mathrm{H}=-957.808147$ Hartree

$G=-957.887143$ Hartree

Structure 17

S $1.99942600-1.14424000-0.03795700$

S $3.91700400-2.21468900-0.64240800$

O $-0.21105200 \quad 1.41399900 \quad 1.08100400$ 


\begin{tabular}{lcc} 
O 4.51935800 & 1.17614200 & -0.21472600 \\
O -1.10657400 & 0.89945900 & -0.99085400 \\
O 6.50741100 & 1.73483100 & 0.81751300 \\
N 2.12711500 & 2.18343100 & 0.13613600 \\
N 7.52654400 & -0.60216100 & 0.81556800 \\
C 1.22692600 & 1.49615000 & -0.87178200 \\
C 6.18544700 & -0.54759200 & 0.07577400 \\
C 1.84888400 & 0.19456100 & -1.40601900 \\
C 5.21546200 & -1.61846600 & 0.61779500 \\
C -0.15560200 & 1.25898400 & -0.19020000 \\
C 5.68914200 & 0.92536100 & 0.24527100 \\
H 1.08422700 & 2.16361400 & -1.72651700 \\
H 6.39300100 & -0.72864900 & -0.98211400 \\
H 1.20893900 & -0.20985200 & -2.19044100 \\
H 2.85835600 & 0.36684900 & -1.77990700 \\
H 5.76048900 & -2.54000800 & 0.84951800 \\
H 4.68740600 & -1.28543700 & 1.51370100 \\
H 1.71756400 & 1.97639100 & 1.06677500 \\
H 2.10715100 & 3.20028700 & 0.00595000 \\
H 7.70932000 & 0.41097000 & 1.04329400 \\
H 8.27940400 & -0.99226200 & 0.23928800 \\
H 3.13801600 & 1.84673600 & 0.06622000 \\
H 7.47411000 & -1.13463600 & 1.69164200 \\
Ag -3.18094000 & 0.26151200 & -0.26189600 \\
Ag -5.59334000 & -0.52639500 & 0.43334200 \\
H= -957.808364 Hartree & \\
G= -957.886946 Hartree & \\
\hline & &
\end{tabular}

Structure 18

\begin{tabular}{|c|c|c|}
\hline 00 & -1.09354500 & 400 \\
\hline-3.91765000 & -2.20171400 & 0.72257900 \\
\hline O 1.10022000 & 0.89759000 & 1.06495500 \\
\hline O -6.08179000 & 1.55927000 & -1.34614400 \\
\hline 0.19035600 & 1.47203500 & -0.98460100 \\
\hline O -4.52235500 & 1.22684700 & 0.32578000 \\
\hline N -2.13083900 & 2.24230300 & 0.00246900 \\
\hline N -7.46135300 & -0.54189500 & -0.87745400 \\
\hline C - 1.22990900 & 1.51676200 & 0.98193600 \\
\hline C - 6.14567200 & -0.50990600 & -0.09282400 \\
\hline C - 1.86101700 & 0.20644600 & 1.48341000 \\
\hline C -5.23505700 & -1.67559800 & -0.54379400 \\
\hline C 0.14488300 & 1.28519800 & 0.28274200 \\
\hline C -5.53163000 & 0.89244700 & -0.39564000 \\
\hline H -1.07358700 & 2.15676200 & 1.85516000 \\
\hline H -6.37645200 & -0.59984000 & 0.96975600 \\
\hline
\end{tabular}

$\begin{array}{lll}H-1.22170500 & -0.22576800 & 2.25331600\end{array}$

H $-2.86773500 \quad 0.37788000 \quad 1.86518300$

$\mathrm{H}-5.82462000-2.59291700-0.65262700$

H $-4.73477200 \quad-1.46969400 \quad-1.49292400$

$\mathrm{H}-1.72294700 \quad 2.07051900-0.93615200$

$\begin{array}{lll}H-2.11134100 & 3.25328300 & 0.17190100\end{array}$

H -8.27393000 $-0.42902800-0.26087100$

H -7.58992500 $-1.39782600-1.42786800$

H -3.14743300 $1.90068800 \quad 0.05888200$

H -7.38540000 $0.29786700-1.50819600$

$\begin{array}{lll}\text { Ag } 3.15453000 & 0.25251300 & 0.28610900\end{array}$

Ag $5.53238800-0.55163400-0.50684700$ $\mathrm{H}=-957.808370$ Hartree

$G=-957.886733$ Hartree

Structure 19

\begin{tabular}{|c|c|c|}
\hline S -4.86278600 & -1.58807800 & -0.83148100 \\
\hline S -3.20055400 & -2.29366100 & 0.60672100 \\
\hline O -6.15465300 & 2.73463100 & -0.03745100 \\
\hline O 0.41477800 & 0.95397300 & -1.55778500 \\
\hline O -4.24287200 & 1.79966000 & -0.91036400 \\
\hline O 0.96185900 & -0.49111200 & 0.16300600 \\
\hline N -6.31560500 & 1.22282400 & 2.01618600 \\
\hline$N-2.13276600$ & 0.39343400 & -1.57955400 \\
\hline C -5.36291000 & 0.67972200 & 0.94819600 \\
\hline C - 1.37831100 & -0.23025100 & -0.42375500 \\
\hline C -5.96304700 & -0.62019100 & 0.37029100 \\
\hline C -1.61200000 & -1.73624500 & -0.28450700 \\
\hline C -5.23418900 & 1.83813000 & -0.09516100 \\
\hline C 0.14175600 & 0.10426200 & -0.63875900 \\
\hline H -4.40193300 & 0.47246800 & 1.42198100 \\
\hline$H-1.68357000$ & 0.28628900 & 0.49195600 \\
\hline H -6.21603800 & -1.30856900 & 1.18125300 \\
\hline H -6.86746200 & -0.42149500 & -0.21674200 \\
\hline$H-1.55853600$ & -2.25086100 & -1.24818800 \\
\hline H -0.83302100 & -2.14355900 & 0.36777800 \\
\hline H -6.78699900 & 2.02721900 & 1.52790100 \\
\hline H -5.80448000 & 1.58063800 & 2.83130400 \\
\hline $\mathrm{H}-1.44191200$ & 1.03127500 & -2.02901000 \\
\hline H -3.00494200 & 0.93364400 & -1.26439800 \\
\hline$H-7.00350500$ & 0.53530300 & 2.34284700 \\
\hline$H-2.42053300$ & -0.30826000 & -2.26930900 \\
\hline $\mathrm{Ag} 3.21892900$ & -0.09141600 & 0.14569300 \\
\hline Ag 5.81924800 & 0.30780200 & 0.21356800 \\
\hline \multicolumn{3}{|c|}{$H=-957.809240$ Hartree } \\
\hline
\end{tabular}


$G=-957.886693$ Hartree

Structure 20

S $4.83720700-1.14675000 \quad 1.28915600$

S $3.88997200-2.39717000-0.40522700$

$\begin{array}{llll}0 & 5.25308700 & 3.27013800 & 0.11929700\end{array}$

O $-0.85523900-0.06619900 \quad 0.17048700$

$\begin{array}{llll}0 & 3.45781600 & 1.92216700 & 0.61847200\end{array}$

O - $0.57866900-1.86508600-1.25729100$

N $6.397354001 .66457500-1.50156900$

N $1.61709400 \quad 0.08438300 \quad 0.93247900$

C $5.31870100 \quad 0.98491600-0.65430500$

C $1.39415800-0.85076800-0.23801300$

C $5.99727600-0.04036800 \quad 0.27924000$

C $2.02680300-2.23148200-0.05037400$

$\begin{array}{lll}C 4.60669200 & 2.15806400 & 0.09593400\end{array}$

C - $0.15543800-0.96580200-0.47000300$

H $4.62377900 \quad 0.47734100-1.32599600$

H $1.80546600-0.36601000-1.12986100$

H $6.63559900-0.71155100-0.30216700$

H $6.60867000 \quad 0.45107300 \quad 1.04489400$

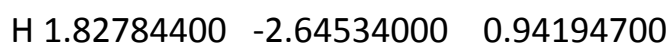

H $1.59237500-2.90626600-0.79540100$

H $6.471637002 .62109300-1.06701100$

H $6.10412600 \quad 1.76025100-2.48054700$

H $0.70697900 \quad 0.57646200 \quad 1.04864100$

H $2.41160500 \quad 0.78671700 \quad 0.76478800$

H $7.302662001 .18216900-1.48101900$

H $1.81667300 \quad-0.42876200 \quad 1.79747400$

$\begin{array}{lll}\text { Ag }-3.12962200 & 0.09594200 & 0.04975700\end{array}$

Ag $-5.74247400 \quad 0.39868200 \quad-0.01055600$

$\mathrm{H}=-957.809197$ Hartree

$G=-957.886748$ Hartree

Structure 21

$\begin{array}{lrc}\text { S }-4.32269300 & 0.20124900 & -0.85348600 \\ \text { S }-2.41284800 & -1.05288500 & -0.97375000 \\ \text { O }-0.56697600 & 2.16373800 & -0.00373600 \\ \text { O } 0.60611300 & -3.01933200 & -0.54598900 \\ \text { O }-1.39455200 & 0.80510600 & 1.67300400 \\ \text { O }-1.10490800 & -4.58688200 & -0.52836400 \\ \text { N }-2.86461200 & 3.10306300 & -0.75423300 \\ \text { N }-0.25780300 & -1.64600300 & 1.48009300 \\ \text { C }-2.90380200 & 2.34227300 & 0.56076700 \\ \text { C }-1.22365700 & -2.72258700 & 1.01620000\end{array}$

C $-4.06771200 \quad 1.34659000 \quad 0.64896800$

C - $2.58750100-2.16263100 \quad 0.58323000$

C - $1.49963700 \quad 1.69697900 \quad 0.76386200$

$\begin{array}{lll}\text { C }-0.51037700 & -3.52985500 & -0.12801100\end{array}$

H -3.03150200 $3.07907600 \quad 1.36144700$

$\begin{array}{lll}H-1.41116000 & -3.40584500 & 1.84973000\end{array}$

H -3.93506300 $0.74024200 \quad 1.54490500$

H -5.03147900 $1.86401700 \quad 0.70868300$

$\begin{array}{lll}H-3.25037400 & -2.99008400 & 0.33009000\end{array}$

H -3.03010500 $-1.55254300 \quad 1.37316200$

$\begin{array}{lll}H-3.35422200 & 4.00278400 & -0.70736300\end{array}$

H -3.27830500 $2.53501100-1.51060200$

H $0.15640000 \quad-1.89074600 \quad 2.38557800$

H $-0.67739600 \quad-0.67654200 \quad 1.55748500$

H - $1.85190400 \quad 3.24259600 \quad-0.95933200$

H $0.50147000-1.65690000 \quad 0.75752400$

$\begin{array}{llll}\text { Ag } 1.55845700 & 1.23053800 & -0.06824500\end{array}$

Ag $3.85128500-0.05160000-0.14379700$ $\mathrm{H}=-957.810275$ Hartree

$\mathrm{G}=-957.886250$ Hartree

Structure 22

$\begin{array}{lrr}\text { S } 1.99979100 & 0.17345600 & -1.46158300 \\ \text { S } 4.11256900 & -0.20915500 & -2.23088900 \\ \text { O -0.83259300 } & 2.71078400 & -0.63128400 \\ \text { O 5.19142100 } & -1.04277600 & 2.44089900 \\ \text { O -0.71395600 } & 0.80491100 & 0.67771300 \\ \text { O } 4.14264100 & 0.58019000 & 1.17582500 \\ \text { N } 1.66781300 & 1.30840600 & 1.64432600 \\ \text { N } 6.73498200 & -1.91291800 & 0.60760400 \\ \text { C } 1.26291300 & 2.17409100 & 0.46688000 \\ \text { C } 5.74801500 & -0.86040100 & 0.09092500 \\ \text { C } 2.17697300 & 1.94803100 & -0.74979200 \\ \text { C } 4.84618500 & -1.46818700 & -1.00803600 \\ \text { C -0.23480200 } & 1.89599500 & 0.13609400 \\ \text { C } 4.95675800 & -0.39597500 & 1.35517300 \\ \text { H } 1.36220500 & 3.22450400 & 0.75615700 \\ \text { H } 6.32279200 & -0.02459400 & -0.31254100 \\ \text { H } 1.90132800 & 2.64759700 & -1.53943500 \\ \text { H } 3.22621400 & 2.06416600 & -0.47728700 \\ \text { H } 5.44007800 & -2.10958400 & -1.66881800 \\ \text { H } 4.02624300 & -2.05887700 & -0.59312300 \\ \text { H } 0.99873600 & 0.51653000 & 1.65397600 \\ \text { H } 1.55793900 & 1.82156200 & 2.52535000 \\ \text { H } 7.70765800 & -1.59335800 & 0.54488000\end{array}$




\author{
H $6.65826200-2.81487100 \quad 0.12479500$ \\ H $2.68005400 \quad 0.96731600 \quad 1.56255000$ \\ H $6.45755700-2.01838100 \quad 1.62007700$ \\ $\begin{array}{lll}\text { Ag }-2.83274500 & 0.05943700 & 0.27580900\end{array}$ \\ Ag $-5.24795200-0.91061700-0.10417600$ \\ $\mathrm{H}=-957.808263$ Hartree \\ $\mathrm{G}=-957.886561$ Hartree
}

\section{CYSTINE-Ag 4}

Structure 1

\begin{tabular}{|c|c|c|}
\hline 5.21048400 & 1.88363800 & -0.78525900 \\
\hline S 6.24115800 & 1.80666800 & 1.25474600 \\
\hline 0.83764400 & -0.94916700 & -0.35584700 \\
\hline O 7.56351500 & -1.02541500 & -1.73558800 \\
\hline O 0.90635100 & 0.92678000 & 0.99372800 \\
\hline O 5.64414400 & -1.66659500 & -0.61202400 \\
\hline 3.27372900 & -0.73806100 & -1.26188200 \\
\hline N 8.75949600 & -0.00689000 & 0.32121900 \\
\hline C 2.95066700 & 0.19103300 & -0.10950600 \\
\hline C 7.49861100 & -0.78082100 & 0.66860000 \\
\hline C 3.36708100 & 1.64226500 & -0.36988100 \\
\hline C 6.57205200 & -0.03481100 & 1.63639400 \\
\hline C 1.42467900 & 0.07402700 & 0.21104900 \\
\hline C 6.83515600 & -1.18239100 & -0.69261100 \\
\hline H 3.48410600 & -0.19967800 & 0.76292300 \\
\hline H 7.80834500 & -1.71245600 & 1.15512900 \\
\hline H 2.85419800 & 2.06590100 & -1.24167400 \\
\hline Н 3.12775400 & 2.25091200 & 0.50172700 \\
\hline H 5.62786900 & -0.57411300 & 1.69394100 \\
\hline H 7.00908400 & 0.02672900 & 2.63945000 \\
\hline H 2.50683400 & -1.43739300 & -1.27145500 \\
\hline H 4.23085200 & -1.19106800 & -1.11749800 \\
\hline H 9.57010500 & -0.28261100 & 0.88463800 \\
\hline H 8.60496900 & 1.00764000 & 0.42705000 \\
\hline H 3.26419400 & -0.25031200 & -2.16452500 \\
\hline H 8.91417400 & -0.22047600 & -0.69311400 \\
\hline g - -1.37410000 & $0-1.35353000$ & $0-0.03356600$ \\
\hline $\mathrm{Ag}-3.68404500$ & 0.31071800 & 0.02320000 \\
\hline $\mathrm{Ag}-3.79088800$ & $0-2.48023500$ & $0 \quad 0.24561500$ \\
\hline $\mathrm{Ag}-4.46935000$ & 0 2.86232600 & -0.16908200 \\
\hline \multicolumn{3}{|c|}{$H=-1249.418764$ Hartree } \\
\hline \multicolumn{3}{|c|}{$G=-1249.512230$ Hartree } \\
\hline
\end{tabular}

Structure 2

\begin{tabular}{|c|c|c|}
\hline 5300 & -2.6 & \\
\hline S -4.82607800 & -1.40626500 & 1.41914000 \\
\hline O -5.84996300 & 1.30615200 & -0.68132800 \\
\hline O -1.75666100 & 2.73638300 & 0.64115200 \\
\hline O -7.71826500 & 0.89176600 & 0.62249700 \\
\hline O -0.72974000 & 0.67957200 & 0.89147400 \\
\hline N -6.39993100 & -0.37052300 & -2.54339800 \\
\hline N -4.15615100 & 1.92504000 & 1.25609300 \\
\hline C - 7.35672700 & -0.45478500 & -1.36107900 \\
\hline C -3.09388100 & 0.84992900 & 1.39147000 \\
\hline C - 7.36224900 & -1.84154500 & -0.69267500 \\
\hline C -3.40868300 & -0.41682700 & 0.58380900 \\
\hline C -6.96395500 & 0.68197900 & -0.36863100 \\
\hline C - 1.73733700 & 1.48738800 & 0.92323300 \\
\hline$H-8.36843000$ & -0.25474900 & -1.72562400 \\
\hline H -2.97657500 & 0.60967800 & 2.45351600 \\
\hline H -7.92504600 & -1.78284600 & 0.23961300 \\
\hline H -7.82637400 & -2.59158100 & -1.34275400 \\
\hline H -3.71509100 & -0.17335600 & -0.43403000 \\
\hline H -2.52602500 & -1.05415100 & 0.57102500 \\
\hline H -5.79089500 & 0.45213000 & -2.31206600 \\
\hline H -6.88541400 & -0.22724300 & -3.43486300 \\
\hline H -4.68082600 & 2.05966400 & 2.12517200 \\
\hline H -4.86057400 & 1.72674300 & 0.46339100 \\
\hline H -5.80300300 & -1.20669200 & -2.61380200 \\
\hline H -3.62204800 & 2.79131000 & 1.02915900 \\
\hline Ag 1.36745900 & 1.26840200 & 0.24105100 \\
\hline Ag 3.68843100 & 2.31534900 & -0.59318300 \\
\hline Ag 3.61513800 & -0.44914600 & -0.12628200 \\
\hline Ag 4.40521500 & -2.99690600 & 0.09707000 \\
\hline \multicolumn{3}{|c|}{$\mathrm{H}=-1249.417145$ Hartree } \\
\hline \multicolumn{3}{|c|}{$G=-1249.511839$ Hartree } \\
\hline
\end{tabular}

Structure 3

$\begin{array}{lrl}\text { S } 2.35883900 & -1.31759600 & 2.07431300 \\ \text { S } 4.06146400 & 0.19143900 & 1.76078100 \\ \text { O } 1.29021400 & -1.46933900 & -1.36563200 \\ \text { O } 4.34250800 & -0.20209000 & -1.72588100 \\ \text { O } 0.24774700 & -3.08868700 & -0.11034000 \\ \text { O } 5.58966200 & 1.66620200 & -1.21888200 \\ \text { N } 3.72397700 & -2.66201400 & -1.19347800 \\ \text { N } 1.98415900 & 1.11592800 & -1.28757400 \\ \text { C } 2.64480700 & -3.14601300 & -0.24526700 \\ \text { C } 3.24404100 & 1.69076100 & -0.67156300\end{array}$




$\begin{array}{lll}\text { C } 3.00029800 & -2.90891800 & 1.23977400 \\ \text { C } 3.22066300 & 1.65815500 & 0.87261300 \\ \text { C } 1.28442600 & -2.51548400 & -0.62089800 \\ \text { C } 4.50573500 & 1.00140700 & -1.26163500 \\ \text { H 2.56987400 } & -4.22891800 & -0.38760600 \\ \text { H 3.26906800 } & 2.74569400 & -0.96218900 \\ \text { H 2.51687100 } & -3.68503600 & 1.83886500 \\ \text { H 4.07755500 } & -2.97003100 & 1.41473800 \\ \text { H 3.80651400 } & 2.50255900 & 1.24674800 \\ \text { H 2.20353100 } & 1.75512700 & 1.26372300 \\ \text { H 3.83900600 } & -1.58978300 & -1.27435700 \\ \text { H 3.51157800 } & -2.98832700 & -2.14414000 \\ \text { H } 1.96461100 & 1.33984700 & -2.29008000 \\ \text { H } 1.13293800 & 1.54873400 & -0.85933400 \\ \text { H 4.63368500 } & -3.05772600 & -0.92804900 \\ \text { H } 1.89640800 & 0.06454700 & -1.21988200 \\ \text { Ag -1.69033200 } & -1.83799000 & -0.11869800 \\ \text { Ag -4.07884400 } & -0.62853700 & -0.03141800 \\ \text { Ag -1.81016300 } & 1.01037200 & -0.00114200 \\ \text { Ag -0.44756100 } & 3.31735200 & 0.14690000 \\ \text { H= -1249.421173 Hartree } & \\ \text { G= -1249.511194 Hartree } & \end{array}$

Structure 4

$\begin{array}{lrc}\text { S 7.41119500 } & -0.88576400 & 0.13278900 \\ \text { S 5.93026500 } & -0.55206800 & -1.58586000 \\ \text { O } 4.44151100 & 1.56382600 & 0.90958500 \\ \text { O } 0.79009100 & -0.43212400 & -0.76177700 \\ \text { O } 5.86644500 & 2.99323900 & -0.22448400 \\ \text { O } 1.71743500 & -2.50678300 & -1.18740600 \\ \text { N } 6.15343900 & 0.73979300 & 2.63733600 \\ \text { N } 2.92552200 & 0.95796000 & -1.17677200 \\ \text { C } 6.77036900 & 1.60828600 & 1.54833300 \\ \text { C } 3.08696400 & -0.51367200 & -1.51107000 \\ \text { C 7.84969700 } & 0.87021100 & 0.73596500 \\ \text { C } 4.28636500 & -1.16336000 & -0.80711200 \\ \text { C 5.60350300 } & 2.12338500 & 0.65291500 \\ \text { C } 1.75469400 & -1.24276800 & -1.11450600 \\ \text { H 7.24364800 } & 2.47181700 & 2.02405100 \\ \text { H } 3.18246200 & -0.61034200 & -2.59802400 \\ \text { H } 8.12538200 & 1.48134800 & -0.12425400 \\ \text { H } 8.74350400 & 0.68749100 & 1.34289400 \\ \text { H } 4.30570500 & -0.91914100 & 0.25549500 \\ \text { H } 4.23525300 & -2.24221900 & -0.94681900 \\ \text { H } 5.12662600 & 0.76376900 & 2.42116800\end{array}$

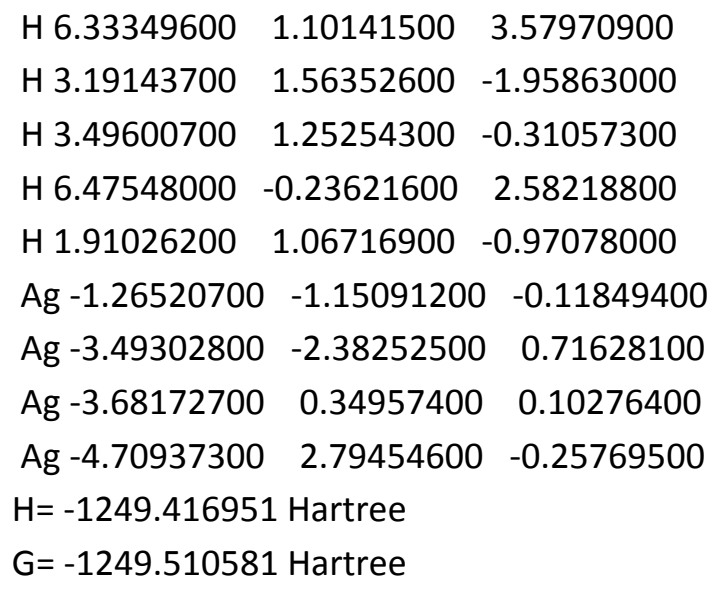


Structure 6

\begin{tabular}{|c|c|c|}
\hline S -6.32265200 & 1.65024700 & -0.99952100 \\
\hline S -5.45066700 & -0.32576700 & -1.77022800 \\
\hline O -6.47463500 & -1.57471500 & 1.67012400 \\
\hline O -0.67702500 & -1.87322500 & -0.38902600 \\
\hline O -4.41199500 & -0.52331500 & 1.66214000 \\
\hline O - 0.81438600 & -0.53672700 & -2.27026500 \\
\hline N -5.28405800 & 1.85655900 & 2.06659300 \\
\hline N -3.21635100 & -2.17273800 & -0.02981800 \\
\hline C -6.39585600 & 0.84334300 & 1.82759600 \\
\hline C - 2.86964500 & -1.43458300 & -1.30974600 \\
\hline C - 7.25695900 & 1.17960200 & 0.59646600 \\
\hline C - 3.57726000 & -0.07804700 & -1.43536300 \\
\hline C - 5.72506300 & -0.55897300 & 1.71518800 \\
\hline C - 1.31241800 & -1.24316700 & -1.34434900 \\
\hline H -7.05166600 & 0.84053700 & 2.70288200 \\
\hline H -3.12515800 & -2.07879600 & -2.15812400 \\
\hline H -7.91660400 & 0.33716500 & 0.38530700 \\
\hline H -7.86635100 & 2.07288600 & 0.77467200 \\
\hline H -3.47256000 & 0.51316000 & -0.52528700 \\
\hline H -3.15786900 & 0.46012200 & -2.28424000 \\
\hline H -4.41190700 & 1.27246400 & 2.07272500 \\
\hline H -5.39079400 & 2.35776800 & 2.95441900 \\
\hline H -2.29854600 & -2.50107300 & 0.33724200 \\
\hline H -3.83247300 & -2.97282100 & -0.20023200 \\
\hline H -5.21409700 & 2.53969100 & 1.29976100 \\
\hline H -3.68151000 & -1.53659900 & 0.70636600 \\
\hline Ag 1.56066300 & -1.62968600 & -0.08391400 \\
\hline Ag 3.20505100 & 0.68870700 & 0.09512000 \\
\hline Ag 4.16731900 & -1.91820100 & 0.46526500 \\
\hline Ag 3.13384000 & 3.35985800 & -0.08688500 \\
\hline \multicolumn{3}{|c|}{$H=-1249.417064$ Hartree } \\
\hline \multicolumn{3}{|c|}{$G=-1249.510244$ Hartree } \\
\hline
\end{tabular}

Structure 7

$\begin{array}{lll}\text { S 6.46704400 } & 1.93241200 & 0.68362500 \\ \text { S 5.45103200 } & 0.23763100 & 1.84702000 \\ \text { O } 6.74538900 & -1.88691300 & -1.01335500 \\ \text { O } 0.76644500 & -1.57981300 & 0.48770500 \\ \text { O } 4.72833000 & -0.85711700 & -1.49452300 \\ \text { O } 0.79335000 & 0.19866400 & 1.96474600 \\ \text { N } 5.71542300 & 1.33101100 & -2.40543800 \\ \text { N } 3.31736500 & -1.98800000 & 0.44117700 \\ \text { C } 6.76484700 & 0.40333300 & -1.80724800\end{array}$

$\begin{array}{lrc}\text { C } 2.89065800 & -0.93277300 & 1.44550900 \\ \text { C } 7.52543800 & 1.04315300 & -0.63175300 \\ \text { C } 3.62876400 & 0.40136900 & 1.26793800 \\ \text { C } 6.03832400 & -0.91395300 & -1.39958100 \\ \text { C } 1.34230500 & -0.72691700 & 1.29644100 \\ \text { H } 7.49406100 & 0.16374500 & -2.58617700 \\ \text { H 3.05486600 } & -1.33018700 & 2.45323600 \\ \text { H } 8.13000400 & 0.27842600 & -0.14274100 \\ \text { H } 8.18194900 & 1.84873000 & -0.97884500 \\ \text { H 3.63154600 } & 0.71824000 & 0.22459600 \\ \text { H 3.14954900 } & 1.15486700 & 1.89141600 \\ \text { H 4.82994600 } & 0.77011100 & -2.35718800 \\ \text { H 5.92702200 } & 1.59440100 & -3.37340700 \\ \text { H 2.42245200 } & -2.38858700 & 0.08954300 \\ \text { H 3.88524300 } & -2.72406000 & 0.87126100 \\ \text { H 5.59137400 } & 2.18348300 & -1.84174200 \\ \text { H 3.87011600 } & -1.57569000 & -0.38783200 \\ \text { Ag -1.47530600 } & -1.53198000 & 0.11660000 \\ \text { Ag -3.37698100 } & 0.57312900 & -0.14350200 \\ \text { Ag -4.04590400 } & -2.14350600 & -0.32578400 \\ \text { Ag -3.60174500 } & 3.24152600 & -0.09810200 \\ \text { H= -1249.417023 Hartree } & \\ \text { G= -1249.510099 Hartree } & \end{array}$

Structure 8

\begin{tabular}{|c|c|c|}
\hline S - 4.52285800 & -0.51415500 & 1.19673600 \\
\hline S -4.66617200 & 0.44110600 & -0.87125600 \\
\hline O -0.86501600 & -3.32183100 & -0.41536300 \\
\hline O - 0.87824400 & 3.35540600 & 0.19958100 \\
\hline O - 2.35064200 & -4.99468100 & 0.13682100 \\
\hline O - 2.45544400 & 4.96708900 & -0.27477600 \\
\hline$N-4.49121600$ & -3.59491900 & 0.01156100 \\
\hline$N-4.53193000$ & 3.54752500 & 0.20430300 \\
\hline C -3.22851900 & -2.75660700 & -0.13247700 \\
\hline C - 3.24474400 & 2.73531700 & 0.22159900 \\
\hline C -3.07672000 & -1.74729100 & 1.01162600 \\
\hline C -3.21595700 & 1.68252600 & -0.89378800 \\
\hline C - 2.03459300 & -3.78032300 & -0.14049200 \\
\hline C - 2.08198900 & 3.77735300 & 0.03522500 \\
\hline H -3.26262000 & -2.23225000 & -1.09049400 \\
\hline H -3.14842800 & 2.25061200 & 1.19596700 \\
\hline H -3.04417700 & -2.23222900 & 1.99461600 \\
\hline H -2.15709000 & -1.17661700 & 0.87042200 \\
\hline H -3.29919300 & 2.12969200 & -1.89149800 \\
\hline - -2.28104500 & 1.12109200 & -0.83738800 \\
\hline
\end{tabular}




$$
\begin{array}{ccc}
\text { H }-5.00492800 & -3.66885300 & -0.87368400 \\
\text { H-5.13285100 } & -3.22529700 & 0.72382400 \\
\text { H-4.91044500 } & 3.67916800 & 1.14905000 \\
\text { H-5.26261000 } & 3.11790400 & -0.37688000 \\
\text { H-4.12512400 } & -4.54591400 & 0.27235600 \\
\text { H-4.23034600 } & 4.48166900 & -0.17100100 \\
\text { Ag } 0.27946200 & -1.30722200 & -0.31710600 \\
\text { Ag 2.73746800 } & 0.00786600 & -0.01003100 \\
\text { Ag 0.27638500 } & 1.35198100 & 0.07808500 \\
\text { Ag 5.42445800 } & -0.00394700 & 0.13279000 \\
\text { H= -1249.415354 Hartree } & \\
\text { G= -1249.508972 Hartree } &
\end{array}
$$

Structure 9

$$
\begin{aligned}
& \text { S } 5.46144000 \quad-2.03113100 \quad 1.80511400 \\
& \text { S } 6.34708700-2.09158900-0.29581600 \\
& \begin{array}{llll}
0 & 1.17000300 & -0.43744000 & 2.29753400
\end{array} \\
& \begin{array}{llll}
0 & 6.53915600 & 2.67794200 & -0.72684300
\end{array} \\
& \begin{array}{llll}
0 & 0.88359800 & -0.04605300 & 0.03732900
\end{array} \\
& \begin{array}{llll}
0 & 4.92605000 & 1.04053800 & -0.70539300
\end{array} \\
& \text { N } 3.18715700-0.94822600-0.71465100 \\
& \text { N } 8.431601001 .29604200-1.69740800 \\
& \text { C } 2.86325400 \quad-1.24325900 \quad 0.73934600 \\
& \begin{array}{llll}
\text { C } 7.19928800 & 0.44458400 & -1.37425300
\end{array} \\
& \text { C } 3.99011600-0.82822100 \quad 1.69023700 \\
& \text { C } 7.57391000-0.63281600-0.32846400 \\
& \text { C } 1.52555900-0.502634001 .08274300 \\
& \begin{array}{lll}
\text { C } 6.11997800 & 1.47271000 & -0.89219800
\end{array} \\
& \text { H } 2.66230200 \quad-2.31555500 \quad 0.83474100 \\
& \text { H } 6.86591200-0.03123200-2.29931100 \\
& \text { H } 4.37169400 \quad 0.16647200 \quad 1.45608200 \\
& \text { H } 3.60004700 \quad-0.84007800 \quad 2.71315100 \\
& \text { H } 8.53054100-1.09363100-0.59279700 \\
& \text { H } 7.64055900 \quad-0.22613900 \quad 0.68309300 \\
& \text { H } 3.51691500-1.77771200-1.21593900 \\
& \text { H } 3.92847500-0.18517500-0.79517100 \\
& \text { H } 8.62537800 \quad 1.32778600-2.70411900 \\
& \text { H } 9.280447000 .98433500-1.21282600 \\
& \text { H } 2.29443300-0.60249000-1.12102900 \\
& \text { H } 8.14523500 \quad 2.25407300-1.35573300 \\
& \text { Ag -1.14536800 } 0.95339600 \quad 0.20899000 \\
& \text { Ag -3.75220800 }-0.13220600-0.16792000 \\
& \begin{array}{lll}
\text { Ag }-3.26706100 & 2.58486200 & 0.32183100
\end{array} \\
& \text { Ag -5.10159200 }-2.39060300 \quad-0.66885900 \\
& H=-1249.414702 \text { Hartree }
\end{aligned}
$$

$G=-1249.508859$ Hartree

Structure 10

S $3.85804500-0.54588300-2.47105700$

S $3.01571500-1.77653500-0.73234300$

$\begin{array}{llll}0 & 6.06209400 & -0.50248700 & 1.25353900\end{array}$

$\begin{array}{llll}0 & 1.50712200 & -3.21918200 & 2.12905100\end{array}$

$\begin{array}{llll}0 & 4.13743500 & 0.78236100 & 1.20906700\end{array}$

$\begin{array}{llll}0 & -0.39837500 & -2.87250700 & 0.88655000\end{array}$

N $6.78500600 \quad 0.01503200-1.17754100$

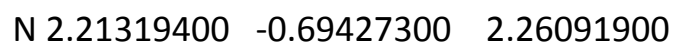

C $5.66189900 \quad 0.90362100-0.67098400$

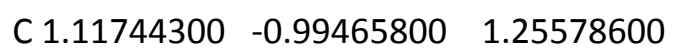

C $4.50877100 \quad 1.05615800-1.66883600$

$\begin{array}{llll}C & 1.54392600 & -0.67824500 & -0.18848900\end{array}$

C $5.24561700 \quad 0.33140400 \quad 0.72423400$

C $0.70314500-2.48934400 \quad 1.44674200$

H $6.08467800 \quad 1.89879900-0.49388700$

H $0.25844000-0.34910500 \quad 1.47119300$

H $3.685938001 .56592600-1.16756000$

H $4.80868800 \quad 1.64289600-2.54392900$

H $0.70928000-0.86099700-0.86589400$

H $1.86010800 \quad 0.36422700-0.26469600$

H $7.55091800 \quad 0.54096700-1.61042000$

H $6.42463700-0.67572000-1.85385400$

H $1.82628300-0.21804500 \quad 3.08310300$

H $3.01709300-0.095150001 .87018700$

H $7.11253600-0.49301300-0.32222100$

H $2.56656700-1.62432500 \quad 2.56415200$

Ag -2.19121100 - $-1.70042500 \quad 0.08024600$

Ag -2.12808600 $1.14411400 \quad 0.00727400$

Ag $-4.34761000 \quad-0.38399300-0.80290500$

Ag $-0.31232200 \quad 3.02408800 \quad 0.60024000$

$\mathrm{H}=-1249.417766$ Hartree

$G=-1249.508754$ Hartree

Structure 11

S $5.15890700 \quad 0.49468100-0.10414100$

S $3.75485000 \quad 1.50559300 \quad 1.38851600$

$\begin{array}{llll}0 & 5.13285900 & -3.19732500 & 0.08046600\end{array}$

$\begin{array}{llll}0 & 0.21294500 & 3.13446600 & -0.36912500\end{array}$

$\begin{array}{llll}0 & 3.77235900 & -2.40945500 & 1.78614700\end{array}$

$\begin{array}{llll}0 & 1.30363000 & 1.15081900 & -0.76890200\end{array}$

$\begin{array}{lll}\text { N } 2.03687000 & -1.25655000 & 0.22277200\end{array}$

N 2.23783300 $4.63437100-1.06007400$ 


$\begin{array}{ccc}\text { C } 3.27736900 & -1.75303100 & -0.49106100 \\ \text { C } 2.60451300 & 3.19189300 & -0.70400200 \\ \text { C } 4.04114000 & -0.61556600 & -1.19004800 \\ \text { C } 3.40826700 & 3.20142700 & 0.61606900 \\ \text { C } 4.14620000 & -2.53346400 & 0.55153100 \\ \text { C } 1.26588100 & 2.41464600 & -0.61130400 \\ \text { H } 2.96185600 & -2.45275300 & -1.27251500 \\ \text { H } 3.21331400 & 2.78372800 & -1.51289300 \\ \text { H } 4.77902300 & -1.05171500 & -1.87114700 \\ \text { H } 3.36265800 & 0.02685500 & -1.75294200 \\ \text { H } 4.36946600 & 3.69829600 & 0.46380000 \\ \text { H 2.85736000 } & 3.71731200 & 1.41147900 \\ \text { H } 1.75879300 & -0.29117500 & -0.07367500 \\ \text { H 2.27702500 } & -1.31273400 & 1.23468000 \\ \text { H 2.21699900 } & 4.77154000 & -2.07809400 \\ \text { H } 2.88264600 & 5.32540300 & -0.65821000 \\ \text { H } 1.21844200 & -1.88067400 & 0.05270400 \\ \text { H } 1.27320400 & 4.77401300 & -0.69304100 \\ \text { Ag }-1.70966700 & 1.84412800 & -0.04570400 \\ \text { Ag }-1.94860700 & -1.00778900 & -0.04322500 \\ \text { Ag }-4.09932700 & 0.72818700 & 0.42774100 \\ \text { Ag -0.78598200 } & -3.40752000 & -0.39827600 \\ \text { H= -1249.413942 Hartree } & \\ \text { G= -1249.508342 Hartree } & \end{array}$

Structure 12

\begin{tabular}{|c|c|c|}
\hline S -6.28155900 & -2.27451000 & 0.54058100 \\
\hline S -4.90795300 & -2.68690600 & -1.24064400 \\
\hline O -7.11939000 & 2.88815000 & 0.30874600 \\
\hline O -0.97304800 & 0.59294300 & -0.62179900 \\
\hline O -5.43826100 & 1.77395500 & -0.81210600 \\
\hline O -1.07689900 & -0.96660700 & 1.08355700 \\
\hline N -7.75629300 & 0.98688600 & 1.90467400 \\
\hline N -3.37797400 & 0.23610600 & -1.51148500 \\
\hline C -6.68041000 & 0.58003400 & 0.90273500 \\
\hline C -3.02643600 & -0.64977600 & -0.33338500 \\
\hline C -7.09435600 & -0.61720600 & 0.02226100 \\
\hline C -3.18692500 & -2.14750100 & -0.62193300 \\
\hline C -6.38691100 & 1.86754000 & 0.04940000 \\
\hline C -1.55801000 & -0.33011800 & 0.09887400 \\
\hline H -5.77694900 & 0.34600900 & 1.46922000 \\
\hline H -3.67715800 & -0.37130200 & 0.50117100 \\
\hline H -8.17372800 & -0.79256600 & 0.03179200 \\
\hline H -6.78948600 & -0.44606500 & -1.01088200 \\
\hline H -2.52661600 & -2.48210900 & -1.43126500 \\
\hline
\end{tabular}

$\begin{array}{lll}\text { H }-2.95192700 & -2.71236800 & 0.27988900 \\ \text { H-8.60215200 } & 0.40917400 & 1.85367300 \\ \text { H-7.97778300 } & 1.97901300 & 1.62195300 \\ \text { H-4.20024200 } & 0.86935700 & -1.26674800 \\ \text { H-3.62013800 } & -0.30497100 & -2.34886300 \\ \text { H-7.41424200 } & 0.97336000 & 2.87159900 \\ \text { H -2.51842600 } & 0.79710500 & -1.68905600 \\ \text { Ag } 1.16998400 & 1.23715000 & -0.22987300 \\ \text { Ag 3.46223300 } & 2.60280600 & 0.00690800 \\ \text { Ag 3.62197600 } & -0.19510800 & 0.08367200 \\ \text { Ag 4.63218900 } & -2.66545000 & 0.29230000 \\ \text { H=-1249.414908 Hartree } & \\ \text { G=-1249.508460 Hartree } & \end{array}$

Structure 13

S $4.13044800-2.40270500-1.10387000$ S $3.53717400-1.12945700 \quad 0.70268600$

$\begin{array}{llll}0 & 7.24521700 & -0.14660000 & 0.86773400\end{array}$

$\begin{array}{llll}0 & 2.64393600 & 1.27480100 & 3.16415100\end{array}$

$\begin{array}{llll}0 & 5.81504800 & 1.07027900 & -0.48625900\end{array}$

$\begin{array}{llll}0 & 0.61890000 & 1.01552200 & 2.10289100\end{array}$

N $7.35728900-2.22924000-0.66849700$

N $3.95815400 \quad 2.123962001 .06303000$

C $6.70831400-1.02022500-1.32105600$

C $2.58149600 \quad 1.56349900 \quad 0.75990800$

C $5.40376700-1.34918900-2.05399800$

C $2.63635800 \quad 0.32750300 \quad-0.15661000$

$\begin{array}{llll}\text { C } 6.57059200 & 0.06343400 & -0.20105700\end{array}$

$\begin{array}{lll}\text { C } 1.88673500 & 1.26473900 & 2.12989300\end{array}$

H $7.41534000 \quad-0.62613300-2.05934000$

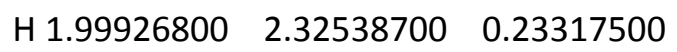

H $4.93738100-0.41314000-2.36070900$

H $5.58624800-1.96361900-2.94256900$

H $1.62356500 \quad 0.00551600-0.40111600$

H $3.187602000 .55831700-1.06998400$

H $8.08121900-2.66249700-1.25102100$

H $6.64688500-2.93794900-0.42888600$

H $3.96480800 \quad 3.14422500 \quad 0.95886400$

H $4.74671200 \quad 1.71477700 \quad 0.45300300$

$\begin{array}{lll}\text { H } 7.76465500 & -1.85066000 & 0.21898000\end{array}$

H $4.12406700 \quad 1.900302002 .06612500$

Ag-1.02997600 $1.25111000 \quad 0.54504200$

Ag -2.55419500 $2.06307800-1.50071900$

Ag -3.31955900 $-0.28226300-0.17002800$

Ag $-4.74044400 \quad-2.45221400 \quad 0.48974800$ 
$H=-1249.415082$ Hartree
$G=-1249.507602$ Hartree

Structure 14

S $3.07374800 \quad 0.11435000 \quad 1.04015500$

S $4.97564700 \quad 0.63357300 \quad 2.18395100$

$\begin{array}{llll}0 & 0.32204100 & -2.39822800 & 0.12228500\end{array}$

$\begin{array}{llll}0 & 7.41976700 & -0.03214700 & -2.02035700\end{array}$

$00.94006300-0.96971800-1.58825500$

O $5.76940000-1.02482000-0.74694600$

N $3.44686100-1.78922000-1.67936300$

N $8.35418300 \quad 1.48554100-0.20084400$

C $2.65508000 \quad-2.28672600-0.48643000$

$\begin{array}{lll}\text { C } 7.19582300 & 0.61699400 & 0.30246500\end{array}$

C $3.24398300-1.78793700 \quad 0.84470900$

C $6.07806900 \quad 1.50135500 \quad 0.89749300$

C $1.17350500-1.84533900-0.68261600$

C $6.75389300-0.22457000-0.93788600$

H $2.69472600 \quad-3.37969900-0.47057200$

H $7.59269300-0.05351500 \quad 1.06861100$

H $2.70156400-2.250301001 .66956500$

H $4.30947900 \quad-2.00965600 \quad 0.90935600$

H $6.51755600 \quad 2.31968900 \quad 1.47823700$

H $5.42933900 \quad 1.92522200 \quad 0.12769700$

H $2.89371100-1.01040000-2.08331800$

H $3.52294900-2.52073600-2.39390200$

H $9.21590900 \quad 1.33850900 \quad 0.33481000$

H $8.134774002 .48812900-0.19612900$

H $4.43077900-1.46826400-1.40377500$

H $8.474959001 .15954900-1.19725200$

Ag -1.85427000 $-1.71708900 \quad 0.11163500$

Ag -3.03631000 $0.87447600 \quad-0.05755400$

$\begin{array}{llll}\text { Ag }-4.52253600 & -1.48692600 & 0.24274200\end{array}$

Ag $-2.40663700 \quad 3.46096700 \quad-0.33996700$

$\mathrm{H}=-1249.413059$ Hartree

$\mathrm{G}=-1249.507681$ Hartree

Structure 15

$\begin{array}{lrc}\text { S } 2.91207300 & -0.13813800 & -1.37517900 \\ \text { S } 3.85846800 & -1.29045600 & 0.35428300 \\ \text { O } 0.45648400 & 2.37409300 & -0.30003100 \\ \text { O } 8.11990800 & 0.02959400 & 0.95882600 \\ \text { O } 1.09778300 & 1.18909500 & 1.58039400 \\ \text { O } 6.07320800 & 1.08255900 & 0.98219200 \\ \text { N } 3.62111300 & 1.93088400 & 1.48384000\end{array}$

N $7.80086300 \quad-1.21624100 \quad-1.24353600$

$\begin{array}{llll}\text { C } 2.79763300 & 2.31287700 & 0.26666400\end{array}$

C $6.49448400-0.63515300-0.69768700$

C $3.34408000 \quad 1.68921400-1.02982600$

C $5.54025000-1.79879600-0.35577600$

$\begin{array}{llll}\text { C } 1.31851900 & 1.92167700 & 0.55380600\end{array}$

$\begin{array}{lll}\text { C } 6.92542900 & 0.23904000 & 0.52801100\end{array}$

H $2.85251200 \quad 3.39893200 \quad 0.14768400$

H $6.04937300-0.00619800-1.47111100$

H $2.875346002 .18879500-1.88326100$

H $4.425881001 .81670800-1.09471600$

H $5.34138200-2.39526600-1.24996100$

H $5.95667100-2.44623200 \quad 0.42500200$

H $4.58003700 \quad 1.53721900 \quad 1.24036400$

H $3.05183600 \quad 1.23505400 \quad 2.00259800$

H $8.12283400-0.71452100-2.07925900$

H $7.74328600-2.21539100-1.46905000$

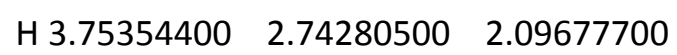

H $8.47884300-1.04617000-0.45437400$

Ag $-1.72675100 \quad 1.74001600-0.18240000$

Ag $-4.40115400 \quad 1.54966800 \quad-0.13280300$

$\begin{array}{lll}\text { Ag }-2.93968000 & -0.83405600 & 0.01963200\end{array}$

$\begin{array}{lll}\text { Ag }-2.33699900 & -3.43470400 & 0.23342900\end{array}$

$\mathrm{H}=-1249.413137$ Hartree

$G=-1249.507303$ Hartree

Structure 16

S $6.60553200-1.15906100 \quad 0.13304700$

S $4.99871700-2.47473100-0.82957300$

$\begin{array}{llll}0 & 7.05386900 & 2.74270400 & 1.29289700\end{array}$

$\begin{array}{llll}0 & 0.92437200 & 0.14554900 & 1.34927200\end{array}$

$\begin{array}{llll}0 & 5.15513700 & 2.06205900 & 0.16115000\end{array}$

$00.62131200-1.51209900-0.23303100$

N $8.740865001 .45401900-0.16774300$

N $3.51492800 \quad 0.241989001 .16176800$

$\begin{array}{llll}\text { C } 7.33041300 & 1.48789100 & -0.75144300\end{array}$

C $2.87133800 \quad-0.69628000 \quad 0.15940800$

C $6.83211500 \quad 0.13787900-1.26917500$

$\begin{array}{lll}\text { C } 3.43778600 & -2.11999300 & 0.20300800\end{array}$

$\begin{array}{lll}\text { C } 6.42621200 & 2.15123700 & 0.34255100\end{array}$

$\begin{array}{llll}\text { C } 1.33098500 & -0.68767600 & 0.46534800\end{array}$

H $7.359411002 .17354000-1.60593500$

H $2.99791200-0.26691700-0.83888400$

H $5.85267400 \quad 0.29291200-1.72142400$

H $7.51341800-0.28834300-2.00814700$ 


$\begin{array}{lll}\text { H } 3.61444700 & -2.46119500 & 1.22669100 \\ \text { H } 2.70992400 & -2.79029200 & -0.26646800 \\ \text { H } 8.68101400 & 2.12037800 & 0.64216800 \\ \text { H } 9.45470000 & 1.75707200 & -0.83843300 \\ \text { H } 4.12302100 & 0.99444000 & 0.71485200 \\ \text { H } 4.09711700 & -0.26054100 & 1.84041400 \\ \text { H } 9.00227900 & 0.52597600 & 0.18596000 \\ \text { H 2.70923400 } & 0.67756600 & 1.66345400 \\ \text { Ag -1.65318200 } & -1.52515900 & -0.05278200 \\ \text { Ag -4.26684600 } & -2.07286400 & 0.16218200 \\ \text { Ag -3.53152300 } & 0.62281800 & -0.07379000 \\ \text { Ag -3.71832700 } & 3.28690600 & -0.25408800 \\ \text { H= -1249.412786 Hartree } & \\ \text { G= -1249.507179 Hartree } & \end{array}$

Structure 17

$\begin{array}{lrc}\text { S } 6.28931400 & 1.40216800 & -1.45923300 \\ \text { S } 5.02142500 & 2.67526500 & -0.01012000 \\ \text { O } 7.57937100 & -2.55402100 & 0.50336800 \\ \text { O } 0.92048500 & -0.69590200 & -0.24879500 \\ \text { O } 5.56770600 & -1.74996800 & -0.26975500 \\ \text { O } 0.74040600 & 1.31314100 & 0.88092700 \\ \text { N } 8.25093800 & -0.47154300 & 1.82000800 \\ \text { N } 3.40410300 & -0.42926800 & -0.93342400 \\ \text { C } 7.10592800 & -0.21169300 & 0.83819500 \\ \text { C } 2.93003900 & 0.61159800 & 0.05952200 \\ \text { C 7.60570200 } & 0.75044700 & -0.26101000 \\ \text { C 3.25795900 } & 2.04851000 & -0.35443300 \\ \text { C } 6.70786000 & -1.62833200 & 0.30873900 \\ \text { C } 1.38395900 & 0.42073300 & 0.25164700 \\ \text { H } 6.27966100 & 0.24394400 & 1.38701400 \\ \text { H } 3.39398400 & 0.38255700 & 1.02508200 \\ \text { H } 8.07100900 & 1.62978600 & 0.19270200 \\ \text { H } 8.33511500 & 0.26823500 & -0.92172300 \\ \text { H 3.02465900 } & 2.23823300 & -1.40575400 \\ \text { H } 2.65159200 & 2.72423400 & 0.25766700 \\ \text { H } 8.56611200 & -1.44330500 & 1.56566500 \\ \text { H 7.92266200 } & -0.47008600 & 2.79269600 \\ \text { H 2.62457500 } & -1.11730500 & -0.98282600 \\ \text { H } 4.31718500 & -0.90916100 & -0.63409800 \\ \text { H } 9.02277200 & 0.19934000 & 1.73903100 \\ \text { H 3.53921100 } & -0.03006100 & -1.86807000 \\ \text { Ag -1.27204100 } & -1.26324900 & -0.11551300 \\ \text { Ag -3.59754700 } & -2.59495500 & -0.08292500 \\ \text { Ag -3.70366400 } & 0.20540700 & 0.03251800\end{array}$

Ag $-4.67606900 \quad 2.69315300 \quad 0.21218100$

$\mathrm{H}=-1249.413868$ Hartree

$\mathrm{G}=-1249.507130$ Hartree

Structure 18

\begin{tabular}{|c|c|c|}
\hline S 3.98502000 & 0.28961400 & 1.40620100 \\
\hline S 6.18575500 & 0.37985900 & 2.01302100 \\
\hline O 0.98598100 & -0.38450900 & -0.32762400 \\
\hline O 5.71441800 & -1.48209200 & -0.93343600 \\
\hline O 0.59351500 & -1.65878400 & 1.56295300 \\
\hline O 6.69590500 & -0.30590500 & -2.66250000 \\
\hline N 3.11320100 & -1.71862300 & -1.09162500 \\
\hline N 8.76313500 & 0.43979200 & -1.34572600 \\
\hline C 2.64613200 & -2.01468900 & 0.32006800 \\
\hline C 7.65982300 & -0.13305700 & -0.45125300 \\
\hline C 3.69695800 & -1.60882500 & 1.36809800 \\
\hline C 7.09509200 & 0.98445200 & 0.45692600 \\
\hline C 1.27765700 & -1.30898000 & 0.55346900 \\
\hline C 6.59716500 & -0.69858500 & -1.44356900 \\
\hline H 2.49491000 & -3.09383600 & 0.41763500 \\
\hline H 8.08544900 & -0.93447200 & 0.15455400 \\
\hline H 3.34925200 & -1.90760100 & 2.35738200 \\
\hline H 4.66420200 & -2.05899100 & 1.14369400 \\
\hline H 7.91663700 & 1.56629700 & 0.89115700 \\
\hline H 6.43535300 & 1.66534800 & -0.08614500 \\
\hline H 2.63870900 & -0.84084900 & -1.37046700 \\
\hline H 2.80903100 & -2.45595300 & -1.73626700 \\
\hline H 8.29928300 & 0.51707400 & -2.28590300 \\
\hline H 9.55897500 & -0.20399500 & -1.41985600 \\
\hline H 4.18328800 & -1.62040400 & -1.14702200 \\
\hline H 9.11337000 & 1.35233300 & -1.03505200 \\
\hline Ag -0.94813300 & $0 \quad 0.79226900$ & $0-0.18113200$ \\
\hline $\mathrm{Ag}-3.69180900$ & $0 \quad 0.02232200$ & $0-0.08723400$ \\
\hline $\mathrm{Ag}-2.83977100$ & $0 \quad 2.69100000$ & $0-0.08354800$ \\
\hline $\mathrm{Ag}-5.31115300$ & $0 \quad-2.11019300$ & $\begin{array}{ll}0 & -0.06228600\end{array}$ \\
\hline \multicolumn{3}{|c|}{$H=-1249.412704$ Hartree } \\
\hline \multicolumn{3}{|c|}{$G=-1249.507205$ Hartree } \\
\hline
\end{tabular}

Structure 19

$\begin{array}{lll}\text { S } 5.98892500 & 0.26679300 & 2.16182500\end{array}$

S $6.93600200-0.72027700 \quad 0.32957000$

$\begin{array}{llll}0 & 0.83891000 & -0.45186600 & 1.53222300\end{array}$

$\begin{array}{llll}0 & 4.49318400 & 1.38552900 & -3.70922700\end{array}$

O $2.42340700-0.73710200-0.11545100$

$\begin{array}{llll}0 & 5.76614500 & 2.94241400 & -2.55227100\end{array}$ 


$\begin{array}{ccc}\text { N } 2.35354100 & 0.70852700 & 3.24972300 \\ \text { N } 4.47238800 & -0.41484100 & -1.87621100 \\ \text { C } 3.09171200 & 0.30191400 & 1.97664300 \\ \text { C } 5.23370800 & 0.83099500 & -1.46949300 \\ \text { C } 4.29611700 & -0.62606600 & 2.24794100 \\ \text { C } 6.68403600 & 0.56117200 & -1.06358200 \\ \text { C } 2.03293500 & -0.36444500 & 1.03787800 \\ \text { C 5.16652600 } & 1.82433700 & -2.69204200 \\ \text { H } 3.42102300 & 1.22039300 & 1.48464100 \\ \text { H } 4.70104900 & 1.29638300 & -0.63476200 \\ \text { H } 4.24766900 & -1.09343800 & 3.23537500 \\ \text { H 4.33611400 } & -1.41838300 & 1.49950400 \\ \text { H 7.26872800 } & 0.12993000 & -1.88561300 \\ \text { H 7.14925400 } & 1.49749500 & -0.75657200 \\ \text { H 2.73200300 } & 0.26213700 & 4.09254700 \\ \text { H } 1.36977900 & 0.38114400 & 3.09237300 \\ \text { H } 3.65870800 & -0.58628300 & -1.22951200 \\ \text { H } 5.06260200 & -1.25329800 & -1.89259900 \\ \text { H 2.35779800 } & 1.72424400 & 3.39471700 \\ \text { H 4.14560900 } & -0.18117100 & -2.84736600 \\ \text { Ag -1.01823700 } & -1.16400800 & 0.37319400 \\ \text { Ag -3.46628100 } & 0.22028300 & -0.09517700 \\ \text { Ag -2.98191600 } & -2.40689200 & -0.95559200 \\ \text { Ag -4.70875800 } & 2.56213900 & 0.28404500 \\ \text { H= -1249.410491 Hartree } & \\ \text { G=-1249.507008 Hartree } & & \\ & & \\ \end{array}$

CYSTINE-Au

Structure 1

$\begin{array}{ccc}\text { S } 4.51010000 & -0.57599900 & 1.75602400 \\ \text { S 4.98958700 } & -2.14999600 & 0.16717700 \\ \text { O } 0.42394200 & 2.21022900 & 0.05014700 \\ \text { O } 7.26866400 & 1.52547100 & 0.04458700 \\ \text { O } 0.05422300 & -0.05900300 & 0.30930800 \\ \text { O } 5.18030400 & 1.67062900 & -0.94267500 \\ \text { N } 3.03219300 & 2.06221300 & 0.49110100 \\ \text { N } 7.80612300 & -0.86119400 & -0.80216700 \\ \text { C } 2.32564600 & 0.72898800 & 0.36331200 \\ \text { C } 6.56329600 & -0.25454500 & -1.43122300 \\ \text { C } 2.65179300 & -0.23589900 & 1.50996300 \\ \text { C } 5.36115700 & -1.20654500 & -1.45042600 \\ \text { C } 0.79886200 & 1.01129700 & 0.23908900 \\ \text { C } 6.31043700 & 1.10313200 & -0.69327100\end{array}$

$\begin{array}{lcc}\text { H } 2.64525600 & 0.29599100 & -0.58953700 \\ \text { H } 6.80727800 & -0.00739500 & -2.47049700 \\ \text { H 2.33541600 } & 0.16123200 & 2.48160300 \\ \text { H 2.14614700 } & -1.18606300 & 1.34144400 \\ \text { H } 4.48107400 & -0.64400500 & -1.75781900 \\ \text { H } 5.51858800 & -2.03293100 & -2.15261000 \\ \text { H } 2.37665600 & 2.77562600 & 0.12348700 \\ \text { H } 3.95049600 & 2.04349900 & -0.06322100 \\ \text { H } 8.46278200 & -1.24227300 & -1.49090800 \\ \text { H } 7.55015800 & -1.60320400 & -0.13259800 \\ \text { H } 3.23492700 & 2.29846100 & 1.46882400 \\ \text { H } 8.23555600 & -0.06482000 & -0.27346500 \\ \text { Au -2.09520600 } & -0.03686600 & 0.06567000 \\ \text { Au -4.65279100 } & -0.15607700 & -0.21749400 \\ \text { H= -937.207950 Hartree } & \\ \text { G= -937.284531 Hartree } & \end{array}$

Structure 2

S - $4.92040400 \quad 1.37736400 \quad 1.22341900$

S -5.88385500 $1.66845900-0.82981700$

O $-0.00277300 \quad-0.16097000 \quad 0.36390200$

$\begin{array}{lll}0-6.54207400 & -2.11914500 & 1.13080000\end{array}$

$\begin{array}{llll}0 & -0.48373000 & 1.97932700 & -0.37085600\end{array}$

$\begin{array}{llll}0 & -4.49676200 & -1.94073500 & 0.06261300\end{array}$

N-2.42185900 $-0.75531900 \quad 1.12566900$

N-7.90693200 $-0.87303200-0.68425300$

$\begin{array}{llll}\text { C }-2.31014700 & 0.50789300 & 0.29721900\end{array}$

C $-6.48144200-1.19240800-1.10124700$

C $-3.07048000 \quad 1.69311000 \quad 0.90489800$

$\begin{array}{llll}C-5.75328900 & -0.00464700 & -1.74069500\end{array}$

C $-0.80529000 \quad 0.84591700 \quad 0.07744700$

C - $5.77345600 \quad-1.79490200 \quad 0.15859500$

H-2.72101100 $0.26754600 \quad-0.68856400$

H -6.52991100 $-1.99322000-1.84754900$

H - $2.68405400 \quad 1.95984000 \quad 1.89567400$

$\begin{array}{lll}H-2.97527300 & 2.56005100 & 0.25156600\end{array}$

$\begin{array}{lll}H-4.70565300 & -0.27135200 & -1.87118600\end{array}$

$\begin{array}{lll}H-6.18094100 & 0.24558900 & -2.71807500\end{array}$

H - $1.53029100-1.26465100 \quad 0.99226900$

H -3.26058300 $-1.33649000 \quad 0.79721800$

$\begin{array}{lll}H-8.60279400 & -1.15240600 & -1.38332300\end{array}$

$\mathrm{H}-8.01252300 \quad 0.13396300-0.48654600$

$\begin{array}{lll}H-2.51912100 & -0.55107500 & 2.12662600\end{array}$

$\begin{array}{lll}H-8.03416600 & -1.40503400 & 0.20900800\end{array}$

$\begin{array}{lll}\text { Au } 2.13999500 & -0.08644400 & 0.09324600\end{array}$ 
Au $4.69916100 \quad-0.12779700 \quad-0.19114000$ $\mathrm{H}=-937.207556$ Hartree

$G=-937.284165$ Hartree

Structure 3

\begin{tabular}{|c|c|c|}
\hline S -4.90088500 & -2.17969500 & 0.79042400 \\
\hline S -3.21746700 & -0.86995300 & -0.03652100 \\
\hline O -5.93389900 & 0.82354100 & -2.13963200 \\
\hline O - 0.75031300 & 1.64563200 & -0.95903500 \\
\hline O -5.53101800 & 1.59077800 & 0.00689100 \\
\hline O 0.06564100 & 0.76882700 & 1.02190100 \\
\hline N -7.15044700 & -1.34840100 & -1.41765900 \\
\hline$N-3.05016600$ & 2.43898400 & 0.08918800 \\
\hline C -6.93492900 & -0.35963500 & -0.28446100 \\
\hline C - 2.18783400 & 1.58880000 & 1.00286900 \\
\hline C -6.38785100 & -1.00391400 & 0.99370600 \\
\hline C - 2.88255000 & 0.28980700 & 1.45147500 \\
\hline C -6.04190400 & 0.78590300 & -0.86441100 \\
\hline C -0.84310100 & 1.32322900 & 0.26727700 \\
\hline H -7.90779800 & 0.08400200 & -0.04524400 \\
\hline H -1.97097700 & 2.16081500 & 1.90955800 \\
\hline H -6.12292700 & -0.20917300 & 1.69091200 \\
\hline H -7.12968600 & -1.65788500 & 1.46528600 \\
\hline H -2.23894700 & -0.23655200 & 2.15608700 \\
\hline H -3.84452300 & 0.51746400 & 1.91350900 \\
\hline H -8.11493900 & -1.69087900 & -1.47461100 \\
\hline H -6.51175500 & -2.15309200 & -1.32345900 \\
\hline H -3.04603100 & 3.41748100 & 0.39702600 \\
\hline H -4.08033800 & 2.11177200 & 0.02307600 \\
\hline H -6.89018500 & -0.81182100 & -2.27829500 \\
\hline$H-2.60580000$ & 2.39887900 & -0.84779700 \\
\hline Au 2.04000600 & 0.23089800 & 0.31768900 \\
\hline Au 4.42363400 & -0.44793700 & -0.38337200 \\
\hline \multicolumn{3}{|c|}{$H=-937.205856$ Hartree } \\
\hline תר דבים - & & \\
\hline
\end{tabular}

Structure 4

$\begin{array}{ccc}\text { S 5.54794600 } & -2.17780700 & -0.43337300 \\ \text { S 4.45221800 } & -0.65838100 & -1.75529000 \\ \text { O } 4.74836100 & 1.29277200 & 1.23160100 \\ \text { O } 0.48334700 & 2.18056200 & 0.10299900 \\ \text { O } 6.67701300 & 1.82663100 & 0.06780600 \\ \text { O -0.00160000 } & 0.12484600 & -0.83903600 \\ \text { N } 5.69426400 & -0.74694400 & 2.46862300 \\ \text { N } 3.02235100 & 2.16548100 & -0.55750200\end{array}$

\begin{tabular}{lcc} 
C 6.64889200 & -0.18355900 & 1.42304700 \\
C 2.22942200 & 0.99620400 & -1.11035900 \\
C 7.00157500 & -1.19986300 & 0.32157200 \\
C 2.84004500 & -0.36812600 & -0.75480200 \\
C 5.99145100 & 1.10533700 & 0.84497000 \\
C 0.77700000 & 1.12890700 & -0.54749800 \\
H 7.57756500 & 0.10152900 & 1.92507900 \\
H 2.15594600 & 1.11059600 & -2.19718500 \\
H 7.53418600 & -0.68378900 & -0.47814300 \\
H 7.63774700 & -2.00197000 & 0.71192300 \\
H 3.08509200 & -0.42994700 & 0.30602400 \\
H 2.13566300 & -1.15199400 & -1.02796700 \\
H 4.89270900 & -0.06974600 & 2.46340900 \\
H 6.11743200 & -0.80090400 & 3.40097900 \\
H 3.53377800 & 2.66430100 & -1.29139400 \\
H 3.73040400 & 1.86588600 & 0.20496500 \\
H 5.33690100 & -1.67573200 & 2.20483100 \\
H 2.31881100 & 2.80047800 & -0.13029200 \\
Au -2.08293000 & 0.01233300 & -0.25330900 \\
Au -4.56596100 & -0.24716000 & 0.37963800 \\
H= -937.205970 Hartree & \\
G= -937.283234 Hartree & \\
\hline & & \\
\hline
\end{tabular}

Structure 5

$\begin{array}{lrr}\text { S 5.17285200 } & -0.25641100 & -2.10266200 \\ \text { S } 3.11828600 & 0.07971500 & -1.15254200 \\ \text { O } 4.44085000 & -1.97259800 & 1.80630900 \\ \text { O } 0.19680600 & 0.95788400 & 0.65857400 \\ \text { O } 4.85453000 & 0.30385200 & 1.74826600 \\ \text { O } 0.28135100 & 2.70252000 & -0.86135700 \\ \text { N } 6.13561900 & -2.59466500 & -0.05236900 \\ \text { N } 2.49359100 & 1.45749200 & 1.78562200 \\ \text { C } 6.27845600 & -1.17020900 & 0.45628800 \\ \text { C } 2.23274400 & 2.23223500 & 0.50635000 \\ \text { C } 6.41626600 & -0.13263500 & -0.66294100 \\ \text { C } 3.25170900 & 1.91039300 & -0.60333400 \\ \text { C } 5.07162300 & -0.92670900 & 1.42106200 \\ \text { C } 0.77162900 & 1.97347000 & 0.04175200 \\ \text { H } 7.18861300 & -1.12857100 & 1.06459400 \\ \text { H } 2.32901800 & 3.30008800 & 0.72307200 \\ \text { H } 6.35318800 & 0.86083300 & -0.21910800 \\ \text { H } 7.37425700 & -0.22876200 & -1.18583600 \\ \text { H } 3.05565000 & 2.54324200 & -1.46911300 \\ \text { H } 4.26770100 & 2.07043600 & -0.23872100 \\ \text { H } 7.02546200 & -3.10239300 & -0.08339900\end{array}$




$$
\begin{array}{lll}
\text { H } 5.71026600 & -2.60289300 & -0.99218000 \\
\text { H } 2.41445200 & 2.07443000 & 2.60144400 \\
\text { H } 3.46058100 & 0.97308400 & 1.81547500 \\
\text { H } 5.47028500 & -3.04119400 & 0.62156300 \\
\text { H } 1.74103400 & 0.74743000 & 1.84879100 \\
\text { Au -1.80294900 } & 0.26598100 & 0.22348500 \\
\text { Au }-4.16889900 & -0.65751100 & -0.20203500 \\
\text { H= -937.205566 Hartree } & \\
\text { G= -937.282018 Hartree } &
\end{array}
$$

\begin{tabular}{|c|c|c|}
\hline S -6.00670100 & -1.43668800 & 0.85831800 \\
\hline S -4.76293700 & -2.54836600 & -0.70628200 \\
\hline כ-5.47882600 & 3.58772300 & -0.50850700 \\
\hline O -0.12330500 & -0.31442300 & -0.46833900 \\
\hline O -4.13320100 & 1.85036600 & -1.20704800 \\
\hline O - 0.62841700 & -1.45943600 & 1.47830000 \\
\hline N -6.70266600 & 2.32461300 & 1.35242300 \\
\hline N -2.52517300 & -0.24213500 & -1.42548400 \\
\hline C -5.71964000 & 1.44108300 & 0.58974300 \\
\hline C - 2.41904900 & -0.93068000 & -0.08011900 \\
\hline C - 6.39002000 & 0.20712800 & -0.05156300 \\
\hline C - 2.97101500 & -2.36333700 & -0.08549600 \\
\hline C - 5.05015400 & 2.37936200 & -0.47892400 \\
\hline C -0.93093900 & -0.92188700 & 0.37945200 \\
\hline - -4.94117900 & 1.13518600 & 1.29189400 \\
\hline
\end{tabular}

Structure 6

$$
\begin{aligned}
& \text { S } 6.39133000 \quad 0.93636600-1.28902000 \\
& \text { S } 5.01598700 \quad 1.79537300 \quad 0.33289300 \\
& \begin{array}{llll}
0 & 3.95543300 & -1.65208700 & 0.43205400
\end{array} \\
& \begin{array}{llll}
0 & 0.01732100 & 0.40587500 & 0.73432900
\end{array} \\
& \begin{array}{llll}
0 & 5.66663000 & -1.56180700 & 1.98821500
\end{array} \\
& \begin{array}{llll}
0 & 0.48123300 & 2.13287000 & -0.73251100
\end{array} \\
& \text { N } 5.43077600 \quad-2.13738500-1.61399600 \\
& \text { N } 2.41363000 \quad 0.111860001 .64650900 \\
& \text { C } 6.23188100-1.84502700-0.35214000 \\
& \text { C } 2.24075300 \quad 1.33012500 \quad 0.75766200 \\
& \text { C } 7.15820400-0.62451600-0.50318100 \\
& \text { C } 3.27671600 \quad 1.40135200-0.37482600 \\
& \text { C } 5.21362900-1.67500400 \quad 0.81463900 \\
& \begin{array}{lll}
\text { C } 0.79303400 & 1.31035200 & 0.16892400
\end{array} \\
& \text { H } 6.85758900-2.71514200-0.13397900 \\
& \text { H } 2.30298000 \quad 2.22378600 \quad 1.38883900 \\
& \text { H 7.56632900 }-0.36564600 \quad 0.47440900 \\
& \text { H } 7.98488400-0.84014500-1.18911100 \\
& \text { H } 3.33471000 \quad 0.45740600-0.91702800 \\
& \text { H } 3.00321300 \quad 2.20870400-1.05263900 \\
& \text { H } 4.43683700-2.16312300-1.28070600 \\
& \text { H } 5.69254500-3.02546900-2.05427300 \\
& \text { H } 2.86017800 \quad 0.34713600 \quad 2.53803800 \\
& \text { H } 2.99874800 \quad-0.66607700 \quad 1.17459900 \\
& \text { H } 5.51899900-1.37863300-2.30479100 \\
& \text { H } 1.45317700 \quad-0.24328300 \quad 1.81637200 \\
& \text { Au -2.06231700 } 0.11544000 \quad 0.22059400 \\
& \mathrm{Au}-4.54386500 \quad-0.33507500-0.29442300 \\
& \mathrm{H}=-937.205495 \text { Hartree } \\
& \mathrm{G}=-937.281580 \text { Hartree }
\end{aligned}
$$

Structure 7

S $4.13501000 \quad-2.209355001 .08240700$ S $5.86803200-1.62405600-0.27782000$

\begin{tabular}{lcc} 
O -0.05674200 & -0.86052500 & -0.04188800 \\
O 5.64223900 & 3.09232000 & 0.66782000 \\
O 0.57280400 & 0.29713900 & -1.94216700 \\
O 4.40039000 & 1.49653400 & -0.42340200 \\
N 3.11282300 & -0.35287600 & -1.78351500 \\
N 7.91314800 & 1.98408600 & 0.46501100 \\
C 2.21751100 & -1.09000100 & -0.80345700 \\
C 6.78781400 & 1.10062600 & -0.08318000 \\
C 2.72977900 & -1.00453900 & 0.63898300 \\
C 6.75824400 & -0.24081900 & 0.68681400 \\
C 0.79014500 & -0.47497700 & -0.95631900 \\
C 5.49084300 & 1.96716800 & 0.06296600 \\
H 2.14815100 & -2.13616900 & -1.11929900 \\
H 6.98760900 & 0.92148900 & -1.14221800 \\
H 3.04186800 & 0.00817200 & 0.89813600 \\
H 1.92878600 & -1.32565400 & 1.31164200 \\
H 7.77656900 & -0.61659500 & 0.82252800 \\
H 6.27710700 & -0.14891300 & 1.66310600 \\
H 3.73965700 & -0.98771500 & -2.28567100 \\
H 3.70731600 & 0.38402300 & -1.28537300 \\
H 8.58550400 & 2.25274500 & -0.26146400 \\
H 8.42791100 & 1.55045800 & 1.23927800 \\
H 2.47241800 & 0.12183000 & -2.44882300 \\
H 7.38972500 & 2.83528600 & 0.80968600 \\
Au -2.12986400 & -0.24350100 & 0.00609500 \\
Au -4.61480200 & 0.41196500 & 0.18120700 \\
H= -937.203639 Hartree & \\
G= -937.280932 Hartree & \\
\hline & & \\
& &
\end{tabular}

Structure 8 


$\begin{array}{ccc}\text { H-2.97912400 } & -0.33270700 & 0.64590600 \\ \text { H-7.48004000 } & 0.28873100 & -0.07613400 \\ \text { H-6.03566200 } & 0.07329800 & -1.07437200 \\ \text { H-2.41401100 } & -3.01308700 & -0.77145900 \\ \text { H-2.90753100 } & -2.77686200 & 0.92069200 \\ \text { H-7.67143300 } & 1.99167800 & 1.30149900 \\ \text { H-6.61526000 } & 3.25390900 & 0.86082800 \\ \text { H-3.17533100 } & 0.60448400 & -1.36039700 \\ \text { H-2.88299800 } & -0.86973300 & -2.15419100 \\ \text { H-6.44926800 } & 2.42862500 & 2.34085100 \\ \text { H-1.56001600 } & 0.05673800 & -1.66553300 \\ \text { Au 1.99834600 } & -0.06779400 & -0.13155200 \\ \text { Au 4.53096800 } & 0.29766000 & 0.15465900 \\ \text { H= -937.203502 Hartree } & \\ \text { G=-937.280717 Hartree } & \end{array}$

Structure 9

$\begin{array}{lrr}\text { S } 2.86888700 & 0.12296900 & -1.50267300 \\ \text { S } 4.98856200 & -0.29755900 & -2.23358700 \\ \text { O } 0.06466000 & 2.79396300 & -0.67225400 \\ \text { O } 6.00450700 & -1.02448300 & 2.46283600 \\ \text { O } 0.14435000 & 0.84032500 & 0.56741600 \\ \text { O } 5.00480700 & 0.58813300 & 1.14623800 \\ \text { N } 2.52557200 & 1.27871100 & 1.57508500 \\ \text { N } 7.56646200 & -1.95572300 & 0.67219900 \\ \text { C } 2.14835400 & 2.15819100 & 0.39986900 \\ \text { C } 6.59755100 & -0.90581500 & 0.11763500 \\ \text { C } 3.05762200 & 1.90535400 & -0.81717900 \\ \text { C 5.69655500 } & -1.53282800 & -0.97166100 \\ \text { C } 0.64925400 & 1.94862500 & 0.05810600 \\ \text { C 5.79927000 } & -0.39878800 & 1.36010600 \\ \text { H } 2.28606600 & 3.20333700 & 0.69301400 \\ \text { H } 7.18591600 & -0.08871400 & -0.30403600 \\ \text { H } 2.78871500 & 2.59807000 & -1.61530000 \\ \text { H } 4.10683300 & 2.01820100 & -0.54360300 \\ \text { H } 6.28737300 & -2.19901200 & -1.61022200 \\ \text { H } 4.86616500 & -2.10188300 & -0.54751900 \\ \text { H } 1.88561700 & 0.46744400 & 1.56011900 \\ \text { H } 2.37732300 & 1.77603100 & 2.45978100 \\ \text { H } 8.54212300 & -1.64252200 & 0.62389400 \\ \text { H 7.49478500 } & -2.86523400 & 0.20292400 \\ \text { H } 3.55175700 & 0.96094000 & 1.51661200 \\ \text { H 7.27058400 } & -2.04269100 & 1.68047700 \\ \text { Au }-1.89090400 & 0.20127200 & 0.23359100 \\ \text { Au }-4.29194300 & -0.67194800 & -0.09412400 \\ & & \end{array}$

$H=-937.201883$ Hartree

$\mathrm{G}=-937.280831$ Hartree

Structure 10

\begin{tabular}{|c|c|c|}
\hline S 5.17511100 & 1.52886000 & 1.54926500 \\
\hline S 4.36581700 & - & -0.24148400 \\
\hline O 6.39002100 & -3.11896100 & -0.51256100 \\
\hline O 0.57585300 & -0.23743500 & -2.16080200 \\
\hline O 5.00736100 & -1.56273700 & -1.50477100 \\
\hline O 0.15749200 & 0.49380000 & -0.00497900 \\
\hline N 6.37479400 & -2.10400400 & 1.84267700 \\
\hline N 3.16368100 & 0.24792200 & -2.07683500 \\
\hline C 5.62326000 & -1.25014700 & 0.82604600 \\
\hline C 2.41498200 & $0.61041800-$ & -0.81187000 \\
\hline C 6.21334700 & 0.16924400 & 0.68407300 \\
\hline C 2.57043000 & 2.08453100 & -0.41124000 \\
\hline C 5.68066400 & -2.05108900 & -0.52461200 \\
\hline C 0.91645600 & 0.24966700 & -1.03754000 \\
\hline H 4.57975200 & -1.20322800 & 1.14327300 \\
\hline H 2.78770400 & -0.02530400 & -0.00333000 \\
\hline H 7.21939100 & 0.24651200 & 1.10579000 \\
\hline H 6.26388700 & 0.45535500 & -0.36726000 \\
\hline H 2.15668600 & 2.75968700 & -1.16991200 \\
\hline H 2.05450400 & 2.25574500 & 0.53298500 \\
\hline H 7.13031400 & -1.60034400 & 2.31915000 \\
\hline H 6.76913000 & -2.88436000 & 1.25352500 \\
\hline H 3.91160100 & -0.49024200 & -1.87024400 \\
\hline H 3.62526000 & 1.06194800 & -2.49809500 \\
\hline H 5.75026900 & -2.49863100 & 2.55433100 \\
\hline H 2.45150500 & -0.12633000 & -2.73453900 \\
\hline $\mathrm{Au}-1.9709980 \mathrm{C}$ & $0 \quad 0.10319400$ & $0 \quad 0.04079800$ \\
\hline $\mathrm{Au}-4.50602500$ & $0-0.30689100$ & $0 \quad 0.23217200$ \\
\hline \multicolumn{3}{|c|}{$H=-937.203892$ Hartree } \\
\hline \multicolumn{3}{|c|}{$G=-937.280550$ Hartree } \\
\hline
\end{tabular}

Structure 11

$\begin{array}{lcc}\text { S } 3.04284900 & -1.21624500 & 0.04770000 \\ \text { S } 4.98101000 & -2.24029300 & -0.58365900 \\ \text { O }-0.16797300 & 0.71308500 & -0.98136200 \\ \text { O } 7.19312200 & 1.79559000 & 1.01497300 \\ \text { O } 0.71964300 & 1.30411900 & 1.07321600 \\ \text { O } 5.43924300 & 1.17612000 & -0.35301500 \\ \text { N } 3.04345000 & 2.10901800 & 0.05318700 \\ \text { N } 8.50126700 & -0.38621000 & 0.85223600 \\ \text { C } 2.14196400 & 1.34957000 & -0.89788200\end{array}$




\begin{tabular}{ccc} 
C 7.17405600 & -0.44196600 & 0.08733000 \\
C 2.78800800 & 0.03833200 & -1.38216200 \\
C 6.29133800 & -1.58733100 & 0.63208200 \\
C 0.78417400 & 1.11539800 & -0.18216700 \\
C 6.54647300 & 0.97633400 & 0.26627400 \\
H 1.96702000 & 1.96983300 & -1.78168000 \\
H 7.40099500 & -0.61043200 & -0.96750500 \\
H 2.13405000 & -0.43006200 & -2.11794100 \\
H 3.77460700 & 0.22744600 & -1.80542700 \\
H 6.90334000 & -2.47871600 & 0.81105200 \\
H 5.78688700 & -1.31631200 & 1.56235000 \\
H 2.70023400 & 1.91906600 & 1.01074100 \\
H 2.97043900 & 3.11919800 & -0.10766600 \\
H 9.31031400 & -0.52685300 & 0.23789500 \\
H 8.55346700 & -1.06624200 & 1.61884500 \\
H 4.07309800 & 1.81243200 & -0.05297800 \\
H 8.50592900 & 0.59247100 & 1.24588000 \\
Au -2.16503900 & 0.23754300 & -0.30072200 \\
Au -4.58310700 & -0.34615300 & 0.36923500 \\
H= -937.202097 Hartree & \\
G= -937.279864 Hartree & \\
\hline & &
\end{tabular}

Structure 12

\begin{tabular}{|c|c|c|}
\hline S -5.13509800 & -2.26511700 & -0.55597200 \\
\hline S -2.94154700 & -1.61551400 & -0.38859400 \\
\hline O -5.49155100 & 1.80511500 & 0.05073700 \\
\hline O -0.27581800 & 0.21485400 & -0.29232800 \\
\hline O -4.68855400 & 0.87770100 & 2.01056300 \\
\hline O - 0.43775700 & 2.52295200 & -0.19251400 \\
\hline N -7.33216100 & 0.07608600 & -0.38625100 \\
\hline $\mathrm{N}-3.08155300$ & 2.51061200 & -0.77217500 \\
\hline C - 6.57607500 & -0.17748700 & 0.91050300 \\
\hline C - 2.45528400 & 1.18706500 & -0.36872300 \\
\hline C -6.01162000 & -1.60817100 & 1.00824100 \\
\hline C - 2.91924600 & 0.05797800 & -1.30663500 \\
\hline C -5.48394000 & 0.92645700 & 1.02519500 \\
\hline C -0.92304500 & 1.35483200 & -0.29084700 \\
\hline H -7.27799200 & -0.03878000 & 1.73893400 \\
\hline H - 2.81761500 & 0.99904700 & 0.64917600 \\
\hline H -5.31964300 & -1.66066000 & 1.84960600 \\
\hline H -6.81566800 & -2.33574800 & 1.15854800 \\
\hline H -3.93640600 & 0.22928700 & -1.66494900 \\
\hline H - 2.25008300 & -0.06483900 & -2.15985100 \\
\hline H -7.00320100 & 1.02313000 & -0.69106900 \\
\hline$H-8.35019600$ & 0.05377900 & -0.26818500 \\
\hline
\end{tabular}

H -3.04463300 $2.66183400 \quad-1.78675300$

H - $2.54509700 \quad 3.26853500-0.32515600$

H -7.05707500 $-0.60101600-1.11332300$

$\begin{array}{lll}H-4.09980300 & 2.47790000 & -0.44168300\end{array}$

$\begin{array}{lll}\text { Au } 1.86748600 & 0.06325000 & -0.07644700\end{array}$

$\begin{array}{lll}\text { Au } 4.41105100 & -0.26373500 & 0.17516400\end{array}$

$\mathrm{H}=-937.203400$ Hartree

$\mathrm{G}=-937.279756$ Hartree

Structure 13

S -4.15933500 $0.87219500 \quad 1.66075300$

S - $2.56052100-0.80215700 \quad 1.77347500$

O $-1.56142100 \quad 0.35698900 \quad-1.45823900$

$\begin{array}{llll}0 & -4.68754100 & -0.45766700 & -1.62485300\end{array}$

$\begin{array}{llll}0 & -0.39872000 & 1.73709700 & -0.02875000\end{array}$

$\begin{array}{llll}0 & -6.12499900 & -1.71421500 & -0.32777100\end{array}$

N-3.76052200 $1.96297100-1.46582600$

N-2.60699300 $-2.11824900-1.29113000$

$\begin{array}{llll}C-2.66978600 & 2.30171600 & -0.47536000\end{array}$

$\begin{array}{lll}\text { C }-3.81529000 & -2.37481200 & -0.41002700\end{array}$

C $-3.16451400 \quad 2.34355500 \quad 0.98373100$

$\begin{array}{lll}\text { C }-3.48561000 & -2.32034100 & 1.09100300\end{array}$

C - $1.45717400 \quad 1.36477000-0.68356300$

$\begin{array}{llll}\text { C }-4.98659700 & -1.43170900 & -0.81460400\end{array}$

H - $2.32434800 \quad 3.31591900-0.70684200$

$\begin{array}{lll}H-4.14464200 & -3.40007300 & -0.61374900\end{array}$

H - $2.30323000 \quad 2.51208600 \quad 1.63185300$

H -3.86950300 $3.17069500 \quad 1.12715000$

H -4.41644500 $-2.43854000 \quad 1.64829300$

H - $2.80717000 \quad-3.13301200 \quad 1.37695000$

H -3.41465500 $2.11503000 \quad-2.42115300$

H $-4.57524500 \quad 2.57195600-1.32577900$

H-1.81506000 $-2.71408000-1.02125400$

H -2.29190600 $-1.10704200-1.28021100$

$\mathrm{H}-4.09669900 \quad 0.93033300-1.42805600$

$\begin{array}{lll}H-2.84927300 & -2.33083300 & -2.26620100\end{array}$

Au $1.46649900 \quad 0.62193000 \quad-0.04055700$

$\begin{array}{lll}\text { Au } 3.73165000 & -0.59918100 & 0.02789700\end{array}$

$\mathrm{H}=-937.204798$ Hartree

$\mathrm{G}=-937.279521$ Hartree

Structure 14

S -5.79209300 $-1.59218500-0.80947100$

S - $4.08554100 \quad-2.27289100 \quad 0.58851300$

$\begin{array}{llll}0-7.13546500 & 2.70064800 & 0.00361700\end{array}$ 


\begin{tabular}{|c|c|c|}
\hline O -0.53767800 & 1.01200300 & -1.63508100 \\
\hline & 300 & 800 \\
\hline 0.02172400 & -0.44631600 & 0.07058700 \\
\hline$N-7.23934900$ & 1.19348400 & 2.06624800 \\
\hline N -3.10645000 & 0.43827700 & -1.60368400 \\
\hline C -6.29512600 & 0.66300500 & 0.98478600 \\
\hline C -2.31182500 & -0.18687300 & -0.47662200 \\
\hline C -6.88257300 & -0.64834100 & 0.41988300 \\
\hline C -2.52968200 & -1.69832000 & -0.34478500 \\
\hline C -6.20208300 & 1.81943800 & -0.06406000 \\
\hline C -0.80909300 & 0.16402700 & -0.72867200 \\
\hline$H-5.32308200$ & 0.47350900 & 1.44318600 \\
\hline$H-2.59636600$ & 0.31733000 & 0.45240900 \\
\hline H -7.10274700 & -1.34220800 & 1.23575200 \\
\hline$H-7.80388400$ & -0.46726000 & -0.14619000 \\
\hline$H-2.50118900$ & -2.20252900 & -1.31484800 \\
\hline H -1.72901400 & -2.10692700 & 0.27934600 \\
\hline H -7.73373400 & 1.98724600 & 1.58421800 \\
\hline H -6.72092600 & 1.56399000 & 2.87108200 \\
\hline H - 2.45809200 & 1.10555900 & -2.06636100 \\
\hline H -3.98870800 & 0.95240600 & -1.25888200 \\
\hline H -7.90906200 & 0.49512400 & 2.40743100 \\
\hline H -3.39307100 & -0.26006800 & -2.29785300 \\
\hline Au 2.15931200 & -0.10457500 & 0.06277700 \\
\hline Au 4.71279500 & 0.21520900 & 0.16520000 \\
\hline \multicolumn{3}{|c|}{$H=-937.202504$ Hartree } \\
\hline \multicolumn{3}{|c|}{$G=-937.279770$ Hartree } \\
\hline
\end{tabular}

Structure 15

\begin{tabular}{|c|c|c|}
\hline 0 & & 1. \\
\hline S -4.97421100 & -0.59337800 & 2.17920200 \\
\hline O -0.13553700 & 0.87900200 & -0.45781000 \\
\hline O -4.99386400 & 0.65160300 & -1.06529500 \\
\hline O -0.06539700 & 2.68691300 & 0.98689700 \\
\hline O -6.35752500 & -0.45797800 & -2.56177300 \\
\hline N -2.51982900 & 1.40724600 & -1.40884600 \\
\hline N -7.60068000 & -1.88421200 & -0.85120800 \\
\hline C - 2.14601200 & 2.16040300 & -0.14784000 \\
\hline C -6.62100300 & -0.90532400 & -0.19633100 \\
\hline C - 3.05354900 & 1.77872000 & 1.03623700 \\
\hline C -5.65614300 & -1.65157700 & 0.74998100 \\
\hline C - 0.64581600 & 1.92258000 & 0.16915900 \\
\hline C - 5.92352600 & -0.17184100 & -1.38705800 \\
\hline H -2.28811600 & 3.22954100 & -0.33058800 \\
\hline H -7.21339300 & -0.17921700 & 0.36610000 \\
\hline
\end{tabular}

$\begin{array}{lll}H-2.78904100 & 2.38878800 & 1.90059100\end{array}$

H $-4.10346500 \quad 1.91310200 \quad 0.77562300$

$\begin{array}{lll}\mathrm{H}-6.18967700 & -2.44866500 & 1.27900900\end{array}$

$\begin{array}{lll}H-4.80733700 & -2.08758000 & 0.21903200\end{array}$

H - $1.87802600 \quad 0.60010400-1.47717500$

H - 2.37079200 $1.99388600-2.23702700$

H -7.55486700 $-1.61812400-1.87003200$

$\begin{array}{lll}H-8.55504800 & -1.78996500 & -0.48905400\end{array}$

H -3.54125900 $1.08364700 \quad-1.38623400$

H -7.30877500 $-2.86325200-0.75054000$

Au $1.90519400 \quad 0.21971500 \quad-0.20600800$

$\begin{array}{lll}\text { Au } 4.31474000 & -0.66618100 & 0.00087500\end{array}$ $\mathrm{H}=-937.201768$ Hartree

$\mathrm{G}=-937.279544$ Hartree

\section{Structure 16}

\begin{tabular}{|c|c|c|}
\hline 01600 & -1.22691200 & 0.05867000 \\
\hline & -2.24 & -0.57120100 \\
\hline O 0.70912000 & 1.27461700 & 1.14681600 \\
\hline O 5.44462800 & 1.16670100 & -0.27018000 \\
\hline O -0.16916800 & 0.76472800 & -0.93353200 \\
\hline O 7.39720600 & 1.82334600 & 0.77199900 \\
\hline N 3.04879800 & 2.09048000 & 0.17101100 \\
\hline N 8.52678300 & -0.46449900 & 0.78542000 \\
\hline C 2.14565300 & 1.37433800 & -0.81196200 \\
\hline C 7.17691200 & -0.48090400 & 0.05976000 \\
\hline C 2.78084600 & 0.07248300 & -1.33412200 \\
\hline C 6.26030700 & -1.57784800 & 0.64209600 \\
\hline C 0.78061500 & 1.12993900 & -0.11392900 \\
\hline C 6.62085100 & 0.97212800 & 0.20425500 \\
\hline H 1.98425400 & 2.02609700 & -1.67528200 \\
\hline H 7.37781400 & -0.67877600 & -0.99622400 \\
\hline H 2.12677900 & -0.36528000 & -2.08846300 \\
\hline H 3.77213700 & 0.26421800 & -1.74507400 \\
\hline H 6.84576700 & -2.47493800 & 0.87120000 \\
\hline H 5.74607900 & -1.25173600 & 1.54868100 \\
\hline H 2.70600500 & 1.85961800 & 1.11953200 \\
\hline H 2.97646100 & 3.10688700 & 0.05478300 \\
\hline H 8.66112500 & 0.55625200 & 1.01113400 \\
\hline H 9.29242300 & -0.81422900 & 0.20001900 \\
\hline H 4.07443400 & 1.79843100 & 0.05106100 \\
\hline H 8.51261900 & -1.00002900 & 1.66117500 \\
\hline $\mathrm{Au}-2.1701840 \mathrm{C}$ & $0 \quad 0.25990700$ & $0-0.28641300$ \\
\hline $\mathrm{Au}-4.59143300$ & $0 \quad-0.36200100$ & $0 \quad 0.33572300$ \\
\hline
\end{tabular}


$G=-937.279338$ Hartree

Structure 17

\begin{tabular}{|c|c|c|}
\hline 0 & & \\
\hline & & \\
\hline 93000 & 00 & \\
\hline 400 & 0 & -1. \\
\hline-6.12494200 & -1.71416900 & -0.3 \\
\hline 00 & 1.7 & -0 \\
\hline 500 & -2.1 & -1 \\
\hline-3.76266100 & 1.96529700 & -1.46 \\
\hline 00 & -2.373 & -0. \\
\hline 100 & & \\
\hline-3.48256300 & -2.32248600 & 1.08 \\
\hline 4100 & 2.34 & 0.9 \\
\hline 0 & & \\
\hline 300 & 700 & -0.6 \\
\hline 3600 & -3.397 & -0.6 \\
\hline-2 . & 3.3 & -0.7 \\
\hline 00 & -2.4 & \\
\hline-2.80391100 & -3.13617000 & 1.36 \\
\hline-2.30098400 & 2.51067000 & 1.63 \\
\hline 500 & 00 & 900 \\
\hline-2.85229300 & -2.32621700 & -2.27 \\
\hline-1.81556000 & -2.71002900 & -1.02879900 \\
\hline-4.57707500 & 2.57426800 & -1.3 \\
\hline-4.09904300 & 0.93270200 & -1.42 \\
\hline-2.29387900 & -1.10314300 & -1.28503100 \\
\hline-3.41790100 & 2.11841900 & -2.41731300 \\
\hline u 1.46627500 & 0.62271700 & $-0.0409260 c$ \\
\hline 3.73065100 & -0.59975000 & 0.027748 \\
\hline \multicolumn{3}{|c|}{$H=-937.204794$ Hartree } \\
\hline \multicolumn{3}{|c|}{ נואר } \\
\hline
\end{tabular}

Structure 18

$\begin{array}{crc}\text { S }-6.31085400 & -1.75511000 & -0.09387200 \\ \text { S }-4.04488200 & -2.08716400 & 0.03958300 \\ \text { O }-4.86713500 & 2.08706000 & -0.42775200 \\ \text { O }-0.78259600 & -1.60384600 & -0.15886400 \\ \text { O }-4.44253500 & 1.37903500 & 1.73172700 \\ \text { O }-0.04263400 & 0.57861300 & -0.38815000 \\ \text { N }-7.29516500 & 1.27907800 & -0.55351500 \\ \text { N }-2.39541900 & 1.51573500 & -1.19506900 \\ \text { C -6.65276400 } & 1.01442900 & 0.80108600 \\ \text { C }-2.40005300 & 0.17087300 & -0.48820500\end{array}$

$$
\begin{array}{ccc}
\text { C -6.74681100 } & -0.45998500 & 1.24011400 \\
\text { C-3.34717400 } & -0.81216600 & -1.19805300 \\
\text { C -5.18892200 } & 1.53804400 & 0.71958200 \\
\text { C-0.96048600 } & -0.36651400 & -0.34415700 \\
\text { H-7.18256400 } & 1.61787500 & 1.54504400 \\
\text { H-2.77162500 } & 0.38427700 & 0.52153000 \\
\text { H-6.10287100 } & -0.61427700 & 2.10683400 \\
\text { H-7.77545500 } & -0.72357800 & 1.50605600 \\
\text { H-4.20582200 } & -0.29482700 & -1.63069200 \\
\text { H-2.83532400 } & -1.38206800 & -1.97541500 \\
\text { H-6.60002200 } & 1.88819700 & -1.04815900 \\
\text { H- } 8.20823900 & 1.73891100 & -0.48017500 \\
\text { H-2.29906000 } & 1.41708400 & -2.21235400 \\
\text { H-1.58219000 } & 2.04815100 & -0.85362700 \\
\text { H- -7.39491700 } & 0.40874600 & -1.09654900 \\
\text { H -3.32481100 } & 1.98784300 & -0.95369000 \\
\text { Au 2.06435000 } & 0.20141700 & -0.11368800 \\
\text { Au 4.60229800 } & -0.10772900 & 0.20535900 \\
\text { H= -937.203596 Hartree } & \\
\text { G=-937.279363 Hartree } &
\end{array}
$$

\begin{tabular}{|c|c|c|}
\hline & & \\
\hline 60100 & 1.814 & -1 \\
\hline-3.82878500 & -3.99576200 & $1.28 \mathrm{~s}$ \\
\hline-0.30249500 & 1.91584200 & 0. \\
\hline 500 & 100 & \\
\hline 800 & 0.42208700 & 1. \\
\hline-2.28845500 & -2.03816300 & 0.616 \\
\hline 900 & 300 & 0.1397310 \\
\hline 0 & -1.8 & \\
\hline-2.50074700 & 2.64518200 & 1.072 \\
\hline-3.86623600 & -1.44744600 & -1.2 \\
\hline 0 & & \\
\hline 700 & 900 & 0.5 \\
\hline C - 1.40980900 & 1.53998200 & 1.0161330 \\
\hline-4.15784200 & -1.07936300 & 0.82 \\
\hline 00 & 900 & 2.4 \\
\hline H -3.42597100 & -2.18951400 & -1.96825 \\
\hline H -4.92129300 & -1.33476900 & -1.5396 \\
\hline-4.08386200 & 1.22438600 & \\
\hline -4.67131700 & 2.87568000 & 1.081 \\
\hline H -2.34709300 & -2.85026000 & 1.27544 \\
\hline-1.91224500 & -1.16888900 & 1.06 \\
\hline-1.6743990 & -2.30361800 & -0.16 \\
\hline
\end{tabular}

Structure 19 


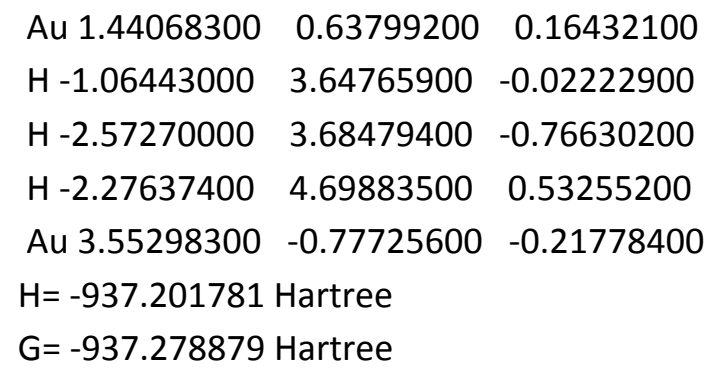

Structure 2

\begin{tabular}{lrr} 
S -6.56470100 & 1.04122400 & -2.05414600 \\
S -5.90385200 & -1.08752100 & -1.51243500 \\
O -5.91192600 & 0.34875300 & 1.76206200 \\
O -1.99221300 & -1.88932000 & 1.84740200 \\
O -8.05618700 & -0.49487900 & 1.53695100 \\
O -1.47373100 & -1.75212100 & -0.40100800 \\
N -6.38597800 & 2.65978500 & 0.75650100 \\
N-4.59598700 & -1.91024200 & 1.50464700 \\
C-7.56205100 & 1.69961700 & 0.63190000 \\
C-3.79823700 & -1.91912600 & 0.21438900 \\
C-7.97197900 & 1.43820800 & -0.82844800 \\
C-4.17441300 & -0.77473500 & -0.74097900 \\
C-7.16981600 & 0.39022500 & 1.37966200 \\
C-2.29018000 & -1.84090100 & 0.61423800 \\
H-8.42025100 & 2.14226500 & 1.14487100 \\
H-3.94409000 & -2.88752800 & -0.27605000 \\
H-8.70131600 & 0.62756500 & -0.85144000 \\
H-8.41880600 & 2.33190700 & -1.27830900 \\
H-4.20014300 & 0.18574000 & -0.22519900 \\
H-3.45206800 & -0.74544400 & -1.55510500 \\
H-5.26681000 & -2.68325400 & 1.54342100 \\
H-5.13996100 & -0.98495300 & 1.66201300 \\
H -3.89768100 & -2.01961400 & 2.26659300 \\
H -5.96587900 & 2.87627600 & -0.15798000 \\
H -6.64040800 & 3.53449200 & 1.22733700 \\
H -5.68502400 & 2.12267000 & 1.32310400 \\
Au 2.29865200 & 3.25642400 & 0.07453900 \\
Au 2.40984700 & 0.64692500 & -0.00362300 \\
Au 0.66675900 & -1.61913500 & -0.16364800 \\
Au 3.27506600 & -1.93820600 & -0.02339300 \\
H=-1208.197279 Hartree & \\
G=-1208.293239 Hartree & \\
& & \\
\hline
\end{tabular}

Structure 3

$\begin{array}{lrc}\text { S } 6.11186200 & 0.13131200 & 1.82414800 \\ \text { S } 7.06441700 & -1.54194400 & 0.58974500 \\ \text { O } 1.58758100 & 1.42702100 & -0.46339100 \\ \text { O } 8.37473000 & 2.50170000 & -0.19085100 \\ \text { O } 1.76211800 & -0.76350200 & 0.25765600 \\ \text { O } 6.39616500 & 1.92242000 & -1.24275800 \\ \text { N } 4.12365500 & 2.02487100 & 0.03501900 \\ \text { N } 9.54827900 & 0.22069800 & -0.54134300 \\ \text { C } 3.76940200 & 0.55875700 & 0.16704600 \\ \text { C } 8.24425500 & 0.36185700 & -1.30885000\end{array}$




$\begin{array}{lll}\text { C } 4.24663500 & -0.05548700 & 1.48916600 \\ \text { C } 7.31905200 & -0.85330800 & -1.17269400 \\ \text { C } 2.23087400 & 0.42340900 & -0.02811400 \\ \text { C 7.60952400 } & 1.72023800 & -0.85737500 \\ \text { H } 4.24189900 & 0.04404500 & -0.67535700 \\ \text { H } 8.49607300 & 0.46934900 & -2.36968400 \\ \text { H 3.78361000 } & 0.43122100 & 2.35570200 \\ \text { H 3.99565800 } & -1.11535100 & 1.51023700 \\ \text { H 6.35505400 } & -0.60315500 & -1.61293600 \\ \text { H 7.73096300 } & -1.72734000 & -1.68987600 \\ \text { H 3.34559900 } & 2.47065700 & -0.48341200 \\ \text { H 5.05731300 } & 2.13025900 & -0.48507600 \\ \text { H 10.32804700 } & -0.09620900 & -1.12664700 \\ \text { H 9.43584500 } & -0.42837200 & 0.25266800 \\ \text { H 4.19433900 } & 2.48599500 & 0.94906500 \\ \text { H 9.72587200 } & 1.18325700 & -0.16683100 \\ \text { Au -0.32186100 } & -1.27170000 & 0.03961100 \\ \text { Au -2.73161900 } & 0.28009700 & -0.02142500 \\ \text { Au -2.66847500 } & -2.44188200 & -0.18345100 \\ \text { Au -3.50206300 } & 2.77491400 & 0.06202200 \\ \text { H= -1208.199288 Hartree } & \\ \text { G= -1208.292707 Hartree } & \end{array}$

Structure 4

\begin{tabular}{|c|c|c|}
\hline S -6.68273900 & 1.23384000 & -0.72870300 \\
\hline S -4.48623900 & 0.82096100 & -1.21978300 \\
\hline O -4.02914700 & 0.60086200 & 2.64369200 \\
\hline 1.49803000- 0 & -2.13893200 & -0.81084900 \\
\hline O -4.67883100 & -1.33123000 & 1.55600600 \\
\hline O - 1.75441100 & -0.31694000 & -2.21325200 \\
\hline N -6.29665600 & 1.82286400 & 2.42273200 \\
\hline$N-4.07123000$ & -2.62354000 & -0.64980300 \\
\hline C -6.35608400 & 0.35267700 & 2.04249900 \\
\hline C -3.62294000 & -1.85029600 & -1.87373900 \\
\hline C -7.11209300 & 0.09045400 & 0.73451100 \\
\hline C -4.59004600 & -0.74658300 & -2.31445600 \\
\hline C -4.87812300 & -0.16778200 & 2.06708900 \\
\hline C - 2.17198400 & -1.35286700 & -1.62776400 \\
\hline H -6.88524800 & -0.17228600 & 2.84592500 \\
\hline H -3.56664500 & -2.57836800 & -2.69400700 \\
\hline H -6.95394300 & -0.94749700 & 0.44143500 \\
\hline H -8.18704700 & 0.26640500 & 0.85227000 \\
\hline H -4.32474600 & -0.42852500 & -3.32323600 \\
\hline$H-5.62606500$ & -1.09252200 & -2.31072300 \\
\hline$H-7.02522600$ & 2.09824100 & 3.08880100 \\
\hline
\end{tabular}

\author{
$\begin{array}{lll}H-6.36507400 & 2.41782800 & 1.58224700\end{array}$ \\ $\begin{array}{lll}H-4.86363800 & -3.23738100 & -0.86592100\end{array}$ \\ H $-4.33161500 \quad-2.00867100 \quad 0.18965200$ \\ $\begin{array}{lll}H-5.34186500 & 1.93472600 & 2.84054300\end{array}$ \\ H -3.26166100 $-3.19112100 \quad-0.35348600$ \\ Au $1.78273200 \quad 3.24893300 \quad-0.22710500$ \\ Au $0.54057000-1.74705100-0.23893500$ \\ Au $2.05282100 \quad 0.66531600 \quad 0.05741400$ \\ Au $3.04264400-1.82285100 \quad 0.57189400$ \\ $\mathrm{H}=-1208.196813$ Hartree \\ $\mathrm{G}=-1208.291848$ Hartree
}

\section{Structure 5}

\begin{tabular}{|c|c|c|}
\hline 56.1927 & -1 . & \\
\hline S 7.58335200 & -0.27532700 & -1.82277900 \\
\hline O 1.71606800 & 0.41457500 & 0.26951700 \\
\hline O 8.00159600 & -1.46298100 & 2.30589100 \\
\hline O 2.23743600 & 0.53986400 & -1.97954000 \\
\hline O 6.25486200 & 0.00217300 & 1.90814200 \\
\hline N 3.92200100 & -0.76638400 & 1.01122200 \\
\hline N 9.67268400 & -0.34658200 & 0.67164500 \\
\hline C 3.93335300 & -0.22705800 & -0.40451800 \\
\hline C 8.40578200 & 0.46107200 & 0.89860000 \\
\hline C 4.45334500 & -1.24345700 & -1.42941800 \\
\hline C 7.77272600 & 0.98085800 & -0.39748900 \\
\hline C 2.50866700 & 0.27662800 & -0.77793900 \\
\hline C 7.46254100 & -0.42818900 & 1.77722600 \\
\hline H 4.58141900 & 0.65533700 & -0.39280400 \\
\hline H 8.67544800 & 1.33094100 & 1.50780800 \\
\hline H 3.82811800 & -2.14333500 & -1.46373000 \\
\hline H 4.46066900 & -0.78941500 & -2.41999500 \\
\hline H 6.80234900 & 1.41247900 & -0.15749100 \\
\hline H 8.39760300 & 1.74963500 & -0.86616300 \\
\hline H 3.12080100 & -0.31595200 & 1.48676600 \\
\hline H 4.84850600 & -0.53199600 & 1.49804400 \\
\hline H 10.52899300 & 0.20359200 & 0.79446700 \\
\hline H 9.67486700 & -0.76680700 & -0.27027400 \\
\hline H 3.77093300 & -1.78112000 & 1.03566600 \\
\hline H 9.61374900 & -1.11402300 & 1.38259000 \\
\hline $\mathrm{Au}-0.30611000$ & $0 \quad 1.13660200$ & $0 \quad 0.09191700$ \\
\hline $\mathrm{Au}-2.80523300$ & $0 \quad-0.26273700$ & $0 \quad 0.04046600$ \\
\hline $\mathrm{Au}-2.58062800$ & $0 \quad 2.45615000$ & $0-0.00641300$ \\
\hline $\mathrm{Au}-3.76096700$ & $0 \quad-2.69438400$ & $\begin{array}{ll}0 & 0.05467200\end{array}$ \\
\hline \multicolumn{3}{|c|}{$H=-1208.198650$ Hartree } \\
\hline \multicolumn{3}{|c|}{$\bar{j}=-1208.291741$ Hartree } \\
\hline
\end{tabular}


Structure 6

\begin{tabular}{|c|c|c|}
\hline & 300 & \\
\hline 4.13610100 & 0.37102500 & 0.37392000 \\
\hline O 6.93402400 & 0.34136700 & -2.25637600 \\
\hline O 2.02620400 & -1.84413300 & -1.57149600 \\
\hline O 6.81664100 & -1.37046100 & -0.70270100 \\
\hline O 1.28065200 & -2.18667000 & 0.59230100 \\
\hline N 7.84060200 & 2.12814000 & -0.60767500 \\
\hline N 4.52295200 & -2.58883700 & -1.04974800 \\
\hline C 7.86359900 & 0.71304000 & -0.05574000 \\
\hline C 3.62608600 & -2.43579300 & 0.16389100 \\
\hline C 7.31353900 & 0.60621300 & 1.37017200 \\
\hline C 4.13671300 & -1.37670000 & 1.15912500 \\
\hline C 7.12656800 & -0.18241200 & -1.10444200 \\
\hline C 2.19018800 & -2.12824200 & -0.34475200 \\
\hline H 8.90905900 & 0.38602000 & -0.03561700 \\
\hline H 3.60090500 & -3.38854000 & 0.70002200 \\
\hline H 7.23668300 & -0.44948900 & 1.62989900 \\
\hline H 7.96981400 & 1.10300300 & 2.09346300 \\
\hline H 3.48529500 & -1.35992700 & 2.03265200 \\
\hline H 5.16243200 & -1.60381100 & 1.45408200 \\
\hline H 8.73060200 & 2.62195800 & -0.48340400 \\
\hline H 7.08633200 & 2.67780900 & -0.16816400 \\
\hline H 4.69432000 & -3.58088600 & -1.24673800 \\
\hline H 5.48224000 & -2.09307600 & -0.95164100 \\
\hline H 7.61689800 & 2.00582400 & -1.62259200 \\
\hline H 4.00121500 & -2.19176900 & -1.85313500 \\
\hline $\mathrm{Au}-0.79721500$ & $0 \quad-1.73321600$ & $0 \quad 0.23639700$ \\
\hline $\mathrm{Au}-2.21062700$ & $0 \quad 0.74304400$ & $0-0.04505800$ \\
\hline $\mathrm{Au}-3.41372500$ & $0-1.70462100$ & $0 \quad-0.04290800$ \\
\hline $\mathrm{Au}-1.76807900$ & $0 \quad 3.31661300$ & $0-0.13448900$ \\
\hline \multicolumn{3}{|c|}{$H=-1208.197152$ Hartree } \\
\hline \multicolumn{3}{|c|}{$G=-1208.290640$ Hartree } \\
\hline
\end{tabular}

Structure 7

$\begin{array}{lrl}\text { S-5.94628600 } & 1.20926600 & 1.15547000 \\ \text { S-3.70949200 } & 1.65938400 & 1.38112200 \\ \text { O-3.37437300 } & -1.97327500 & -0.27531800 \\ \text { O }-0.07506400 & 2.57509200 & -0.78407400 \\ \text { O-3.46843200 } & -0.07607700 & -1.58872300 \\ \text { O - } 0.86560400 & 2.91104500 & 1.36555700 \\ \text { N-5.86335600 } & -1.93775900 & 0.44273100 \\ \text { N }-2.52778700 & 2.45195500 & -1.78877600 \\ \text { C-5.53204300 } & -1.00488400 & -0.71064900\end{array}$
C - $2.33816500 \quad 3.30730300 \quad-0.54556700$
C $-6.15792500 \quad 0.38776100-0.55114100$
$\begin{array}{llll}C-3.53065800 & 3.30368500 & 0.41467000\end{array}$
$\begin{array}{lll}C-3.98356800 & -0.99167700 & -0.87401700\end{array}$
C - $0.98971400 \quad 2.89322600 \quad 0.11096000$
H -5.94005400 $-1.45742700-1.62158300$
H - $2.20908200 \quad 4.33687800 \quad-0.90381500$
H -5.75884000 $1.04398400-1.32435500$
H -7.24782500 $0.34393900-0.65178400$
H-3.37976500 $4.08343400 \quad 1.16184700$
$\begin{array}{llll}H-4.47124700 & 3.48834700 & -0.10936500\end{array}$
H-3.15457000 $2.90549300-2.46276100$
H - $2.89854200 \quad 1.47927400 \quad-1.59807100$
H - $1.58820400 \quad 2.35047500 \quad-2.20845500$
H -5.96966600 $-1.39809400 \quad 1.31714600$
$\begin{array}{lll}H-6.71835600 & -2.48114200 & 0.28143000\end{array}$
$\begin{array}{lll}H-5.03997800 & -2.56621800 & 0.54215900\end{array}$
$\mathrm{Au}-1.10836500 \quad-1.97044700 \quad-0.07847500$
Au $1.39012300 \quad-1.33274700 \quad 0.13207400$
Au $1.74519200 \quad 1.52013300 \quad-0.27147300$
$\begin{array}{lll}\text { Au } 3.85997100 & 0.05529600 & 0.21292900\end{array}$
$\mathrm{H}=-1208.202274$ Hartree
$G=-1208.290575$ Hartree

Structure 8

$\begin{array}{lrr}\text { S } 7.39319800 & -0.95536200 & -1.50299100 \\ \text { S } 6.45869900 & 1.12934300 & -1.31827200 \\ \text { O } 7.03928400 & 0.53333200 & 2.39895200 \\ \text { O } 1.50377400 & 1.79318300 & 0.12635600 \\ \text { O } 5.07327900 & -0.41197800 & 1.62501800 \\ \text { O } 1.86927700 & 1.42531000 & -2.12625100 \\ \text { N } 6.08056800 & -2.66883500 & 0.92308400 \\ \text { N } 3.98679900 & 1.86004400 & 0.84303400 \\ \text { C } 7.12962600 & -1.64769900 & 1.34387600 \\ \text { C } 3.77984500 & 1.82568200 & -0.66017500 \\ \text { C } 8.12887200 & -1.31462400 & 0.22077200 \\ \text { C } 4.58631400 & 0.71522700 & -1.35116700 \\ \text { C } 6.36911800 & -0.38576600 & 1.85025800 \\ \text { C } 2.25583000 & 1.65009100 & -0.94982100 \\ \text { H } 7.69665900 & -2.06448400 & 2.18084000 \\ \text { H } 4.05702000 & 2.80584000 & -1.06440500 \\ \text { H } 8.73745800 & -0.46404100 & 0.52991800 \\ \text { H } 8.78726600 & -2.16565400 & 0.01395900 \\ \text { H } 4.44385600 & -0.24517700 & -0.85546200 \\ \text { H } 4.27804700 & 0.65131400 & -2.39368300\end{array}$




$\begin{array}{lll}\text { H } 5.16970900 & -2.18182400 & 1.10776500 \\ \text { H } 6.14098700 & -3.53996900 & 1.46025300 \\ \text { H } 4.61892300 & 2.61565100 & 1.12466700 \\ \text { H } 4.38647600 & 0.92968400 & 1.22641000 \\ \text { H } 6.13397200 & -2.88807000 & -0.08111400 \\ \text { H } 3.04681700 & 2.01836300 & 1.25194700 \\ \text { Au -0.64351100 } & 1.62937400 & 0.07873400 \\ \text { Au -2.39421800 } & -0.63820500 & 0.00730600 \\ \text { Au -3.25333500 } & 1.94433800 & 0.18254000 \\ \text { Au -2.27832400 } & -3.24398900 & -0.15008300 \\ \text { H= -1208.196753 Hartree } & \\ \text { G= -1208.290890 Hartree } & \end{array}$

Structure 9

\begin{tabular}{|c|c|c|}
\hline S - 2.98848500 & 0.69640000 & -1.53666900 \\
\hline S -4.46201900 & 2.44673500 & -1.43894600 \\
\hline O -3.98060100 & -2.30912900 & 2.64230300 \\
\hline O - 0.49920000 & 3.15537300 & 0.04291600 \\
\hline O -5.73532200 & -2.11742800 & 1.13835200 \\
\hline O - 1.85622200 & 2.05647700 & 1.55301500 \\
\hline N - 2.41293200 & -0.61956100 & 1.45600400 \\
\hline$N-2.30731500$ & 4.78324300 & -0.84999500 \\
\hline C - 3.82797700 & -0.61906400 & 0.91546800 \\
\hline C -2.73291500 & 3.98071700 & 0.36894100 \\
\hline C - -3.89563300 & -0.70485300 & -0.60909600 \\
\hline C -4.12460400 & 3.34662300 & 0.20995700 \\
\hline C -4.59301400 & -1.80440100 & 1.61820300 \\
\hline C - 1.61621200 & 2.95241100 & 0.69226400 \\
\hline$H-4.30016000$ & 0.30655300 & 1.26045100 \\
\hline H - 2.77622500 & 4.67204400 & 1.21772700 \\
\hline$H-3.40685000$ & -1.60881800 & -0.99129800 \\
\hline H -4.93743300 & -0.70530700 & -0.92831800 \\
\hline H -4.29492500 & 2.66786900 & 1.04429100 \\
\hline$H-4.90605800$ & 4.11456900 & 0.21144500 \\
\hline H - 2.49391800 & -1.14804600 & 2.35262500 \\
\hline H - 2.05418500 & 0.35278200 & 1.58838300 \\
\hline H -1.75793600 & -1.14662300 & 0.84693100 \\
\hline H -1.27774000 & 4.67057700 & -0.92971000 \\
\hline H - 2.74348400 & 4.39505800 & -1.70233700 \\
\hline H - 2.55602400 & 5.77591400 & -0.77318500 \\
\hline Au 1.16966700 & 1.77044500 & 0.13722500 \\
\hline Au 1.15622700 & -1.07047500 & -0.14273200 \\
\hline Au 3.41979400 & 0.41997100 & 0.19619000 \\
\hline $\mathrm{Au}-0.54230100$ & $-3.0309970 c$ & $\begin{array}{ll}0 & -0.46937100\end{array}$ \\
\hline
\end{tabular}

$G=-1208.290214$ Hartree

Structure 10

S $3.77786400-1.09955800-0.69794700$

S $5.65145000-2.38953800-0.54107200$

$\begin{array}{llll}0 & 1.64839900 & 1.68874100 & -0.56283300\end{array}$

$\begin{array}{llll}0 & 6.47121300 & 0.94522300 & -0.07934900\end{array}$

$\begin{array}{llll}0 & 1.49256100 & 1.30658500 & -2.83981500\end{array}$

$\begin{array}{llll}0 & 7.82775700 & 1.38302600 & 1.73661200\end{array}$

N $4.22545800 \quad 2.17678500-0.56094400$

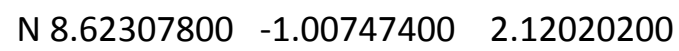

C $3.68776700 \quad 1.54563700-1.82929200$

$\begin{array}{lll}\text { C } 7.67565000 & -0.89262500 & 0.92058000\end{array}$

$\begin{array}{llll}C & 4.28265100 & 0.14491500 & -2.06755500\end{array}$

C $6.47310100-1.84768700 \quad 1.08866800$

$\begin{array}{llll}\text { C } 2.13806400 & 1.51604200 & -1.77834300\end{array}$

$\begin{array}{lll}\text { C } 7.28887500 & 0.61973400 & 0.85524100\end{array}$

H $3.98624900 \quad 2.16986300-2.67677400$

H $8.24420500 \quad-1.15577600 \quad 0.02516800$

H $3.91572200-0.24220100-3.01868200$

$\begin{array}{lll}\text { H } 5.37202100 & 0.18374600 & -2.06067000\end{array}$

H $6.80860200-2.801549001 .51057700$

H $5.69846500-1.430119001 .73551400$

H $3.532867001 .99941100 \quad 0.18446500$

H $4.309466003 .19309800-0.67101700$

H $8.72510500-0.00648500 \quad 2.43662400$

H $9.53418100-1.40078200 \quad 1.86235300$

H $5.183165001 .76839600-0.29537000$

$\begin{array}{lll}\text { H } 8.23208900 & -1.56653600 & 2.88692900\end{array}$

Au $-0.45985300 \quad 1.61569300-0.13916700$

Au -2.96468700 $2.06459000 \quad 0.53851400$

$\mathrm{Au}-2.31069700 \quad-0.55217000 \quad 0.14255900$

Au -2.40586400 -3.15565100 -0.06320200

$\mathrm{H}=-1208.193311$ Hartree

$\mathrm{G}=-1208.290278$ Hartree

Structure 11

S -7.32233100 $2.32848600 \quad 0.40139900$

S -6.18327000 $1.55133800-1.43068000$

$\begin{array}{llll}0 & -7.73890600 & -1.75282400 & -0.40516900\end{array}$

$\begin{array}{llll}0 & -1.68323700 & -1.00730400 & -0.90774200\end{array}$

$\begin{array}{llll}0 & -5.80079900 & -1.26893400 & 0.76463700\end{array}$

$\begin{array}{llll}0 & -1.53593900 & 1.29647500 & -1.03601300\end{array}$

N-6.91659100 $0.04486500 \quad 2.66821200$

N -4.22264900 $-1.18990400-1.34505500$ 


$\begin{array}{lrr}\text { C-7.87964700 } & -0.32090200 & 1.54604800 \\ \text { C-3.66926600 } & 0.21278800 & -1.51654200 \\ \text { C-8.51129800 } & 0.91237400 & 0.87523800 \\ \text { C-4.43369400 } & 1.26552800 & -0.69809900 \\ \text { C-7.09397900 } & -1.20516500 & 0.53231100 \\ \text { C-2.16148200 } & 0.20667500 & -1.11131600 \\ \text { H-8.68826100 } & -0.92213100 & 1.97113200 \\ \text { H-3.69829800 } & 0.46388000 & -2.58292300 \\ \text { H-9.05444100 } & 0.59325000 & -0.01505000 \\ \text { H-9.20709100 } & 1.41766500 & 1.55451800 \\ \text { H-4.54993500 } & 0.95311500 & 0.33985800 \\ \text { H-3.89452700 } & 2.21043000 & -0.74852200 \\ \text { H-4.77384600 } & -1.48490100 & -2.15667800 \\ \text { H-4.84483400 } & -1.28696300 & -0.46407800 \\ \text { H-3.39427800 } & -1.80566300 & -1.24091800 \\ \text { H-7.23649300 } & -0.28420300 & 3.58484900 \\ \text { H-6.02189400 } & -0.42944200 & 2.39415000 \\ \text { H-6.74532600 } & 1.05876000 & 2.71316100 \\ \text { Au 3.20970200 } & 2.89184800 & 0.14730600 \\ \text { Au } 0.36979400 & -1.37079300 & -0.35925800 \\ \text { Au 2.60044100 } & 0.35208000 & 0.12453200 \\ \text { Au 2.71551700 } & -2.36823400 & 0.29964000 \\ \text { H=-1208.196725 Hartree } & \\ \text { G=-1208.289840 Hartree } & \end{array}$

Structure 12

\begin{tabular}{|c|c|c|}
\hline 46903 & -0.71583800 & -1.44 \\
\hline$S-6.13028700$ & 1.04831600 & -1.01484000 \\
\hline O -1.68506100 & -3.12255400 & 0.41630300 \\
\hline O -3.56274800 & 0.97109600 & 1.36630400 \\
\hline O -3.08709700 & -3.26624000 & -1.41963300 \\
\hline O -2.07142100 & 2.44396500 & 0.39244100 \\
\hline N -3.43315700 & -1.66799800 & 1.81270800 \\
\hline N -3.78720100 & 4.37844600 & 0.18823900 \\
\hline C -4.01322100 & -2.54233900 & 0.71427100 \\
\hline C -4.40380100 & 2.98327900 & 0.32109700 \\
\hline C -5.19439200 & -1.89879900 & -0.02156200 \\
\hline C -4.99446500 & 2.56633100 & -1.04671700 \\
\hline C -2.84125200 & -3.00593200 & -0.20795000 \\
\hline C -3.26046800 & 2.03788000 & 0.75794300 \\
\hline H -4.38925400 & -3.44562200 & 1.21162900 \\
\hline H -5.18291800 & 3.02171000 & 1.08387900 \\
\hline H -5.78881700 & -2.68340400 & -0.48982200 \\
\hline H -5.83027500 & -1.33226000 & 0.66301600 \\
\hline$H-5.66544800$ & 3.34991300 & -1.41543200 \\
\hline
\end{tabular}

H $-4.21211800 \quad 2.40098700 \quad-1.79163300$

$\begin{array}{lll}H-2.46924200 & -2.00790400 & 1.97252100\end{array}$

$\begin{array}{lll}H-3.97159000 & -1.75556900 & 2.68133400\end{array}$

$\begin{array}{lll}H-3.39458500 & -0.64182700 & 1.55870300\end{array}$

H $-4.28139300 \quad 4.97074000 \quad-0.48966600$

H -3.78167500 $4.86492800 \quad 1.09351100$

H - $2.80097100 \quad 4.23540400-0.10409000$

Au $-0.37735100 \quad 1.03976700 \quad 0.29369100$

Au -0.05057600 -1.62053000 0.14462500

Au $2.12453100 \quad-0.02120800-0.02433400$

Au $4.72031800 \quad 0.44598900-0.28453500$

$\mathrm{H}=-1208.197538$ Hartree

$\mathrm{G}=-1208.289436$ Hartree

Structure 13

S -5.87217300 $0.89286800-1.97391600$

S - $4.01709000-0.11563900-1.09113100$

$\begin{array}{llll}0-5.18887700 & 1.29618600 & 2.29437800\end{array}$

$\begin{array}{llll}0 & -1.49118100 & -2.04250600 & 0.43541700\end{array}$

$\begin{array}{llll}0-6.01054100 & -0.74193700 & 1.56841600\end{array}$

O - $1.80827500 \quad-3.30536500-1.47741500$

N-6.55482400 $2.73691100 \quad 0.62665700$

N-3.92183800 $-2.31991800 \quad 1.35288800$

$\begin{array}{llll}\text { C }-7.00869500 & 1.28914500 & 0.70336200\end{array}$

$\begin{array}{lll}\text { C }-3.72464200 & -2.77299500 & -0.08259700\end{array}$

C - $7.23996700 \quad 0.64719000 \quad-0.66872900$

$\begin{array}{llll}C-4.56133500 & -1.95228400 & -1.08276300\end{array}$

$\begin{array}{lll}\text { C }-5.96321100 & 0.54871300 & 1.60077800\end{array}$

$\begin{array}{lll}\text { C }-2.21168400 & -2.73168000 & -0.43173700\end{array}$

$\mathrm{H}-7.96138800 \quad 1.27280000 \quad 1.24439600$

$\begin{array}{lll}H-4.05938600 & -3.81083500 & -0.16681800\end{array}$

$\begin{array}{lll}H-7.40833000 & -0.41972800 & -0.52317500\end{array}$

H -8.11142300 $1.07883800-1.17335500$

H $-4.42577300 \quad-2.35617700 \quad-2.08626400$

$\begin{array}{lll}H-5.61509400 & -1.98144300 & -0.80098700\end{array}$

$\begin{array}{lll}H-7.32855800 & 3.40645800 & 0.69194000\end{array}$

H $-6.03484200 \quad 2.90774100-0.24777900$

H $-4.02126800 \quad-3.13014900 \quad 1.97435300$

$\begin{array}{lll}H-4.78049200 & -1.67474100 & 1.49210900\end{array}$

$\begin{array}{lll}H-5.89586500 & 2.84291600 & 1.43268200\end{array}$

H -3.05745700 $-1.81649000 \quad 1.62142900$

Au $0.61957900 \quad-1.69721000 \quad 0.18183600$

Au $2.07526100 \quad 0.76559600 \quad-0.00222500$

Au $3.24432500-1.69806600-0.00203600$

Au $1.66226400 \quad 3.34495400 \quad-0.06454800$ 
$H=-1208.196626$ Hartree

$\mathrm{G}=-1208.289571$ Hartree

Structure 14

S $7.36778500-1.41380200 \quad 1.02753900$

S $6.25113200-1.30815100-0.97231300$

$\begin{array}{llll}0 & 5.69187000 & 2.03241600 & 0.16794300\end{array}$

$\begin{array}{llll}0 & 1.57784100 & 0.99670900 & -1.30561900\end{array}$

$\begin{array}{llll}0 & 7.65614700 & 2.21376200 & -1.04366300\end{array}$

$\begin{array}{llll}0 & 1.64002400 & -1.25810600 & -0.79732600\end{array}$

$\begin{array}{lll}\text { N } 6.77711100 & 1.44856100 & 2.42000400\end{array}$

N $4.15158400 \quad 1.20860300-1.80110100$

$\begin{array}{lll}\text { C } 7.77132100 & 1.48597500 & 1.26611800\end{array}$

C $3.68307900-0.20699900-1.52172800$

$\begin{array}{llll}\text { C } 8.47878800 & 0.13702400 & 1.04183800\end{array}$

$\begin{array}{llll}C & 4.46994700 & -0.89129400 & -0.39285400\end{array}$

$\begin{array}{lll}\text { C } 6.99473400 & 1.96116200 & 0.00172600\end{array}$

C $2.16281800-0.12243000-1.17439500$

$\begin{array}{lll}\text { H } 8.53728000 & 2.23115800 & 1.49753800\end{array}$

H $3.76092400-0.78625000-2.44822000$

$\begin{array}{lll}\text { H } 9.03761500 & 0.18137700 & 0.10634600\end{array}$

$\begin{array}{lll}\text { H } 9.17225200 & -0.08636100 & 1.86017600\end{array}$

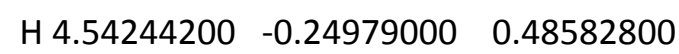

$\begin{array}{llll}\text { H } 3.97781700 & -1.82836400 & -0.13842600\end{array}$

$\begin{array}{lll}\text { H } 5.87716300 & 1.76744800 & 1.98542500\end{array}$

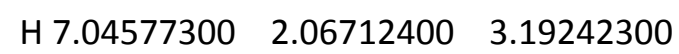

H $4.67902500 \quad 1.27157100-2.67700800$

H $4.768570001 .60915400-1.00474000$

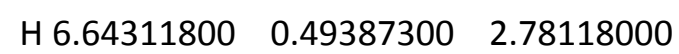

H $3.288250001 .78175400-1.87395100$

Au -0.44548700 $-1.47120600 \quad-0.28575400$

$\mathrm{Au}-2.56937000 \quad 0.40687000 \quad 0.11617200$

$\begin{array}{lll}\mathrm{Au}-2.86006100 & -2.29553300 & 0.35689100\end{array}$

$\begin{array}{lll}\text { Au }-2.98109300 & 2.98568600 & 0.05981200\end{array}$

$H=-1208.197104$ Hartree

$G=-1208.289456$ Hartree

Structure 15

$\begin{array}{lll}\text { S } 7.21023100 & 2.35405400 & 0.27510600 \\ \text { S 4.93568100 } & 2.09972700 & 0.43447600 \\ \text { O } 6.71724200 & -1.58460800 & -0.80719000 \\ \text { O } 1.87712400 & 0.88983400 & 0.10132900 \\ \text { O } 6.20286900 & -1.41977200 & 1.44073800 \\ \text { O } 1.67462400 & -1.32473900 & -0.54122500 \\ \text { N } 8.87830900 & -0.20554900 & -0.73671000\end{array}$

N $4.15846600-1.49767500 \quad-1.48364900$

C $8.23257000 \quad-0.36707200 \quad 0.63215000$

C $3.86156500-0.34737700-0.53665200$

$\begin{array}{lll}\text { C } 7.98613500 & 0.97632000 & 1.34590300\end{array}$

C $4.52042500 \quad 0.95161100-1.03348900$

C $6.93435800-1.20151300 \quad 0.42860700$

C $2.34218600-0.20947700-0.31083300$

H $8.91429500-0.95592000 \quad 1.25406100$

H $4.30584100 \quad-0.64926200 \quad 0.41993600$

$\begin{array}{lll}\text { H } 7.35219800 & 0.80509000 & 2.21677500\end{array}$

H $8.92985500 \quad 1.42544200 \quad 1.67123400$

H $5.46127700 \quad 0.74907800-1.54879700$

H $3.859467001 .51812500-1.69167700$

H $8.34357000-0.86440500-1.35078500$

H $9.87923400-0.42607600-0.73463000$

H $4.00202300-1.24091200-2.46509700$

H $3.51673400 \quad-2.27043700-1.25589400$

H $8.74062600 \quad 0.74793100-1.10322000$

H $5.18450700-1.76104700-1.32552200$

Au -0.43884300 - $-1.49110900-0.17194000$

Au-2.56462700 $0.41668500 \quad 0.03902200$

$\begin{array}{lll}\text { Au }-2.91107800 & -2.27640000 & 0.28312100\end{array}$

Au -2.93507200 $2.99998600 \quad-0.08185500$

$\mathrm{H}=-1208.195128$ Hartree

$\mathrm{G}=-1208.289329$ Hartree

Structure 16

S $6.53663600-0.15240400 \quad 1.01882100$

S $4.42384700-0.88388200 \quad 1.50761700$

$\begin{array}{llll}0 & 3.26291100 & 1.88716600 & -0.98862200\end{array}$

$\begin{array}{llll}0 & 1.08568200 & -2.63394700 & -0.41633500\end{array}$

O $3.81135100-0.17734000-1.86798300$

$\begin{array}{llll}0 & 1.74702000 & -2.45525000 & 1.79309400\end{array}$

N $5.68561400 \quad 2.60511100-0.43074200$

N $3.53865600-2.73856100-1.33776100$

C $5.60022800 \quad 1.36267800-1.30146300$

$\begin{array}{llll}\text { C } 3.35946800 & -3.16915700 & 0.10428900\end{array}$

$\begin{array}{llll}\text { C } 6.53585500 & 0.23645100 & -0.84680700\end{array}$

$\begin{array}{lll}\text { C } 4.49562400 & -2.73875500 & 1.03876200\end{array}$

$\begin{array}{llll}\text { C } 4.08209200 & 0.97922000 & -1.37575000\end{array}$

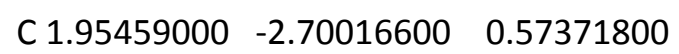

H $5.89497700 \quad 1.65449300-2.31595600$

H $3.33977100-4.26721400 \quad 0.09799100$

H $6.29588300-0.66723800-1.40652000$

H $7.58593000 \quad 0.49474200-1.02415000$ 


$$
\begin{array}{lll}
\text { H } 4.40383600 & -3.28771800 & 1.97657900 \\
\text { H } 5.47846700 & -2.93712200 & 0.60541400 \\
\text { H } 6.33350300 & 3.31056500 & -0.79547300 \\
\text { H } 5.96486100 & 2.35428900 & 0.53048500 \\
\text { H } 4.30547500 & -3.24853300 & -1.78937100 \\
\text { H } 3.69883100 & -1.68549900 & -1.45993600 \\
\text { H } 4.70331800 & 2.96772900 & -0.41291600 \\
\text { H 2.65074100 } & -2.94968600 & -1.81869600 \\
\text { Au -0.69117100 } & 3.28375900 & 0.21670700 \\
\text { Au -0.89608500 } & -1.82912300 & -0.17910100 \\
\text { Au -1.74345900 } & 0.89684900 & 0.06766600 \\
\text { Au -3.45429800 } & -1.22503900 & -0.07424300 \\
\text { H= -1208.197190 Hartree } & \\
\text { G= -1208.289349 Hartree } &
\end{array}
$$

\begin{tabular}{|c|c|c|}
\hline S -7.08776900 & 1.01407400 & 1.39622600 \\
\hline S -5.84511000 & -0.38188100 & 2.71464300 \\
\hline O -7.70381000 & -1.72838800 & -3.00944600 \\
\hline O -1.65480000 & -1.48840900 & -0.10237100 \\
\hline O -6.10211100 & -2.12702800 & -1.39970700 \\
\hline O -1.82985600 & 0.75409500 & 0.44031400 \\
\hline N -8.40153100 & 0.60080400 & -2.20212000 \\
\hline N -4.18146400 & -2.05162900 & 0.41492000 \\
\hline C - 7.36713600 & -0.05358400 & -1.29097300 \\
\hline C -3.79445400 & -0.60716600 & 0.65254400 \\
\hline C - 7.86851800 & -0.22452000 & 0.15920700 \\
\hline C -4.06398200 & -0.12880400 & 2.08704200 \\
\hline C - 7.02686800 & -1.43353100 & -1.96118200 \\
\hline C - 2.28730700 & -0.46084100 & 0.29221000 \\
\hline H -6.46570100 & 0.56205200 & -1.31676800 \\
\hline H -4.36195400 & 0.01165100 & -0.04904000 \\
\hline H -8.95057800 & -0.09444500 & 0.24556100 \\
\hline H -7.61582700 & -1.21789000 & 0.53199000 \\
\hline H -3.46930700 & -0.68507800 & 2.82173700 \\
\hline H -3.81758900 & 0.92967800 & 2.16337800 \\
\hline H -9.26806200 & 0.85635200 & -1.71675700 \\
\hline H -8.59258700 & -0.14761900 & -2.92027500 \\
\hline H -4.95804500 & -2.11008600 & -0.31994000 \\
\hline H -4.51400100 & -2.50933100 & 1.27124300 \\
\hline H -8.03540000 & 1.43680800 & -2.67046500 \\
\hline H -3.32329900 & -2.53082900 & 0.07924900 \\
\hline Au 0.23148200 & 1.27994100 & 0.08473900 \\
\hline Au 2.64712400 & -0.25162800 & -0.06669200 \\
\hline Au 2.54869000 & 2.46234300 & -0.31112400 \\
\hline Au 3.44806600 & -2.73485900 & 0.05957200 \\
\hline \multicolumn{3}{|c|}{$H=-1208.195233$ Hartree } \\
\hline \multicolumn{3}{|c|}{$=-1208.288791$ Hartree } \\
\hline
\end{tabular}

Structure 17

$$
\begin{array}{lrr}
\text { S } 6.39672500 & -1.21127700 & 1.99918600 \\
\text { S 7.38079700 } & -1.03136800 & -0.05082700 \\
\text { O } 1.80767900 & -1.62178400 & 2.17013100 \\
\text { O } 5.39350500 & 3.21759900 & -0.98194800 \\
\text { O } 1.57933900 & -1.71010700 & -0.12823700 \\
\text { O } 4.70384200 & 1.02806800 & -0.87277300 \\
\text { N } 4.09958600 & -1.52636000 & -0.68542400 \\
\text { N 7.76818400 } & 2.79323800 & -1.77756500 \\
\text { C } 3.80813800 & -1.78662100 & 0.78210400 \\
\text { C 7.04448000 } & 1.50037300 & -1.38963400 \\
\text { C } 4.55744300 & -0.82134100 & 1.70928600 \\
\text { C 7.79259300 } & 0.82274500 & -0.21607100 \\
\text { C } 2.26691900 & -1.68401500 & 0.99933800 \\
\text { C } 5.58208100 & 1.94768700 & -1.05168400 \\
\text { H 4.08826200 } & -2.82159300 & 1.00729100 \\
\text { H 7.03708900 } & 0.84163100 & -2.26068500 \\
\text { H } 4.46622000 & 0.21209300 & 1.37274800 \\
\text { H 4.13906500 } & -0.91450700 & 2.71664500 \\
\text { H } 8.87096200 & 0.84147200 & -0.39966700 \\
\text { H 7.58582600 } & 1.30193700 & 0.74361400 \\
\text { H } 4.83870800 & -2.13730400 & -1.04524900 \\
\text { H } 4.39776800 & -0.51020100 & -0.84085800 \\
\text { H 7.95904300 } & 2.83882800 & -2.78444900 \\
\text { H 8.65064800 } & 2.93289800 & -1.27356100 \\
\text { H 3.21049400 } & -1.69989000 & -1.18810600 \\
\text { H 7.06947200 } & 3.53967100 & -1.51544400 \\
\text { Au -0.57048300 } & -1.60660400 & -0.17961000 \\
\text { Au -3.16341300 } & -1.97601800 & -0.44056300 \\
\text { Au -2.38635900 } & 0.59713800 & 0.02064200 \\
& & \\
\hline
\end{array}
$$

Au -2.36092400 $3.18049500 \quad 0.41013100$ $\mathrm{H}=-1208.194684$ Hartree

$G=-1208.288729$ Hartree

Structure 18

Structure 19

$\begin{array}{lcc}\text { S } 4.13999900 & 2.58809300 & -1.69228600 \\ \text { S 5.83094500 } & 1.83632200 & -0.35244800 \\ \text { O }-0.15162300 & 2.81602400 & -0.04479100 \\ \text { O } 3.51393000 & -2.02148400 & 0.15203900 \\ \text { O } 0.50000200 & 2.49985400 & 2.15228400 \\ \text { O } 3.69056500 & -0.05720800 & 1.33687200\end{array}$




\begin{tabular}{|c|c|c|}
\hline N 3.06906600 & 2.53740000 & 1.65947300 \\
\hline & & 400 \\
\hline C 2.20658300 & 2.94795500 & 0.47708600 \\
\hline C 5.68127500 & -0.97931100 & 0.29600600 \\
\hline C 2.51850700 & 2.15128500 & -0.79551500 \\
\hline C 6.02311700 & 0.00636400 & -0.85068600 \\
\hline C 0.72260000 & 2.73064000 & 0.92286300 \\
\hline C 4.16956500 & -1.00846300 & 0.64488300 \\
\hline H 2.35482200 & 4.01802200 & 0.30255700 \\
\hline H 6.26302400 & -0.73765900 & 1.18755800 \\
\hline H 2.47910500 & 1.07454000 & -0.62259800 \\
\hline H 1.76776500 & 2.40975600 & -1.54833900 \\
\hline H 7.07787000 & -0.09473500 & -1.12418200 \\
\hline H 5.40359000 & -0.16491300 & -1.73469700 \\
\hline H 3.88917300 & 3.14082500 & 1.77254400 \\
\hline H 3.38831100 & 1.53463100 & 1.57227700 \\
\hline H 6.43666800 & -2.94316000 & 0.63532900 \\
\hline H 6.72095600 & -2.42255800 & -0.91844000 \\
\hline H 2.45388000 & 2.60435900 & 2.49346700 \\
\hline H 5.14456000 & -2.83831700 & -0.43284200 \\
\hline Au 1.22448900 & -1.91671700 & 0.08774000 \\
\hline $\mathrm{Au}-1.9458540 \mathrm{C}$ & $0 \quad 1.57939500$ & $0-0.03237500$ \\
\hline $\mathrm{Au}-1.27553300$ & $0-1.25458900$ & $0-0.04866300$ \\
\hline $\mathrm{Au}-3.89014800$ & $0-0.1589780 c$ & $0 \quad-0.16377800$ \\
\hline \multicolumn{3}{|c|}{$H=-1208.199617$ Hartree } \\
\hline \multicolumn{3}{|c|}{$G=-1208.288278$ Hartree } \\
\hline
\end{tabular}

Structure 20

$\begin{array}{lrr}\text { S } 4.78853200 & -0.23367000 & 1.57072200 \\ \text { S } 2.86631900 & -1.37748800 & 2.10675700 \\ \text { O } 4.75185000 & -0.99331600 & -1.85914600 \\ \text { O } 1.66995700 & -1.79065600 & -1.25304300 \\ \text { O } 6.30794000 & 0.69392000 & -1.66389200 \\ \text { O } 0.45457100 & -3.11342800 & 0.18038500 \\ \text { N } 2.66998200 & 0.71675200 & -1.41507900 \\ \text { N } 3.92892100 & -3.27858500 & -0.95938100 \\ \text { C } 4.05067200 & 1.14851200 & -0.95557400 \\ \text { C } 2.81216900 & -3.50454900 & 0.03967800 \\ \text { C } 4.15968400 & 1.27222300 & 0.57931800 \\ \text { C } 3.21159800 & -3.14811900 & 1.48986000 \\ \text { C } 5.13774100 & 0.20978000 & -1.55171800 \\ \text { C } 1.55415300 & -2.73445700 & -0.40708600 \\ \text { H } 4.20762300 & 2.15168600 & -1.36350500 \\ \text { H } 2.58711200 & -4.57559600 & 0.02264600 \\ \text { H } 4.92605400 & 2.01756400 & 0.81099600\end{array}$

H 3.219800001 .605952001 .02900100

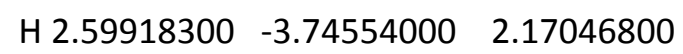
H $4.26072400-3.380231001 .68963000$ H $2.47540000-0.30896800-1.27957200$ H $2.57618400 \quad 0.89518600-2.42263200$ H $3.68274000-3.72530700-1.85150500$ H $4.79739300-3.71551000-0.62868100$ H $1.924581001 .26319500-0.93431200$ H $4.14897500 \quad-2.24579900-1.20109700$ Au -1.29100200 $-1.82284600 \quad 0.00353900$ $\mathrm{Au}-3.52614200-0.45508200-0.16148000$ $\mathrm{Au}-1.24286600 \quad 1.03984400 \quad 0.02199900$ Au $0.47638500 \quad 3.00570600 \quad 0.17820600$ $\mathrm{H}=-1208.198040$ Hartree $\mathrm{G}=-1208.288360$ Hartree

Structure 21

$\begin{array}{lrc}\text { S } 2.86910300 & -1.37593800 & 2.10788200 \\ \text { S } 4.79083800 & -0.23315600 & 1.56796700 \\ \text { O } 1.66909200 & -1.79028300 & -1.25037600 \\ \text { O } 4.75022300 & -0.99473600 & -1.86129100 \\ \text { O } 0.45436900 & -3.11274600 & 0.18378800 \\ \text { O } 6.30700000 & 0.69217200 & -1.66871800 \\ \text { N } 3.92794100 & -3.27899400 & -0.95810300 \\ \text { N } 2.66933900 & 0.71624100 & -1.41639100 \\ \text { C } 2.81192300 & -3.50409900 & 0.04199000 \\ \text { C } 4.05056100 & 1.14783400 & -0.95835800 \\ \text { C } 3.21266100 & -3.14706300 & 1.49166100 \\ \text { C } 4.16104600 & 1.27228700 & 0.57639500 \\ \text { C } 1.55373700 & -2.73395400 & -0.40419600 \\ \text { C } 5.13678000 & 0.20843800 & -1.55504600 \\ \text { H 2.58657900 } & -4.57509700 & 0.02573300 \\ \text { H } 4.20748100 & 2.15074500 & -1.36694500 \\ \text { H } 2.60028100 & -3.74364400 & 2.17305700 \\ \text { H } 4.26174200 & -3.37993000 & 1.69078800 \\ \text { H } 4.92763900 & 2.01772800 & 0.80698500 \\ \text { H } 3.22160400 & 1.60619500 & 1.02687100 \\ \text { H } 4.14748000 & -2.24646600 & -1.20118300 \\ \text { H } 3.68137500 & -3.72692400 & -1.84951300 \\ \text { H 2.57468000 } & 0.89413500 & -2.42396300 \\ \text { H } 1.92453100 & 1.26305200 & -0.93520700 \\ \text { H } 4.79681100 & -3.71515200 & -0.62743800 \\ \text { H } 2.47458000 & -0.30938100 & -1.28006900 \\ \text { Au }-1.29164600 & -1.82292600 & 0.00389500 \\ \text { Au }-3.52633300 & -0.45475100 & -0.16293700\end{array}$


$\mathrm{Au}-1.24318200 \quad 1.04004700 \quad 0.02282500$

Au $0.47697800 \quad 3.00514400 \quad 0.17908900$

$\mathrm{H}=-1208.198034$ Hartree

$G=-1208.288315$ Hartree

\section{Structure 22}

S - $4.45879700-1.00528400-1.21150200$

S -6.52966000 $-0.53249500-2.03918600$

$\begin{array}{llll}0-2.17760500 & -3.41138200 & 0.95234200\end{array}$

$\begin{array}{llll}0-6.93397000 & 2.76465300 & 1.57889000\end{array}$

$\begin{array}{llll}0 & -1.65145800 & -1.15928700 & 0.90852000\end{array}$

$\begin{array}{llll}0-6.22721700 & 0.59055400 & 1.25292400\end{array}$

N -3.90670400 $-0.34839300 \quad 1.97661600$

$\begin{array}{llll}N-8.37400700 & 2.94208300 & -0.51903800\end{array}$

C-3.89703100 $-1.78686900 \quad 1.49983800$

C $-7.67745400 \quad 1.58213200-0.40021900$

$\begin{array}{lll}\text { C }-4.90322700 & -2.01661600 & 0.35658400\end{array}$

C $-6.80357800 \quad 1.30952100-1.64552300$

$\begin{array}{lll}\text { C }-2.45568700 & -2.19045300 & 1.09581800\end{array}$

C $-6.87125900 \quad 1.65482300 \quad 0.93565100$

H $-4.20540100 \quad-2.42707200 \quad 2.33193500$

$\mathrm{H}-8.45342100 \quad 0.81861000 \quad-0.31124300$

$\begin{array}{lll}H-4.90017000 & -3.07245200 & 0.08393400\end{array}$

$\begin{array}{lll}H-5.90160300 & -1.69633500 & 0.65472200\end{array}$

$\begin{array}{llll}H-7.31537200 & 1.66322700 & -2.54730300\end{array}$

H -5.82676900 $1.79402500-1.58385200$

H-3.13018300 $0.13641700 \quad 1.49855300$

$\begin{array}{lll}H-3.72716400 & -0.30177700 & 2.98529300\end{array}$

$\begin{array}{llll}H-9.39571000 & 2.85527000 & -0.53739100\end{array}$

$\mathrm{H}-8.07867500 \quad 3.47661400 \quad-1.34376300$

H-4.84490800 $0.12926300 \quad 1.76249400$

$\begin{array}{lll}H-8.06328200 & 3.44369600 & 0.35533600\end{array}$

$\begin{array}{lll}\text { Au } 0.40513400 & -1.40629800 & 0.31857500\end{array}$

$\begin{array}{lll}\text { Au } 2.84196200 & -2.10385900 & -0.39347700\end{array}$

Au $2.44598000 \quad 0.57296300 \quad-0.08602900$

Au $2.75242200 \quad 3.16551800 \quad 0.03263900$

$\mathrm{H}=-1208.193191$ Hartree

$G=-1208.288505$ Hartree

Structure 23

S $-6.02156200 \quad-2.36154100 \quad 0.15012800$

S -7.41477300 $-0.80276500 \quad 1.06103900$

$\begin{array}{llll}0-1.52945300 & -1.66920000 & 0.43420700\end{array}$

$\begin{array}{llll}0-6.36366600 & 2.46674400 & -2.25909300\end{array}$

$\begin{array}{llll}0-1.68442700 & 0.38748600 & 1.47735800\end{array}$

\begin{abstract}
O $-5.31073400 \quad 1.51166300-0.45309500$
$\begin{array}{lll}\mathrm{N}-4.30520800 & 0.35213200 & 1.68101500\end{array}$

N-8.73693000 $2.39032000-1.35077900$

$\begin{array}{lll}\text { C }-3.69686900 & -0.95672300 & 1.20995200\end{array}$

C - $7.75055800 \quad 1.61387500-0.47346500$

$\begin{array}{lll}\text { C }-4.35058300 & -1.47751400 & -0.07608800\end{array}$

$\begin{array}{llll}C-8.14696900 & 0.11811400 & -0.43870600\end{array}$

C - $2.16953000-0.69582500 \quad 1.02622600$

C - $-6.34756500 \quad 1.88699200-1.11183500$

$\begin{array}{lll}\mathrm{H}-3.79927400 & -1.69483600 & 2.01245300\end{array}$

$\begin{array}{lll}H-7.78948600 & 2.03551000 & 0.53298300\end{array}$

H-4.46973400 $-0.68618000-0.81714300$

$\begin{array}{lll}H-3.72356400 & -2.27487100 & -0.48630000\end{array}$

$\begin{array}{lll}H-9.22860900 & 0.01818400 & -0.30901100\end{array}$

H -7.84595600 $-0.41469600 \quad-1.34353400$

$\begin{array}{lll}H-4.99043700 & 0.21279300 & 2.42911300\end{array}$

$\begin{array}{lll}H-4.78623300 & 0.86485700 & 0.87223200\end{array}$

H $-9.09164800 \quad 3.22743400-0.87525000$

$\begin{array}{llll}H-9.53798800 & 1.83014200 & -1.66220300\end{array}$

H -3.51320900 $0.92967600 \quad 2.02167100$

H -8.14249000 $2.67851400 \quad-2.17350100$

$\begin{array}{lll}\text { Au } 0.59724700 & -1.59842300 & 0.07514700\end{array}$

$\begin{array}{llll}\text { Au } 3.12613100 & -2.07693500 & -0.47363200\end{array}$

$\begin{array}{lll}\text { Au } 2.49285000 & 0.54191000 & -0.04833000\end{array}$

$\begin{array}{lll}\text { Au } 2.60749000 & 3.13595600 & 0.24044400\end{array}$

$\mathrm{H}=-1208.194913$ Hartree

$\mathrm{G}=-1208.288039$ Hartree
\end{abstract}

Structure 24

S $7.25072300-1.144917001 .61165400$

S $5.86031300-2.71461500 \quad 0.64681200$

$\begin{array}{llll}0 & 8.33932500 & 2.13963600 & -1.42147600\end{array}$

$\begin{array}{llll}0 & 1.78204000 & 0.66818900 & 0.31464600\end{array}$

$\begin{array}{llll}0 & 6.41706100 & 1.58745700 & -0.28722800\end{array}$

$01.51455400-1.57094000-0.19400700$

N $8.93617400 \quad-0.22178000-2.18545800$

N $4.31227000 \quad 0.55396100 \quad 0.86028700$

C $7.87241100-0.20420900-1.08566900$

C $3.76054500 \quad-0.70264200 \quad 0.21949500$

$\begin{array}{lll}\text { C } 8.46310500 & -0.85419900 & 0.18397200\end{array}$

C $4.13539000-1.98583000 \quad 0.97082300$

$\begin{array}{llll}C & 7.50268500 & 1.30573100 & -0.91338900\end{array}$

C $2.20823700 \quad-0.56371800 \quad 0.10627700$

H $7.01099700-0.77723200-1.43369100$

H $4.14074800-0.74238300-0.80701600$ 


\begin{abstract}
H $8.89245400-1.82959000-0.05986600$ H $9.24230100-0.22951400 \quad 0.63573200$ H $4.00065300 \quad-1.88290800 \quad 2.05105800$ $\begin{array}{lll}\text { H } 3.47926500 & -2.78949800 & 0.62098400\end{array}$

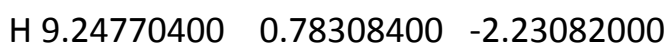
H $8.53837300-0.49222800-3.09220800$ H $3.56005000 \quad 1.26336600 \quad 0.77703300$ H $5.20955800 \quad 0.90605200 \quad 0.37900000$ H $9.72717500-0.84278100-1.98243300$ H $4.51071100 \quad 0.417102001 .85704800$ Au $-0.29305800 \quad 1.22859000 \quad 0.17305000$ Au - $2.74205100 \quad-0.23508300 \quad-0.07846100$ Au $-2.60636300 \quad 2.48255400 \quad 0.08621400$ Au $-3.57566400 \quad-2.70291700 \quad-0.27901400$ $\mathrm{H}=-1208.193434$ Hartree $G=-1208.287984$ Hartree
\end{abstract}

REACTANTS, PRODUCTS, TRANSITION STATES

Cysteine- $\mathrm{H}_{2} \mathrm{O}+$ cysteine sulfenic acid C, $0,4.2545898186,-0.6043097116,-1.6300211389$ $\mathrm{H}, 0,4.9967004586,-1.2558070917,-2.1085493306$ S, $0,2.6363907074,-1.1576546909,-2.4465381364$ S, $0,-2.1227216608,1.5837767059,1.1197772645$ C, $0,-3.2438085116,0.14869983,1.6819706557$ $\mathrm{H}, 0,-3.7137374763,0.4363710916,2.6229037316$ $\mathrm{H}, 0,-2.6117859962,-0.7262940988,1.8562347672$ $\mathrm{H}, 0,4.4548775446,0.4284478541,-1.9196376123$ $\mathrm{H}, 0,-0.9184522931,0.7675977918,0.8324623852$ $0,0,1.4746378152,0.0635126504,-1.8102412038$ $\mathrm{H}, 0,1.5406586453,0.9061788288,-2.3168026926$ $0,0,0.2303298211,-0.1172444803,0.5286954915$ $\mathrm{H}, 0,1.0059135242,-0.1118376315,1.1530186759$ $\mathrm{H}, 0,0.5997935697,-0.055213045,-0.4003859491$ C, $0,4.3272444793,-0.7326904632,-0.1020691319$ $\mathrm{H}, 0,5.3716673818,-0.5646147244,0.182691916$ $\mathrm{N}, 0,3.9502825349,-2.1169760919,0.3877151365$ $\mathrm{H}, 0,3.4606841203,-1.9793263531,1.2937420692$ $\mathrm{H}, 0,3.2932981411,-2.5813217013,-0.256847037$ C, $0,3.4814507175,0.3125015858,0.6781059095$ $0,0,2.7214518559,-0.1576674797,1.6293696941$ $0,0,3.6254285775,1.5420736997,0.3539262643$ $\mathrm{H}, 0,4.7767264914,-2.7151904712,0.5078851863$ C, $0,-4.3445149516,-0.1836837019,0.6647765885$
C,0,-5.4253710242,0.9192594452,0.4903462578 O,0,-5.8840058253,1.471079017,1.5594035402 $0,0,-5.8133013788,1.1598657388,-0.7264864889$ $\mathrm{N}, 0,-3.7581494685,-0.4697487596,-0.7029799004$ $\mathrm{H}, 0,-2.9308692467,0.1348291741,-0.8478284289$ $\mathrm{H}, 0,-3.4884783208,-1.4540592384,-0.8122985101$ $\mathrm{H}, 0,-4.8630694876,-1.0924341068,0.9914995098$ $\mathrm{H}, 0,-4.4795205129,-0.1998245716,-1.396999183$ $\mathrm{H}=-819.040000$ Hartree

Cystine- $2 \mathrm{H}_{2} \mathrm{O}$ C $1.96452-0.8960 .28421$ H $1.67048-1.2275-0.7153$ S 0.62480 .352950 .86894 S - $0.590280 .49595-1.09081$ C - $1.98695-0.78266-0.87626$ H - $-1.54593-1.7465-0.61023$ H - $2.37504-0.84438-1.89921$ H $1.93869-1.73770 .97611$ H -1.60439 2.70014 - 0.79341 O 0.655183 .721370 .90691 H 1.041162 .828060 .80345 O -1.71503 $3.55497-0.31129$ H - 2.327013 .436660 .44311 H -0.19351 3.767640 .37063 C $3.35761-0.26310 .28271$ H 3.58880 .17951 .25671 C -3.12895 -0.374980 .05869$ H -3.42999 $0.6579-0.12773$ N -2.74304 -0.51806 1.52299 H -3.234730.17755 2.09876 H -3.07434 -1.46416 1.80477 O -5.02729-1.09737-1.26201 H -1.73198 -0.43361 1.6996 C - $4.35717-1.30972-0.18726$ O $-4.57491-2.221240 .70817$ C $4.46699-1.32854-0.0072$ O $4.43361-2.408190 .68639$ O $5.33927-0.99775-0.90795$ N $3.497290 .83598-0.7563$ H $3.521991 .76995-0.32831$ H $4.402740 .62982-1.23747$ H $2.737950 .81433-1.44942$ $\mathrm{H}=-819.056909$ Hartree 
TS

C 2.2806901361,-0.6808577193,-1.5181636019 H 1.6205976394,-1.2186092421,-2.2118874149 S 1.2210568505,0.8101552547,-0.9833641123 S - $1.7431567652,1.4198193817,-0.6639080057$ C - $1.9949634566,0.2935453665,0.8539897804$ H - 2.4578038785,0.872271587,1.6562012956 H - $1.0128545604,-0.0521427637,1.1960322321$ H 3.1378497009,-0.310389162,-2.0828973439 H -0.3045621219,2.7418429605,0.399067264 O 2.4767359231,2.0081301964,0.0185117847 H 3.0894023232,2.4884197877,-0.5847086212 O $0.5177431101,3.1408977251,0.8394932318$ H 0.4433033452,4.1008874603,1.0033558707 H 1.5865572045,2.6812238912,0.4884623792 C 2.7561147013,-1.5959268279,-0.3855159437 H 3.201152185,-2.484939385,-0.8467116213 N 1.6204441701,-2.0826605763,0.4933455987 H 2.0378195305,-2.2489767258,1.4298261045 H 0.8917337604,-1.3609261157,0.6027878229 C $3.8485488188,-0.9837640946,0.539079577$ O 3.7143026737,-1.2114642644,1.809440097 O $4.8136272621,-0.3577225058,-0.0350140589$ H 1.188545509,-2.9381167675,0.1230242034 C - $2.8929902935,-0.9153550659,0.5438917638$ C $-4.3877319976,-0.5664744703,0.3162539076$ O $-4.9487959072,0.2084885842,1.1795505128$ O $-4.970755613,-1.1278658861,-0.7010856606$ N - $2.392785384,-1.6385294126,-0.6923605929$ H - $1.9698719358,-0.9313951358,-1.3248288731$ H - $1.6977775386,-2.3587221109,-0.4645377791$ H - 2.8498320001,-1.6255257695,1.3778648189 H -3.2209933918,-2.048504194,-1.1602776157 $H=-819.011999$ Hartree

$\mathrm{TS}_{\mathrm{Ag} 2}$

C 2.6139323739,-0.0802511085,0.7416395759 H 2.3983427516,-1.1554649466,0.7952298422 S 1.0275687376, $0.6908116314,0.0136747274$ S - $1.3917303482,-0.2659214872,-0.9635031135$ C - $2.3789662956,-0.6601862372,0.6146766694$ H -1.6434816783,-0.7558442435,1.4195340254 H - $2.8712970459,-1.624053199,0.4604867192$ H 2.7192664684,0.3061873291,1.7566529765 H -1.2070192833,1.5717146192,-0.7843481766
O 1.3573992688,2.6679443382,0.2410584524 H 2.0344859512,2.9819753224,-0.4048347375 O $-0.9759054058,2.6617424175,-0.5724819025$ H -1.6753356811,3.0993178411,-0.0455573755 H 0.1177560309, $2.778732481,-0.1837359873$ C $3.8893953028,0.210887934,-0.0624173615$ H 4.0424547507,1.2879623061,-0.1592119216 C - $3.4120938842,0.4177379145,0.9997179959$ H - 2.9269138282,1.3724153843,1.2092677879 N -4.0937417935,-0.0452732809,2.2848019721 H -3.4134567045,-0.4183309954,2.9600676308 H - $4.6026305578,0.7327584219,2.7256859655$ O - $4.4609282339,1.6174939556,-0.8419000247$ H - $4.7766606713,-0.7779303975,2.0324568633$ C $-4.5306258764,0.5951608426,-0.0598965889$ O $-5.4488083933,-0.3227059572,-0.0690706715$ C 5.123889297,-0.4050084827,0.667066066 O $5.4480047543,0.1203550399,1.7943332673$ O 5.7024730963,-1.403673573,0.0742169203 N 3.8422869555,-0.3944118143,-1.4531682156 H 2.8948806945,-0.6663446024,-1.7438253733 H 4.2180152764,0.2594459087,-2.1513124954 H 4.4657448315,-1.2290818907,-1.4120098457 Ag -2.9782158094,-0.8917144386,-2.9946946254 Ag -4.3523765304,-1.6088260163,-5.1271979664 $\mathrm{H}=-1110.634896$ Hartree

$\mathrm{TS}_{\mathrm{Ag} 4}$

C $6.14841900-0.13840500-1.79659800$ H $5.59440300-0.70910100-2.55269900$ S $4.797876000 .82579200-0.86727300$ S $1.875858000 .50858600-0.07910400$ C $2.15969100-0.901412001 .18399200$ H $1.81545300-0.582576002 .16866300$ H $3.24011100-1.073015001 .22554300$ H $6.786034000 .57994600-2.31549000$ H 3.082350002 .005826001 .03951300 O 5.852094002 .162926000 .18930200 H $6.207832002 .88476300-0.37842800$ O 3.835797002 .550024001 .44596800 H 3.544321003 .411881001 .80131500 H 4.911350002 .473787000 .88543900 C $7.01088600-1.06637100-0.93306200$ H $7.63696200-1.65817400-1.60972500$ N $6.18529100-2.04527800-0.11863000$ H $6.66278400-2.102020000 .80607800$ 
H $5.22582000-1.702759000 .03691800$ C $7.97654600-0.333874000 .04380200$ O $7.99353800-0.769441001 .26717300$ O $8.707008000 .59979700-0.45265600$ H $6.14294100-2.97025300-0.56217500$ C $1.43694400-2.210801000 .80933300$ C $-0.09311400-2.126879000 .99949700$ O - $0.51827200-1.791278002 .16111300$ O $-0.84143400-2.40660700-0.03581000$ N $1.75228600-2.58696500-0.62517400$ H $1.76923400-1.72080400-1.19472800$ H $2.66800900-3.04891500-0.69080800$ H $1.81054200-3.018175001 .44801700$ H $1.01306100-3.20217500-0.99282700$ Ag -0.700722001 .227339000 .07868300$ Ag -3.38045200 $1.60754500-0.02224900$ $\mathrm{Ag}-2.78290600-1.07756300-0.13311700$ Ag $-5.44485800-0.49326800-0.28945500$ $H=-1402.204879$ Hartree

$\mathrm{TS}_{\mathrm{Au2}}$

C 1.1636392478,2.2256576486,1.2637637279 H 2.1231227733,2.5332046074,1.686082006 S $1.0916912309,0.2992485303,1.3898896079$ S 2.1254227246,-2.0669339275,-0.1430605426 C 3.9427616093,-1.5995755221,0.1793033811 H 4.4907752832,-2.5159278403,0.4269508395 H 3.988372974,-0.920575709,1.0342519569 H 0.3395468075, 2.5830891944, 1.8834482367 H 1.669313616,-2.3958860322,2.0114073001 O 0.6609129755,0.0188104274,3.3287923036 H - $0.301069489,0.1670914398,3.4953117165$ O 1.4548575809,-2.2561613858,2.9945243326 H 1.1207004938,-3.0486401426,3.4582566023 H 1.0418578679,-1.2489550556,3.2844815032 Au -3.373786571,-0.8260160746,-0.8418875537 $\mathrm{Au}-1.1260899107,-0.2457969019,0.261837461$ C 4.5853615568,-0.9010377041,-1.0363223203 H 4.0810876606,0.0483106099,-1.2298817918 N 4.4332061108,-1.7721878836,-2.267614174 H 4.4340759015,-1.2077119949,-3.1262333489 H 5.2466003867,-2.409628871,-2.3006116837 C 6.0941974602,-0.657972106,-0.7908650809 O $6.9249900693,-1.4757202695,-1.3614791851$ O $6.3983022509,0.3193107911,-0.0045460424$ H 3.5434292323,-2.2977288178,-2.2065724871 C $0.9639119156,2.7276715821,-0.1646986244$
C $0.6998734385,4.2724885362,-0.2028024111$ O $-0.1190727669,4.7478801857,0.663174072$ O 1.3093346176,4.9193532732,-1.1463014343 N 2.1577316798,2.4578148483,-1.0634971316 H 2.0358653748,1.6120625446,-1.6340549967 H 2.2293019758,3.3021022787,-1.6718544329 H 0.0924903408,2.2491240884,-0.626650339 H 3.0339085808,2.3734106524,-0.5297984669 $\mathrm{H}=-1089.987099$ Hartree

\section{STRUCTURES FOR REDOX POTENTIAL COMPARISON}

Thiophenol

C - $0.6708391272,1.0875562847,0.0108849934$ C $0.7376937827,1.0777432574,-0.0127472999$ C $1.442606509,2.2995153985,-0.0357426348$ C $0.7457272137,3.524879214,-0.0354355855$ C $-0.6637864443,3.5229283502,-0.0116963116$ C - $1.3772902697,2.3078762347,0.011650061$ H - $1.2095561262,0.1436417413,0.028627169$ H 1.2707491584,0.1304007418,-0.0132124766 H 1.2824515734,4.4703492339,-0.0532819117 H - $1.1968657393,4.4702270391,-0.0115943986$ H - 2.4638397269,2.3113212993,0.0300320081 S 3.2904936053,2.2215333251,-0.0658821246 H 3.5203442511,3.5786184999,-0.0840613082

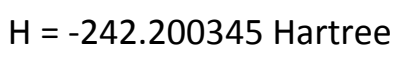

Homocysteine

S $4.7157252914,4.6869894724,1.6661905015$ H 4.5565617052,4.9722256388,3.0062502961 O 2.8849084164,0.2283859506,4.9992384846 O 4.5218068475,-0.1202934294,3.4095009609 N 2.2784592234,2.6467866196,4.2839633492 H 2.5015289223,3.5568509574,4.7032024202 H 1.4504182627, 2.7537535673,3.6836828025 H 2.0703204446,1.9412453733,5.0248444615 C 3.153018242,3.6075217234,1.4498094742 H 3.0104370831,3.5715664736,0.3664116575 H 2.3118963018,4.1552153407,1.883924629 C $3.6369546822,0.599844858,4.0040920804$ C $3.2779008245,2.1768979578,1.9971432291$ H 4.1288825683,1.6758007263,1.5258500567 H 2.3725473432,1.6235419094,1.7049126622 
C 3.4629931591,2.066956108,3.519530323 H 4.3527406823,2.6217107528,3.8346526115 $H=-372.992589$ Hartree

Penicillamine C 0.4662499162,2.391772376,9.3215877397

C - $0.0995783001,3.7272568236,8.7399598371$ C - $0.9081409199,3.6312754808,7.4104187664$ C - $1.3726941656,5.0344212987,6.9579016862$ C - $2.1295782298,2.703959839,7.5432043283$ O 1.7639536244,2.3019027426,9.3797198989 O - $0.3714225699,1.5140363648,9.74022467$ S 0.3175874369,2.8998626326,6.0702382869 N 1.0918128082,4.6670241405,8.5969289864 H - $0.7725105648,4.1550472518,9.4890923773$ H - $0.549854508,5.7310402268,6.7634661528$ H - $1.9630370477,4.9521440704,6.038128804$ H - $2.0153477305,5.4691054384,7.7354774908$ H - $2.7033484606,2.7236885551,6.6096217705$ H - 2.7802711752,3.0612039602,8.3535129674 H - $1.8443858956,1.6730165334,7.7589864579$ H - $0.3731079985,3.3820448967,4.9809810461$ H 0.9565300948,5.5386904826,9.1214474147 H 1.2875235535,4.8914887102,7.6116964397 H 1.9043201321,4.1281181755,8.9711048791 $H=-412.263901$ Hartree

Glutathione S 6.3368794398,7.9996723264,13.0301570687 O $0.1840433643,1.8594456231,12.5458766563$ O - $0.639402466,3.1355285592,10.8077834991$ O 2.1319700991,6.5986426286,9.8537370531 O $4.7917510151,10.3298403733,10.3244316936$ O 5.5224107951,10.8568398006,6.3487733575 O $3.8105043696,9.5257529068,7.1184956714$ N 2.5656125326,2.8993040082,12.5746411808 N 4.1584021586,6.7436639026,10.9538987494 N 5.3567738196,8.4501477529,9.1173006451 C $1.6138547723,3.5836870097,11.5994613452$ C $2.2111898015,3.6717282117,10.1842411531$ C $3.4703309671,4.5563769673,10.0763504482$ C $3.1899273744,6.0380041654,10.293834913$ C $0.2768507683,2.7930338055,11.6435993713$ C $4.0661997176,8.1792967372,11.2249046631$ C $4.5447102562,8.5238088087,12.6489310975$
C 4.775881896,9.0631061885,10.1764178283 C 6.0295919956,9.2024384854,8.0665556916 C 5.1143529865,9.9539700436,7.1092935166 H 6.9614902453,8.9581592978,12.2599782645 H 3.2474548021,10.0171501481,6.4742706086 H 3.0121819659,3.5697380661,13.2099237083 H 3.2983662703,2.3730411551,12.0817063601 H 1.9684741434,2.2293674425,13.1068076778 H 4.9716220566,6.2470338565,11.3120691644 H 5.2793016123,7.4425888293,9.0224247528 H 1.4163809191,4.5856855499,11.9937681529 H 1.4329377249,4.0636247622,9.5234907369 H 2.4571740807,2.6611262988,9.8331389231 H 3.8665642876,4.4570521542,9.0557823574 H 4.2633437103,4.2149933198,10.7521831248 H 3.0073368912,8.4625750906,11.1709156278 H 3.9387987918,7.988782666,13.3830620547 H 4.459857527,9.5963332332,12.8244792423 H 6.6238684671,8.508393679,7.4627681255 H 6.7177108413,9.9379661463,8.4934455151 $H=-1016.679759$ Hartree

Cysteine- $\mathrm{Ag}_{2}$ complex N 0.1397423746,1.674189629,1.5785080262 C $0.4030044257,0.4048796275,0.792462602$ H 1.3161013734,-0.0382524801,1.2065331191 C $-0.7359396448,-0.6057548863,0.9607026139$ C $0.7094463537,0.7864482188,-0.6795216717$ H - $0.4872569104,-1.5226153845,0.4269502866$ H - $0.8918170215,-0.8388819038,2.0172098363$ S - $2.4246268975,0.0172048425,0.3417879578$ O $1.0210757156,2.0369083722,-0.8839683421$ H - $2.1236329117,-0.0038357763,-1.0034180811$ O 0.6723036492,-0.1409754731,-1.5592776686 Ag 1.5055475248,2.8065229589,-3.0097597537 H 0.7311238381,2.4231633443,1.1832048405 H -0.8456486944,1.9629035285,1.4758754659 H 0.345873017,1.5426576763,2.5765895896 Ag 2.0758975984,3.821581826,-5.3707072008 $H=-625.302947$ Hartree

Cysteine- $\mathrm{Au}_{2}$ complex N 3.637600808,-1.5884696484,-0.8477201967 C 3.5434509819,-0.8766757061,0.4871413354 H 3.7070894771,-1.6348848396,1.2616662865 
C 4.63410369, $0.1914736281,0.6255170675$

C 2.1059229787,-0.3374340097,0.6767304829

H 4.5704808005,0.6491699215,1.6122894363

H 5.6244180213,-0.2541401855,0.5017276647

S 4.543234241,1.5636704341,-0.6899701575

O 1.2109064707,-0.8693099337,-0.1240731716

H 3.4042482077, 2.17564717,-0.2117310714

O $1.8892588827,0.5248077228,1.5858002065$

H 2.7417158451,-2.0665019414,-1.0305320484

H 3.7930806734,-0.9065352802,-1.606845584

H 4.4096155397,-2.2673195005,-0.8481439863

Au $-0.913120748,-0.3263463361,-0.0608328086$

$\mathrm{Au}-3.4303199798,0.2063991845,-0.0892998951$

$H=-604.693458$ Hartree

CYSTEINE (X-ray structure)

C - $1.4212143689,0.5084941488,10.3045833497$

O $-1.8030645635,0.8386234369,11.5011517273$

O $-0.2685272293,0.7252706301,9.7783430428$

C - $2.4615658201,-0.2719840628,9.4419461049$

H - $2.1558113748,-1.3227754417,9.4382476852$

N -3.7939486441,-0.1978471081,10.1706726436

H - $4.4033436682,0.5325308448,9.7792656986$

H -3.5546153793,0.0707829157,11.1485685901

H -4.2942680147,-1.0942912729,10.1524958799

C - $2.5487660475,0.2590074733,8.0097114945$

H - $1.5510789726,0.2448190377,7.5691318123$

H - $2.931748967,1.2825735252,7.982759881$

S -3.6044651587,-0.8095232976,6.8379861891

H -4.8414817912,-0.3522808294,7.242035901

$H=-333.711888$ Hartree 